\title{
Methodische Grundlagen der Züchtung von Saflor (Carthamus tinctorius L.) für den ökologischen Landbau
}

\author{
Dissertation \\ zur Erlangung des Doktorgrades \\ der Fakultät für Agrarwissenschaften \\ der Georg-August-Universität Göttingen \\ vorgelegt von \\ Sabine Rudolphi \\ geboren in Paderborn
}

Göttingen, im Mai 2007 
D7

1. Referent: Prof. Dr. H. C. Becker

2. Korreferentin: Prof. Dr. E. Pawelzik

Tag der mündlichen Prüfung: 24. Mai 2007 
Ohne Spekulation gibt es keine neue Beobachtung.

Charles Darwin (1809 - 1882) 


\section{Inhaltsverzeichnis}

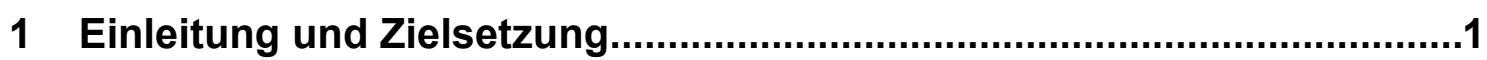

2 NIRS zur Bestimmung von Qualitätsmerkmalen bei Saflor.................... 4

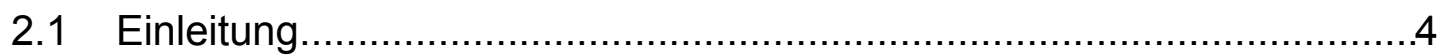

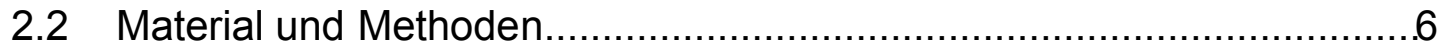

2.2.1 Spektrenaufnahme und Kalibrationsentwicklungen...................... 6

2.2.2 Kalibrationsentwicklung für Ölgehalt........................................

2.2.3 Kalibrationsentwicklung für Linolsäure- und Ölsäuregehalt............ 8

2.2.4 Kalibrationsentwicklung für Schalenanteil und Anteil tauber

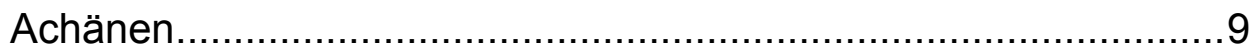

2.2.5 Merkmalskorrelationen..........................................................

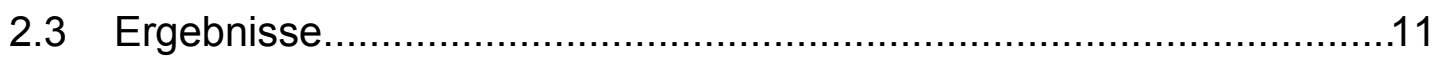

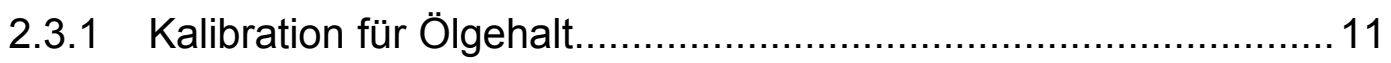

2.3.2 Kalibration für Linolsäure- und Ölsäuregehalt............................. 14

2.3.3 Kalibration für Schalenanteil und Anteil tauber Achänen..............16

2.3.4 Merkmalskorrelationen.........................................................18

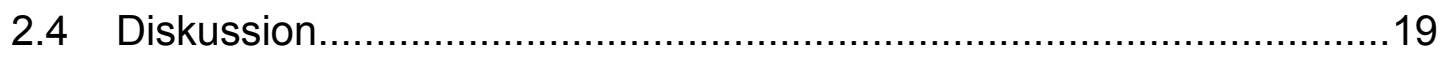

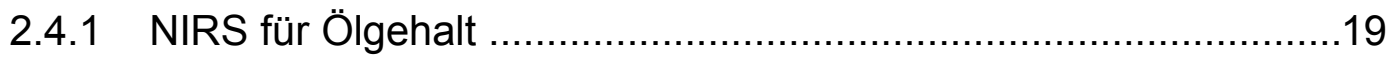

2.4.2 NIRS für Linolsäure- und Ölsäuregehalt....................................20

2.4.3 NIRS für Schalenanteil und Anteil tauber Achänen...................... 21

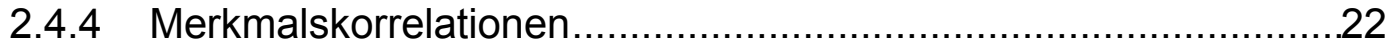

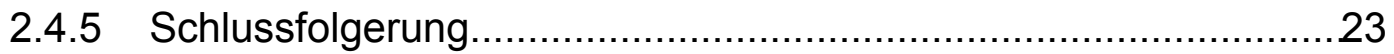

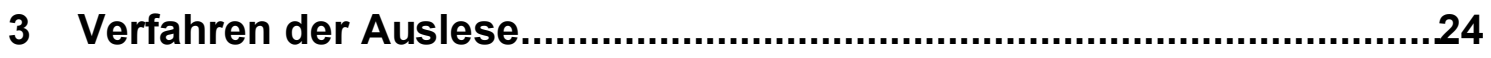

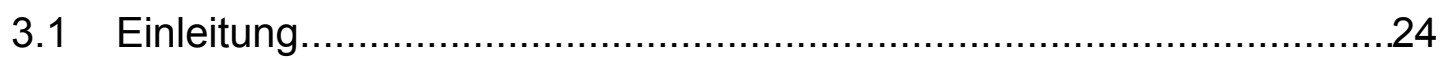

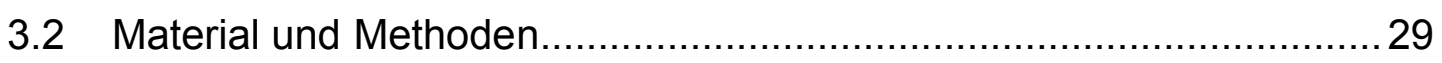

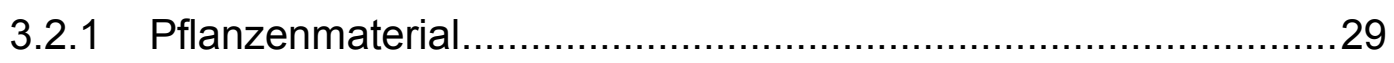

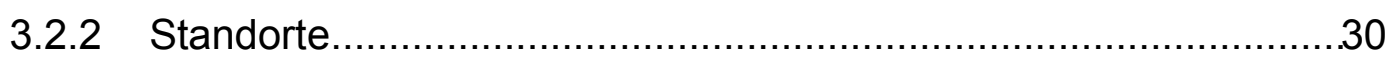

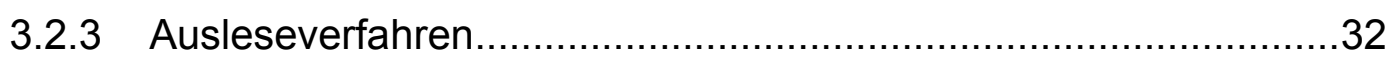

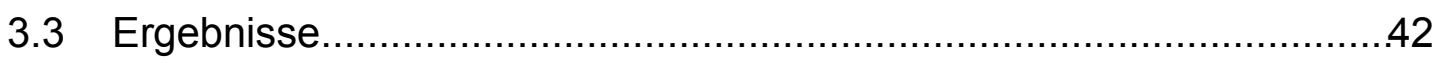

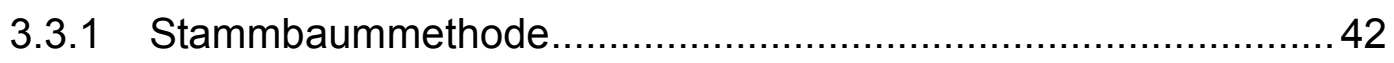

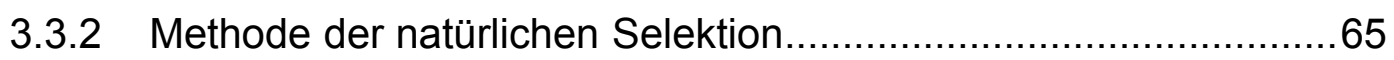

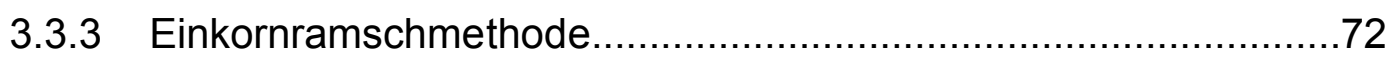

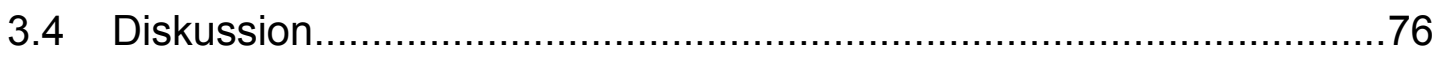

3.4.1 Bewertung der Stammbaummethode.......................................76 
3.4.2 Bewertung der Methode der natürlichen Selektion..................... 80

3.4.3 Bewertung der Einkornramschmethode.............................. 82

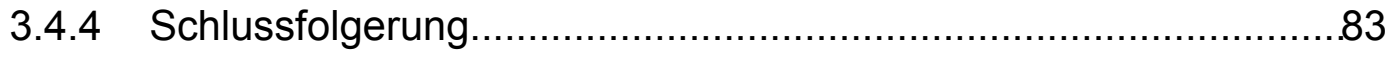

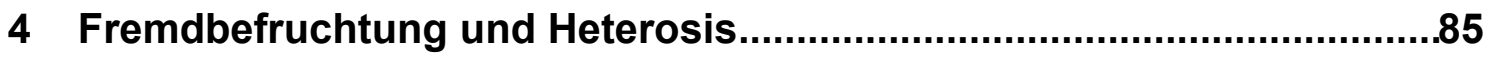

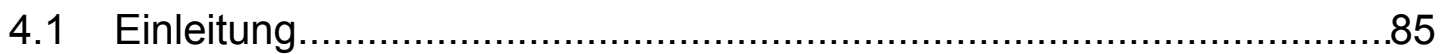

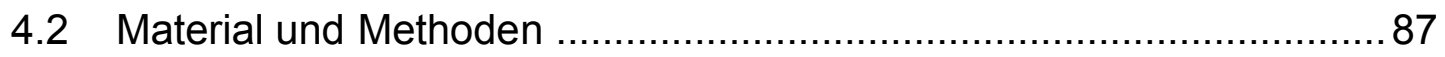

4.2.1 Untersuchungen zur Fremdbefruchtung..............................87

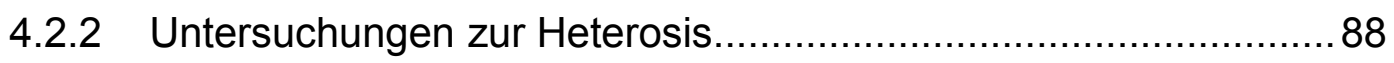

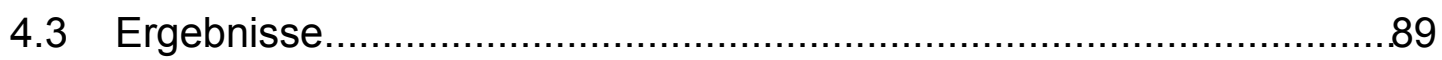

4.3.1 Untersuchungen zur Fremdbefruchtung................................ 89

4.3.2 Untersuchungen zur Heterosis.......................................90

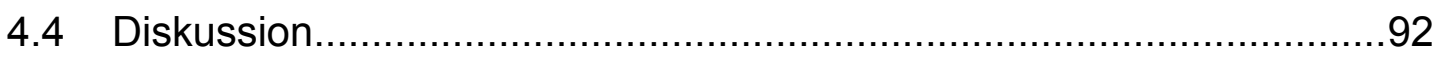

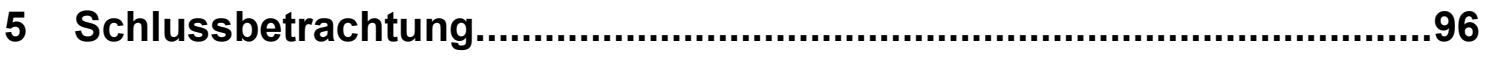

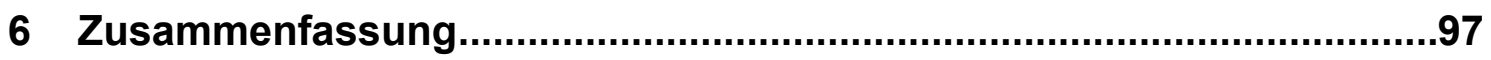

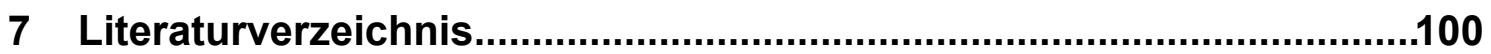

8 Anhang 


\section{Tabellenverzeichnis}

Tab. 1: Übersicht der Proben für die Kalibrationen zur

Ölgehaltsbestimmung.

Tab. 2: Übersicht der Proben für die Kalibrationen zur Linolsäure- und

Ölsäuregehaltsbestimmung....

Tab. 3: Statistik der Kalibrationen und der Kreuzvalidierungen für Ölgehalt (\%)....

Tab. 4: $\quad$ Statistik der Kalibrationen und der Kreuzvalidierungen für Linolsäure- und Ölsäuregehalt (\%)...

Tab. 5: Statistik der Kalibrationen und der Kreuzvalidierungen für Schalenanteil (\%) und Anteil tauber Achänen (\%)...

Tab. 6: Spearman's Rangkorrelationen zwischen den Merkmalen Ölgehalt, Linolsäure- und Ölsäuregehalt, Schalenanteil und Anteil tauber Achänen ( $\mathrm{N}=146)$.

Tab. 7: Durchschnittliche Monatstemperatur $\left({ }^{\circ} \mathrm{C}\right)$ über die Vegetationsperioden 2004 bis 2006 an den vier Standorten

Tab. 8: Summe der monatlichen Niederschläge $(\mathrm{mm})$ über die Vegetationsperioden 2004 bis 2006 an den vier Standorten

Tab. 9: Aussaatparameter, Erntetermin und Vorfrucht der Versuchsstandorte.

Tab. 10: Boniturergebnisse für das Merkmal Lager (Note) in Göttingen 2004

Tab. 11: Ergebnisse der Varianzanalyse für das Merkmal Köpfchenfäule (Note) an vier (Kreuzung 3: zwei) Standorten 2006.

Tab. 12: Köpfchenfäule (Note), Befall an den Versuchsstandorten und im Mittel der Orte.

Tab. 13: Ergebnisse der Varianzanalyse für das Merkmal AlternariaBlattflecken (Note) an zwei Orten 2006.

Tab. 14: Alternaria-Blattflecken (Note), Befall an den Versuchsstandorten und im Mittel der Orte.

Tab. 15: Ergebnisse der Varianzanalyse für das Merkmal Fusarium (\%) an vier (Kreuzung 3: zwei) Standorten 2006.

Tab. 16: Fusarium (\%), Befall an den Versuchsstandorten und im Mittel der Orte. 
Tab. 17: Ergebnisse der Varianzanalyse für das Merkmal Ölgehalt (\%) an vier (Kreuzung 3: zwei) Standorten 2006.

Tab. 18: Ölgehalt (\%), Daten der Versuchsstandorte und im Mittel der Orte.

Tab. 19: Ergebnisse der Varianzanalyse für das Merkmal EP-Ertrag (g) an vier (Kreuzung 3: zwei) Standorten 2006.

Tab. 20: EP-Ertrag (g), Daten der Versuchsstandorte und im Mittel der Orte

Tab. 21: Ergebnisse der Varianzanalyse für das Merkmal TKG (g) an vier (Kreuzung 3: zwei) Standorten 2006.

Tab. 22: TKG (g), Daten der Versuchsstandorte und im Mittel der Orte. 60

Tab. 23: Ergebnisse der Varianzanalyse für das Merkmal Schalenanteil (\%) an vier (Kreuzung 3: zwei) Standorten 2006.

Tab. 24: Schalenanteil (\%), Daten der Versuchsstandorte und im Mittel der Orte.

Tab. 25: Korrelationen der Merkmale in der Kreuzung 1.

Tab. 26: Korrelationen der Merkmale in der Kreuzung 2.

Tab. 27: Korrelationen der Merkmale in der Kreuzung 3.

Tab. 28: Keimfähigkeit (\%) des Erntegutes aus 2005

(bei R Ho Kreuzung 1 und Kreuzung 3 aus 2004).

Tab. 29: Feldaufgang (Pflanzen pro $\mathrm{m}^{2}$ ) an den vier Versuchsstandorten

Tab. 30: Ergebnisse der natürlichen Selektion der drei Herkunftsorte und der Kontrolle, im Mittel über die Standorte und Kreuzungen sowie die Varianzkomponenten

Tab. 31: Pflanzenlänge (cm) der Ramschparzellen 2006 aufgegliedert in Kreuzungen

Tab. 32: Pflanzenlänge (cm) der Ramschparzellen 2006 aufgegliedert in Standorte.

Tab. 33: Köpfchenfäule (Note) der Ramschparzellen 2006 aufgegliedert in Kreuzungen

Tab. 34: Köpfchenfäule (Note) der Ramschparzellen 2006 aufgegliedert in Standorte.

Tab. 35: Fusarium (\%) der Ramschparzellen 2006 aufgegliedert in Kreuzungen 
Tab. 36: Fusarium (\%) der Ramschparzellen 2006 aufgegliedert in Standorte 69

Tab. 37: Ölgehalt (\%) der Ramschparzellen 2006 aufgegliedert in Kreuzungen. .70

Tab. 38: Ölgehalt (\%) der Ramschparzellen 2006 aufgegliedert in Standorte .70

Tab. 39: EP-Ertrag (g) der Ramschparzellen 2006 aufgegliedert in Kreuzungen .70

Tab. 40: EP-Ertrag (g) der Ramschparzellen 2006 aufgegliedert in Standorte .71

Tab. 41: TKG (g) der Ramschparzellen 2006 aufgegliedert in Kreuzungen.

Tab. 42: TKG (g) der Ramschparzellen 2006 aufgegliedert in Standorte .71

Tab. 43: Merkmalsdaten der 50 Linien und zwei Eltern pro Kreuzung 74

Tab. 44: Fremdbefruchtung (\%) zwischen Parzellen 2004. 89

Tab. 45: Fremdbefruchtung (\%) innerhalb einer Parzelle 2004 und 2005. 89

Tab. 46: Ergebnisse der Untersuchungen zur Heterosis 91 


\section{Abbildungsverzeichnis}

Abb. 1: Küvetten für die NIRS-Messungen, links befüllt mit Mehl, rechts befüllt mit unvermahlenen Achänen

Abb. 2: Beziehung zwischen Referenzwerten und NIRS-Werten für Ölgehalt.

Abb. 3: Häufigkeitsverteilung der Referenzwerte (Soxhlet) für die Kalibrationen Ölgehalt 1, 2 und 3.

Abb. 4: Beziehung zwischen Referenzwerten und NIRS-Werten für Linolsäuregehalt (oben) und Ölsäuregehalt (unten).

Abb. 5: Beziehung zwischen Referenzwerten und NIRS-Werten für Schalenanteil (oben) und Anteil tauber Achänen (unten).

Abb. 6: Schema des Versuchsaufbaus

Abb. 7: Schema der Stammbaummethode.

Abb. 8: Köpfchenfäule; Mittlere Boniturnoten von Kreuzungen und Eltern (2004: 50 F3-Linien pro Kreuzung, 2005: 91, 97 und 72 F4-Linien pro Kreuzung)

Abb. 9: Alternaria-Blattflecken; Mittlere Boniturnoten von Kreuzungen und Eltern (2004: 50 F3-Linien pro Kreuzung, 2005: 91, 97 und 72 F4-Linien pro Kreuzung)

Abb. 10: Durchschnittlicher Ölgehalt (\%) 2004 und 2005 (2004: 35, 34 und 40 F3-Linien pro Kreuzung, 2005: 91, 97 und 72 F4-Linien pro Kreuzung)

Abb. 11: Köpfchenfäule, Boniturnoten der 29 (27) Linien, der Kontrolle (K) und der Eltern (E1, E2), gemittelt über vier (zwei) Versuchsstandorte.

Abb. 12: Alternaria-Blattflecken, Boniturnoten der 29 (27) Linien, der Kontrolle (K) und der Eltern (E1, E2), gemittelt über zwei Versuchsstandorte.

Abb. 13: Fusarium (\%) der 29 (27) Linien, der Kontrolle (K) und der Eltern (E1, E2), gemittelt über vier (zwei) Versuchsstandorte.

Abb. 14: Ölgehalt (\%) der 29 (27) Linien, der Kontrolle (K) und der Eltern (E1, E2), gemittelt über vier (zwei) Versuchsstandorte.

Abb. 15: EP-Ertrag (g) der 29 (27) Linien, der Kontrolle (K) und der Eltern (E1, E2), gemittelt über vier (zwei) Versuchsstandorte. 
Abb. 16: TKG (g) der 29 (27) Linien, der Kontrolle (K) und der Eltern (E1, E2), gemittelt über vier (zwei) Versuchsstandorte. .59

Abb. 17: Schalenanteil (\%) der 29 (27) Linien, der Kontrolle (K) und der Eltern (E1, E2), gemittelt über vier (zwei) Versuchsstandorte.....61

Abb. 18: Anzahl Linien pro Kreuzung und Merkmal besser als das Elternmittel $(A)$, als der bessere Elter $(B)$ oder als die Kontrolle $(\mathrm{C})$.

Abb. 19: Verteilung der Linien in den Ölgehaltsklassen (A),

TKG-Klassen (B) und EP-Ertragsklassen (C).

Abb. 20: Saflorblüten mit herausgeschobenen Griffeln und Pollen.

Abb. 21: Versuchsanlage zur Untersuchung der Fremdbefruchtung zwischen Parzellen 2004.

Abb. 22: Verteilung der Fremdbefruchtung (\%) 2004 und 2005 90 


\section{Einleitung und Zielsetzung}

Saflor (Carthamus tinctorius L.) gehört zu den ältesten Kulturpflanzen der Welt. Der Ursprung dieser distelartigen Pflanze wird im Orient angenommen. Vermutlich stammt Saflor von der Wildart Carthamus oxyacanthus Bieb., einem in Pakistan und Indien weit verbreiteten Unkraut, oder von Carthamus persicus Willd., einem Unkraut, das in Syrien, im Libanon und in der Türkei vorkommt, ab. Saflor ist diploid, besitzt $n=12$ Chromosomen und gehört zur Familie der Kompositen. Es handelt sich um eine einjährige, 30-150 cm große Pflanze mit gelben, orangenen oder roten Blüten. Sie bildet eine Pfahlwurzel aus und ist besonders für trockene Standorte gut geeignet (Knowles 1976, Dajue und Mündel 1996). Beste Erträge werden auf gut drainierten, sandigen Lehmböden mit guter Wasserkapazität in den tieferen Bodenschichten erzielt (Mündel et al. 2004). Saflor wird als Selbstbefruchter mit einer Auskreuzungsrate unter $10 \%$ beschrieben, wobei je nach Umweltbedingungen auch bis zu 50\% Fremdbefruchtung möglich sind (Knowles 1969, Dajue und Mündel 1996). Hauptsächlich findet die Fremdbestäubung durch Insekten statt, der Wind spielt fast keine Rolle (Knowles 1989).

Ursprünglich ist Saflor, auch Färberdistel oder falscher Safran genannt, zum Färben von Stoffen und Lebensmitteln angebaut worden. Die Blüten enthalten Carthamin, einen wasserunlöslichen roten Farbstoff und das wasserlösliche Carthamidin. Spuren von Saflor wurden in 4000 Jahre alten, ägyptischen Mumien gefunden (Weiss 1971). Im 18. Jahrhundert wurde der Saflor auch in Italien, Frankreich und Großbritannien zum Färben von Speisen genutzt (Dajue und Mündel 1996). Der Färberdistelanbau ging jedoch mit der Erfindung der Anilinfarben am Anfang des 20. Jahrhunderts zurück. In den 1930er Jahren wurde die Saflorpflanze in Deutschland als Lieferant von Öl wieder neu entdeckt (Lennerts 1984). Momentan wird hier jedoch fast kein Saflor mehr angepflanzt (Reinbrecht et al. 2003).

In der Gegenwart wird Saflor hauptsächlich für die Ölgewinnung genutzt. Der Ölgehalt variiert zwischen $20 \%$ und 45\% (Dajue und Mündel 1996). Das ernährungsphysiologisch wertvolle Öl enthält bis zu 88,8\% der ungesättigten Fettsäure Linolsäure (Fernández-Martínez et al. 1993) und zählt damit zu den linolsäurereichsten Ölen (Lennerts 1984). Linolsäure gehört zu den essenziellen Fettsäuren und kann nicht vom Menschen gebildet werden 
(Carvalho et al. 2006). Daher hat die Verwendung als Speiseöl die größte Bedeutung. Daneben spielen technische Anwendungen von Hochölsäuretypen, die einen Ölsäuregehalt bis 90,6\% erreichen (Fernández-Martínez et al. 1993), nur eine untergeordnete Rolle (Geisler 1991).

Die Hauptanbaugebiete sind heute Indien (210 000 t), Mexiko (212 $800 \mathrm{t}$ ), USA (87 $300 \mathrm{t}$ ), Argentinien (51 $000 \mathrm{t}$ ), Australien (10 $000 \mathrm{t}$ ) und Äthiopien (6 $000 \mathrm{t}$ ). Geringer Anbau findet in Ungarn (288 t) und Spanien (100 t) statt (http://www.fao.org). Die Weltproduktion schwankte im Zeitraum zwischen 1960 und 1990 zwischen 500000 und 1000000 Tonnen (Weiss 2000), im Jahr 2005 wurden etwa 717800 t produziert. Der Ertrag beträgt zwischen $16 \mathrm{dt} / \mathrm{ha}$ und $22 \mathrm{dt} / \mathrm{ha}$ (Mündel et al. 2004).

Nur auf etwa $2 \%$ der ökologisch bewirtschafteten Fläche werden in Deutschland Ölpflanzen kultiviert (SÖL 2003). Es existiert eine Nachfrage nach ökologisch erzeugtem Pflanzenöl. Der Färberdistelanbau ist auch unter den eher humiden Klimabedingungen Deutschlands möglich (Reinbrecht et al. 2003). Die Einführung von Saflor als neue Ölpflanze für den ökologischen Landbau könnte das Kulturpflanzenspektrum und die Fruchtfolge erweitern. Bisher wurde in Deutschland jedoch fast keine Saflorzüchtung durchgeführt, allerdings beschäftigte sich Scheibe mit der Züchtung von Saflor in den 1930er Jahren (Scheibe und Yekta 1934, Scheibe 1939). Die Untersuchungen wurden später jedoch nicht weiter verfolgt. Der niedrige Ölgehalt und die hohe Krankheitsanfälligkeit der Pflanze stellen ein vorrangiges Problem dar.

Das Ziel der vorliegenden Arbeit war es daher, verschiedene Züchtungsmethoden bei Saflor zu untersuchen und zu bewerten. Hierfür wurden die Stammbaummethode, die natürliche Selektion (Ramschmethode) und die Einkornramschmethode (ohne Selektion) gewählt. Diese werden im Kapitel 3.1 genauer erläutert. Die wichtigsten Zuchtziele waren ein höherer Ölgehalt und niedrigere Krankheitsanfälligkeit. In zwei Versuchsjahren wurden Fremdbefruchtung und Heterosis bei Saflor untersucht, da in der Literatur eine große Variation in der Fremdbefruchtungsrate beschrieben wird (Dajue und Mündel 1996).

Um Qualitätsmerkmale wie den Ölgehalt, das Fettsäuremuster und den Schalenanteil züchterisch zu verbessern, ist eine Methode erforderlich, mit der eine große Anzahl von Genotypen einfach und kostengünstig analysiert werden 
kann. Die Nah-Infrarot-Reflexions Spektroskopie (NIRS) ist bei vielen Kulturarten dazu geeignet, mehrere Qualitätsmerkmale in nur einer Messung zu erfassen. Studien bei Saflor mit NIRS sind hier nicht bekannt. Daher war es auch ein Ziel der vorliegenden Arbeit, diese Methode für Saflor zu modifizieren und anzuwenden.

Die Arbeit ist in drei Teile gegliedert. Zunächst folgt ein Kapitel, in dem die NIRS-Methode und die Entwicklung der NIRS-Kalibrationen genauer beschrieben und diskutiert werden. Anschließend werden die drei Ausleseverfahren behandelt und im vierten Kapitel die Fremdbefruchtung und Heterosis untersucht. 


\section{NIRS zur Bestimmung von Qualitätsmerkmalen bei Saflor}

\subsection{Einleitung}

Die Nah-Infrarot-Reflexions Spektroskopie ist eine kostengünstige, schnelle und präzise Methode zur Schätzung unterschiedlicher Qualitätskomponenten (Font et al. 2006, Baye und Becker 2004). Das Licht im nahen Infrarotbereich wird von Molekülbindungen absorbiert. Die Menge an absorbierter Energie ist ein Maß für die Menge an Molekülen, die absorbieren. Zunächst werden sowohl die NIRS-Spektren als auch die chemisch analysierten Referenzwerte für ein Qualitätsmerkmal von einem Probensatz aufgenommen. Mit diesen Informationen wird eine Gleichung für das Merkmal entwickelt, mit der die Merkmalswerte in nachfolgenden Proben anhand der Spektren ermittelt werden können (Stuth et al. 2003).

Zahlreiche Studien an verschiedenen Kulturarten wie Raps (Velasco et al. 1998, Míka et al. 2003, Hom et al. 2007), Sojabohne (Mündel und Schaalje 1988, Pazdernik et al. 1997), Vernonia (Baye und Becker 2004) und Sonnenblume (Pérez-Vich et al. 1998, Fassio und Cozzolino 2004, Moschner und Biskupek-Korell 2006) haben gezeigt, dass NIRS eine geeignete Methode darstellt, um Parameter wie Öl- und Proteingehalte, Fettsäure- und Aminosäurezusammensetzungen an Mehl, intakten Samenproben und sogar an Einzelsamen zu bestimmen. In Zuchtprogrammen, bei denen eine große Anzahl von Proben analysiert werden muss, können mehrere Qualitätskomponenten mit nur einer Messung geschätzt werden, ohne dass die oft geringe Menge an Saatgut zerstört werden muss (Font et al. 2006).

Bei Saflor sind bislang keine Untersuchungen mit NIRS bekannt. Der Ölgehalt ist eines der wichtigsten Zuchtziele bei Saflor, weil das Öl aufgrund seines hohen Gehaltes an Linolsäure als hochwertiges Speiseöl eingesetzt wird. Bei der Entwicklung von Linien mit verbessertem Ölgehalt und interessantem Fettsäuremuster fehlte bisher noch die Möglichkeit diese Parameter schnell und kostengünstig bestimmen zu können. Die Analyse von kleinen Saatgutmengen, wie sie bei der Einzelpflanzenernte anfallen, erwies sich als schwierig. Laboranalysen wie Soxhlet und Gaschromatographie sind zeitaufwändig und können meist nur an Mehlproben vorgenommen werden. 
Auch die Bestimmung des Schalenanteils, eines Merkmals, das eng mit dem Ölgehalt korreliert ist (Knowles 1969), erwies sich als sehr aufwändig. Bereits bei Scheibe (1939) wurde die Verringerung des Schalenanteils als ein wichtiges Zuchtziel beschrieben. Bei Saflor ist stets ein gewisser Prozentsatz tauber Achänen festzustellen, der sich auf die Aufgangsrate auswirkt (Scheibe 1939).

Das Ziel des ersten Teils der vorliegenden Arbeit war es, NIRS-Kalibrationen zunächst an Mehl und wenn möglich auch an intakten Achänen für den Ölgehalt, den Linolsäure- und Ölsäuregehalt, den Schalenanteil und den Anteil tauber Achänen zu entwickeln. 


\subsection{Material und Methoden}

\subsubsection{Spektrenaufnahme und Kalibrationsentwicklungen}

Zunächst wurden ca. $3 \mathrm{~g}$ intakter Achänen in einer Küvette mit einem Durchmesser von 4,7 cm mit einem Spektrometer des Typs NIRSystems 6500 (NIRSystems, Inc., Silver spring, MD) gemessen. Jede Probe wurde dreimal gemessen, wobei dieselbe Probenmenge bei jeder Messung neu in die Küvette eingefüllt wurde. Anschließend wurden diese $3 \mathrm{~g}$ Probenmaterial mit einer Mühle der Firma Retsch (Typ ZM100) auf eine Partikelgröße von 0,5 mm vermahlen und ebenfalls in einer runden Küvette einmal gemessen (Abb. 1). Es wurden die Absorptionsspektren (log 1/R) in 2-nm-Intervallen im Wellenlängenbereich von 400-2500 nm aufgenommen.

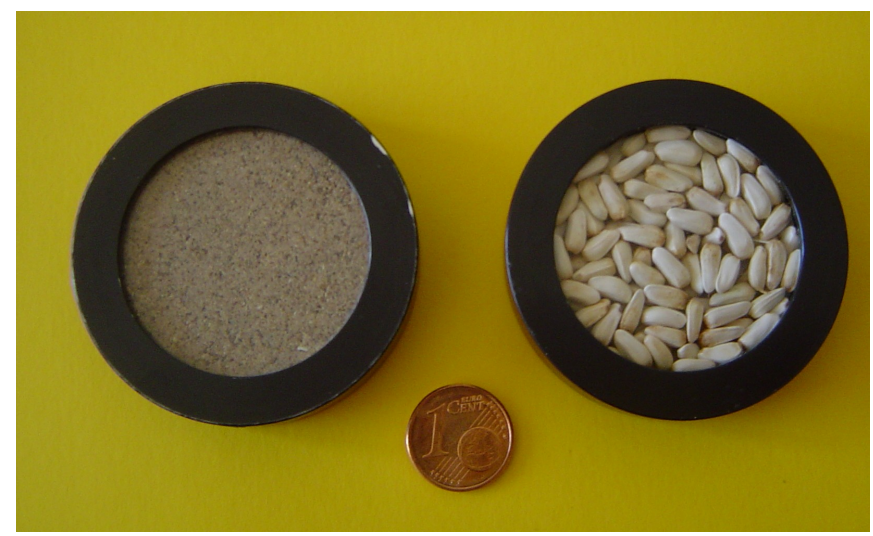

Abb. 1: Küvetten für die NIRS-Messungen, links befüllt mit Mehl, rechts befüllt mit unvermahlenen Achänen

Die Berechnungen erfolgten mit der Software WinISI II (Infrasoft International LLC, Analysis, calibration and network computer software Version 1.06) mit den Parametern: MPLS (modified partial least squares), math treatment 1,4,4,1, SNV/detrent. Die je drei Spektren der intakten Achänen wurden zunächst gemittelt und anschließend den jeweiligen Referenzwerten für Ölgehalt, Linolsäure- und Ölsäuregehalt, Schalenanteil und Anteil tauber Achänen zugeordnet. Den Spektren der Mehlmessungen wurden dieselben Referenzwerte zugeteilt.

Die Genauigkeit der Kalibrationen wurde anhand der Bestimmtheitsmaße der Kalibration und der Kreuzvalidierung beurteilt. Nach Fontaine et al. (2001) wurde der Wert SD/SECV (SD: Standardabweichung der Referenzmessung, 
SECV: Standardfehler der Kreuzvalidierung) hinzugezogen, um Kalibrationen für verschiedene Merkmale zu vergleichen.

\subsubsection{Kalibrationsentwicklung für Ölgehalt}

\section{Probenmaterial}

Um den Ölgehalt anhand gemahlener und ungemahlener Achänen zu schätzen, wurde 2004 je eine Kalibration entwickelt (Kalibration Ölgehalt 1) und in den Jahren 2005 (Kalibration Ölgehalt 2) und 2006 (Kalibration Ölgehalt 3) erweitert. Die Kalibrationen für intakte Achänen wurden für die Selektion verschiedener Genotypen eingesetzt (vergleiche Kapitel 3).

Für die Kalibrationen Ölgehalt 1 wurde zunächst das Erntegut von 1060 Einzelpflanzen aus Göttingen und das Erntegut von je drei Parzellen aus Göttingen, Hohenheim und Wilmersdorf mit dem NIRS-Gerät gemessen. Anhand der Spektren wurden mit der WinISI-Software 108 Proben so ausgewählt, dass eine möglichst große Variation entstand (Shenk und Westerhaus 1991). In 2005 und 2006 wurde der Ölgehalt von 300 und 950 Proben mit Hilfe der jeweils schon vorhandenen Kalibration geschätzt. Anhand dieser Werte wurden Proben herausgesucht, die sich besonders gut im Ölgehalt unterschieden und von verschiedenen Orten stammten (in 2005: Göttingen, Hohenheim und Wilmersdorf, 2006: zusätzlich Darzau). Außerdem wurden die Kalibrationen 2005 durch Proben aus Anbauversuchen 2002 und 2003 aus Göttingen und Hohenheim ergänzt.

In die Kalibrationsgleichungen wurden insgesamt 108 (Ölgehalt 1), 463 (Ölgehalt 2) und 571 (Ölgehalt 3) Proben einbezogen. Die Übersicht der Proben aus den verschiedenen Jahren und Herkunftsorten ist in der Tab. 1 dargestellt. 
Tab. 1: Übersicht der Proben für die Kalibrationen zur Ölgehaltsbestimmung

\begin{tabular}{|c|c|c|c|c|c|c|}
\hline \multirow[b]{2}{*}{ Kalibration } & \multirow[b]{2}{*}{ Herkunft der Proben } & \multicolumn{5}{|c|}{ Erntejahr } \\
\hline & & 2002 & 2003 & 2004 & 2005 & 2006 \\
\hline \multirow[t]{3}{*}{ Ölgehalt 1} & Göttingen & & & 102 & & \\
\hline & Hohenheim & & & 3 & & \\
\hline & Wilmersdorf & & & 3 & & \\
\hline \multirow[t]{3}{*}{ Ölgehalt 2} & Göttingen & 8 & 49 & 210 & 117 & \\
\hline & Hohenheim & & 46 & 30 & & \\
\hline & Wilmersdorf & & & 3 & & \\
\hline \multirow[t]{4}{*}{ Ölgehalt 3} & Göttingen & 8 & 49 & 210 & 117 & 27 \\
\hline & Hohenheim & & 46 & 30 & & 27 \\
\hline & Wilmersdorf & & & 3 & & 27 \\
\hline & Darzau & & & & & 27 \\
\hline
\end{tabular}

\section{Referenzanalytik}

Der Ölgehalt wurde mit der "Mini-Soxhletmethode“ analysiert, da nur sehr kleine Saatgutmengen zur Verfügung standen. Es wurden $500 \mathrm{mg} \mathrm{Mehl}$ in eine Extraktionshülse eingewogen, 15 Stunden bei $60^{\circ} \mathrm{C}$ getrocknet und anschließend erneut gewogen. Die Ölextraktion erfolgte mit Petrolether in einer Soxhletapparatur für 10 Stunden bei $70^{\circ} \mathrm{C}$. Nach erneutem Trocknen wurden die Proben wiederum gewogen und aus der Differenz der Gewichte vor und nach der Extraktion der Ölgehalt berechnet.

\subsubsection{Kalibrationsentwicklung für Linolsäure- und Ölsäuregehalt}

\section{Probenmaterial}

Mit Hilfe der WinISI-Software wurden die Proben ausgewählt, die eine größtmögliche Variation in den Spektren zeigten. Insgesamt wurden die Kalibrationen für intakte Achänen und Mehl auf der Grundlage von 534 Proben aus vier Jahren und von vier Standorten entwickelt (vergleiche Tab. 2). 
Tab. 2: Übersicht der Proben für die Kalibrationen zur Linolsäure- und Ölsäuregehaltsbestimmung

\begin{tabular}{|c|c|c|c|c|c|}
\hline \multirow[b]{2}{*}{ Kalibration } & \multirow[b]{2}{*}{ Herkunft der Proben } & \multicolumn{4}{|c|}{ Erntejahre } \\
\hline & & 2003 & 2004 & 2005 & 2006 \\
\hline Linolsäure- und & Göttingen & & 197 & 78 & 24 \\
\hline \multirow[t]{3}{*}{ Ölsäuregehalt } & Hohenheim & 121 & 30 & & 27 \\
\hline & Wilmersdorf & & 3 & & 27 \\
\hline & Darzau & & & & 27 \\
\hline
\end{tabular}

\section{Referenzanalytik}

Der Linolsäure- und Ölsäuregehalt wurde mit der GC-Analyse (Gaschromatographie) in Anlehnung an Thies (1971) bestimmt. Zu $200 \mathrm{mg}$ Mehl erfolgte eine Zugabe von $1 \mathrm{ml}$ 0,5 mol Na-Methylat. Nach dem Mischen wurde die Probe für 10 Minuten in ein $20^{\circ} \mathrm{C}$ warmes Wasserbad gestellt, wiederum gemischt und erneut für 10 Minuten in das Wasserbad gegeben. Nach der anschließenden Zugabe von $300 \mu \mathrm{l} 5 \% \mathrm{NaHSO} 4$ und $300 \mu \mathrm{l}$ IsoOctan wurde die Probe für 10 Minuten bei 2000 U/min zentrifugiert. $200 \mu \mathrm{l} \mathrm{der}$ oberen Phase wurden abpipettiert und im Gaschromatograf Model 8600 (Perkin Elmer Corp., Norwalk, CT, Kapillarchromatograph mit Autosampler AS 8300, Säule: FFAP, $25 \mathrm{~m} \times 0,25 \mathrm{~mm} \times 0,25 \mu \mathrm{m}, 200{ }^{\circ} \mathrm{C}$ isothermal, Trägergas: 120 $\mathrm{kPa} \mathrm{H} 2$ ) analysiert.

\subsubsection{Kalibrationsentwicklung für Schalenanteil und Anteil tauber Achänen}

\section{Probenmaterial}

Für die Entwicklung der Kalibrationen wurden Proben mit einer großen Variation im Ölgehalt aus Göttingen ausgewählt, da ein Zusammenhang zwischen dem Ölgehalt, dem Schalenanteil und dem Anteil tauber Achänen anzunehmen war (siehe Kapitel 2.2.5). Die Kalibrationen für intakte Samen und Mehl beruhten auf 40 Proben aus dem Jahr 2004, 10 Proben aus dem Jahr 2005 und 108 Proben aus dem Jahr 2006, also insgesamt auf 158 Proben.

\section{Referenzanalytik}

Je 20 Achänen wurden bei $60^{\circ} \mathrm{C}$ fünf Stunden getrocknet, gewogen und für 15 Stunden in Wasser eingeweicht. Anschließend wurden die Schalen abgetrennt und fünf Stunden bei $60{ }^{\circ} \mathrm{C}$ rückgetrocknet. Aus der Differenz zwischen dem 
Gewicht der gesamten Achänen und dem der Schalen ergab sich der Schalenanteil in Prozent. Die Anzahl tauber Achänen wurde in Prozent zu der Gesamtzahl untersuchter Achänen angegeben.

\subsubsection{Merkmalskorrelationen}

Von 146 Proben wurden die Korrelationen zwischen den Merkmalen Ölgehalt, Linolsäure- und Ölsäuregehalt, Schalenanteil und Anteil tauber Achänen untersucht.

Die Berechnung der Spearman's Rangkorrelationskoeffizienten erfolgte mit dem Programm PLABSTAT (PLAnt Breeding STATistical program, Version 2N(L),1997) (Utz 2001). 


\subsection{Ergebnisse}

Die Kalibrationsgleichungen werden im Folgenden sowohl für die intakten als auch für die gemahlenen Achänen (Mehl) dargestellt.

\subsubsection{Kalibration für Ölgehalt}

In Tab. 3 sind die Ergebnisse von sechs Kalibrationen dargestellt, je drei für die Schätzung des Ölgehaltes an intakten und gemahlenen Achänen. Bei den Kalibrationen Ölgehalt 2 und Ölgehalt 3 handelt es sich um eine jeweilige Erweiterung der vorangegangenen Kalibration.

Die Spannweite der Referenzwerte für die Kalibration Ölgehalt 1 betrug 10,03\% bis $29,57 \%$ mit einer Standardabweichung (SD) von 3,61. In 2005 wurde diese Kalibration unter anderem durch Proben mit sehr niedrigem (Minimum=3,26\%) und hohem (Maximum=30,63\%) Ölgehalt ergänzt $(S D=5,28)$. Bei den Proben handelte es sich um Erntegut aus den Jahren 2002, 2003 und 2005 aus Göttingen und Hohenheim. Aus der Einbeziehung weiterer Proben aus 2006 von vier Standorten resultierte eine Spannweite in den Referenzwerten von 3,26\% bis 32,39\% mit einer Standardabweichung von 5,30 (Kalibration Ölgehalt 3).

Die beste Übereinstimmung zwischen Referenzwerten und den NIRS-Werten wurde mit den Kalibrationen Ölgehalt 1 erreicht. Die Bestimmtheitsmaße der Kalibrationen für intakte $\left(R^{2}=0,91\right)$ und gemahlene Achänen $\left(R^{2}=0,92\right)$ differierten nur unwesentlich. Das Bestimmtheitsmaß der Kreuzvalidierung für die Variante intakte Achänen $(1-\mathrm{VR}=0,81)$ war dagegen niedriger als das der Variante Mehl (1-VR=0,91). Die Standardfehler waren bei der Kalibration und besonders bei der Kreuzvalidierung niedriger, wenn die Proben als Mehl gemessen wurden $(\mathrm{SEC}=1,01, \mathrm{SECV}=1,09)$.

Das Bestimmtheitsmaß der Kreuzvalidierung der Variante intakte Achänen in Ölgehalt 2 war etwas höher als das in Ölgehalt 1. Ansonsten zeigten die Kalibrationen Ölgehalt 2 in beiden Varianten höhere Standardfehler als die Kalibrationen Ölgehalt 1.

Das Bestimmtheitsmaß war in der Kalibration Ölgehalt 3 für die Messung intakter Achänen etwas geringer $\left(R^{2}=0,87\right)$, für die Kreuzvalidierung dagegen etwas höher $(1-V R=0,84)$ als in der Kalibrierung Ölgehalt 1. Für die Messung gemahlener Achänen waren die Bestimmtheitsmaße für die Kalibration $\left(R^{2}=0,91\right)$ und für die Kreuzvalidierung $(1-V R=0,87)$ geringer. 
Die Standardfehler der Kalibrationen und Kreuzvalidierungen Ölgehalt 3 waren, sowohl für die Messungen intakter als auch gemahlener Achänen, größer als in den Kalibrationen Ölgehalt 1.

Die erweiterten Kalibrationen zeigten allgemein etwas höhere Standardfehler, beruhten jedoch auf einem Probensatz mit größerer Variation im Ölgehalt und in den Herkunftsorten. Der Wert SD/SECV war bei der Messung intakter Achänen in den Kalibrationen Ölgehalt 2 und 3 höher als bei der Kalibration Ölgehalt 1. Bei der Variante Mehl war dies nicht der Fall.

Tab. 3: Statistik der Kalibrationen und der Kreuzvalidierungen für Ölgehalt (\%)

\begin{tabular}{|c|c|c|c|c|c|c|c|c|c|c|}
\hline \multirow[b]{2}{*}{ Merkmal } & \multicolumn{7}{|c|}{ Kalibration $^{\text {a }}$} & \multicolumn{2}{|c|}{$\begin{array}{c}\text { Kreuz- } \\
\text { validierung }\end{array}$} & \multirow[t]{2}{*}{$\begin{array}{l}\text { SDI } \\
\text { SECV }\end{array}$} \\
\hline & $\mathbf{N}$ & Mittel & SD & Min. & Max. & SEC & $\mathbf{R}^{2}$ & SECV & 1-VR & \\
\hline \multicolumn{11}{|c|}{ Intakte Achänen } \\
\hline Ölgehalt 1 & 108 & 20,41 & 3,61 & 10,03 & 29,57 & 1,09 & 0,91 &, 58 & 0,81 & 2,28 \\
\hline Ölger & 463 & 20,29 & 5,28 & 3 & 30 & 1,97 & 0,86 & 8 & 0,83 & 2,43 \\
\hline Ölgehalt 3 & 571 & 21,00 & 5,30 & 3,26 & 32,49 & 1,92 & 0,87 & 2,13 & 0,84 & 2,48 \\
\hline \multicolumn{11}{|l|}{ Mehl } \\
\hline Ölgehalt 1 & 108 & 20,41 & 3,61 & 10,03 & 29,57 & 1,01 & 0,92 & 9 & 0,91 & 3,33 \\
\hline Ölgehalt 2 & 463 & 20,29 & 5,28 & 3,26 & 30,63 & 1,46 & 0,92 & 1,75 & 0,89 & 3,03 \\
\hline Ölgehalt 3 & 571 & 21,00 & 5,30 & 3,26 & 32,49 & 1,59 & 0,91 & 1,88 & 0,87 & 2,81 \\
\hline
\end{tabular}

${ }^{a} \mathrm{~N}=$ Anzahl Proben, $\mathrm{SD}=$ Standardabweichung, SEC=Standardfehler der Kalibration, $\mathrm{R}^{2}=$ Bestimmtheitsmaß der Kalibration, ${ }^{\mathrm{b}}$ SECV=Standardfehler der Kreuzvalidierung, 1-VR=Bestimmtheitsmaß der Kreuzvalidierung

Die Abb. 2 zeigt die Ergebnisse der Kalibrationen Ölgehalt 1,2 und 3 in graphischer Form. Deutlich ist zu erkennen, dass der Probensatz der Kalibrationen Ölgehalt 3 eine größere Variation aufweist, die Bestimmtheitsmaße jedoch in den Kalibrationen Ölgehalt 1 höher waren. Bei den Kalibrationen Ölgehalt 2 und 3 stimmten bei einzelnen Proben die Referenzwerte nicht genau mit den Werten der NIRS-Messungen überein. 

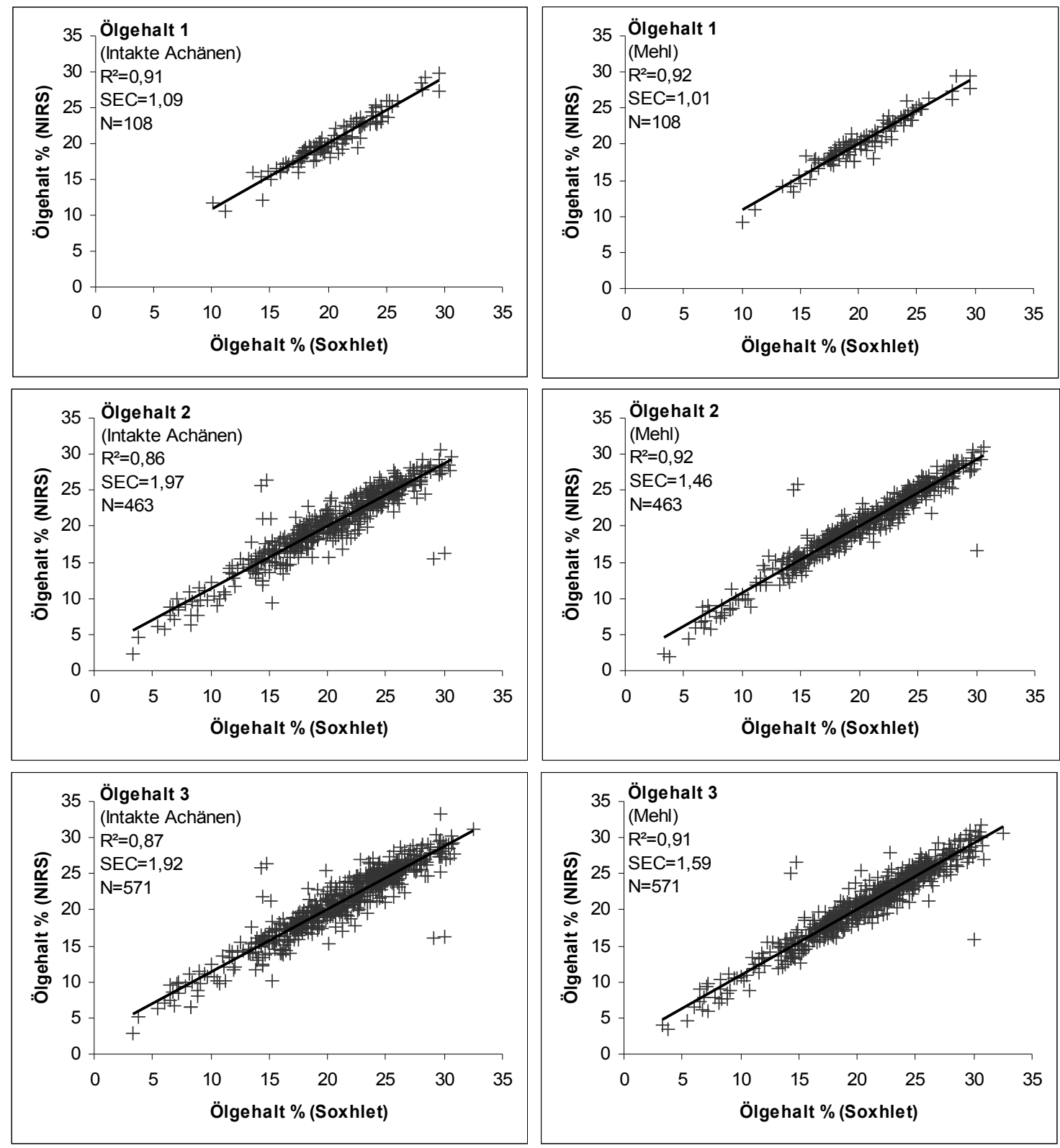

$\mathrm{R}^{2}=$ Bestimmtheitsmaß der Kalibration, SEC= Standardfehler der Kalibration, $\mathrm{N}=$ Anzahl Proben

Abb. 2: Beziehung zwischen Referenzwerten und NIRS-Werten für Ölgehalt

Die Abb. 3 zeigt die Häufigkeitsverteilung des Ölgehaltes (Soxhlet) der Proben, die in die Kalibrationen eingegangen sind. Der Probensatz wurde ständig erweitert, sodass Proben mit einem höheren und niedrigeren Ölgehalt hinzukamen. Die meisten Proben wiesen einen Ölgehalt zwischen 15 und 30\% auf. 


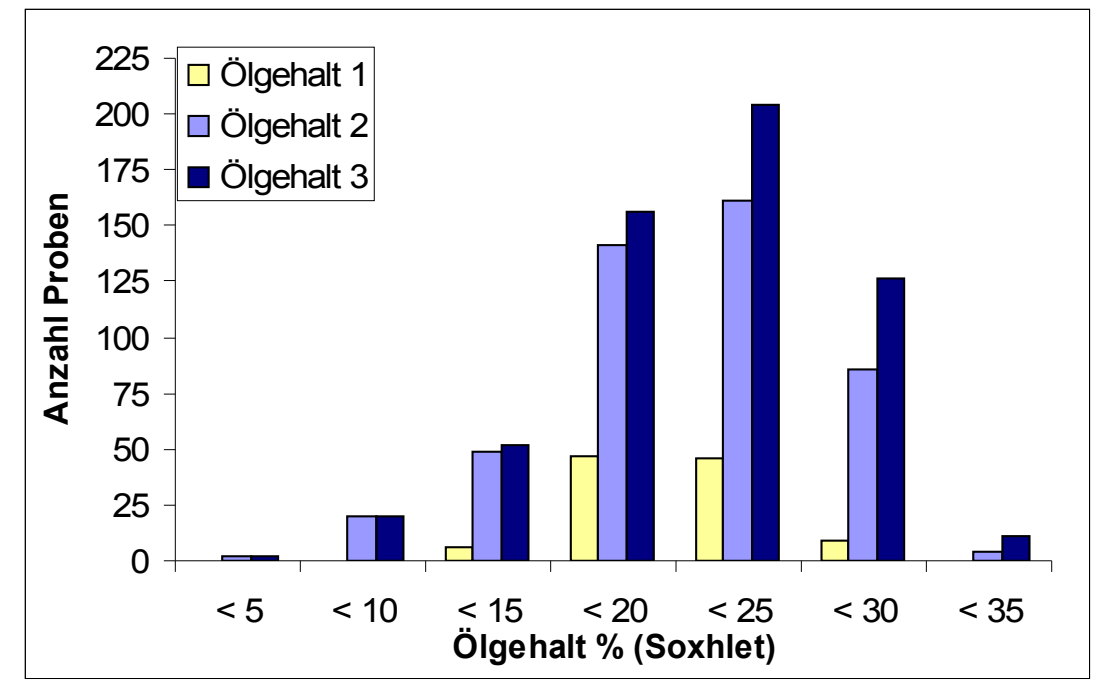

Abb. 3: Häufigkeitsverteilung der Referenzwerte (Soxhlet) für die Kalibrationen Ölgehalt 1, 2 und 3

\subsubsection{Kalibration für Linolsäure- und Ölsäuregehalt}

Die statistischen Parameter der Kalibrationsentwicklungen für intakte und gemahlene Achänen sind in der Tab. 4 dargestellt.

Die 534 Referenzwerte des Linolsäuregehaltes zeigten mit einem Minimum von $67,26 \%$ und einem Maximum von $86,02 \%$ eine relativ große Variation. Die Linolsäuregehalte wurden genauer geschätzt, wenn die NIRS-Spektren an Mehl erfasst wurden $\left(R^{2}=0,83\right.$ und $\left.1-\mathrm{V} R=0,76\right)$. Auch die Standardfehler der Kalibration und der Kreuzvalidierung waren in diesem Fall niedriger $(\mathrm{SEC}=1,38$ und SECV=1,63).

Der Ölsäuregehalt wurde mit Hilfe desselben Probensatzes geschätzt. Die Spannweite der Referenzwerte betrug hier $6,06 \%$ bis $17,81 \%$. Auch bei diesem Merkmal waren die Bestimmtheitsmaße bei der Variante Mehl höher $\left(R^{2}=0,84\right.$ und $1-V R=0,76$ ) und die Standardfehler niedriger (SEC=0,91 und SECV=1,11).

Die Genauigkeit der Kalibrationen für Linolsäure- und Ölsäuregehalt, gemessen an SD/SECV, unterschieden sich kaum. Dies galt sowohl für die Kalibrationen, die auf den Messungen der intakten Achänen beruhten, als auch für die, die aufgrund von Messungen an Mehl erstellt wurden.

Die Kalibrationen lieferten nicht so exakte Ergebnisse wie die Kalibrationen für den Ölgehalt, was die geringeren SD/SECV-Werte zeigten. 
Tab. 4: Statistik der Kalibrationen und der Kreuzvalidierungen für Linolsäureund Ölsäuregehalt (\%)

\begin{tabular}{|c|c|c|c|c|c|c|c|c|c|c|}
\hline \multirow[b]{2}{*}{ Merkmal } & \multicolumn{7}{|c|}{ Kalibration ${ }^{a}$} & \multicolumn{2}{|c|}{$\begin{array}{c}\text { Kreuz- } \\
\text { validierung }\end{array}$} & \multirow[t]{2}{*}{$\begin{array}{c}\text { SDI } \\
\text { SECV }\end{array}$} \\
\hline & $\mathbf{N}$ & Mittel & SD & Min. & Max. & SEC & $\mathbf{R}^{2}$ & SECV & $1-V R$ & \\
\hline \multicolumn{11}{|c|}{ Intakte Achänen } \\
\hline Linolsäure & 534 & 80,95 & 3,33 & 67,26 & 86,02 & 1,74 & 0,73 & 2,05 & 0,62 & 1,63 \\
\hline Ölsäure & 534 & 9,32 & 2,27 & 6,06 & 17,81 & 1,28 & 0,68 & 1,44 & 0,60 & 1,58 \\
\hline \multicolumn{11}{|l|}{ Mehl } \\
\hline Linolsäure & 534 & 80,95 & 3,33 & 67,26 & 86,02 & 1,38 & 0,83 & 1,63 & 0,76 & 2,04 \\
\hline Ölsäure & 534 & 9,32 & 2,27 & 6,06 & 17,81 & 0,91 & 0,84 & 1,11 & 0,76 & 2,04 \\
\hline
\end{tabular}

Die Referenzwerte des Linolsäure- und Ölsäuregehaltes sind in Abb. 4 graphisch den NIRS-Werten gegenübergestellt. Neben den Bestimmtheitsmaßen und den Standardfehlern ist die Verteilung der Werte über den Probensatz verdeutlicht. Beim Linolsäuregehalt wurden viele Proben mit einem Gehalt zwischen $75 \%$ und $85 \%$ für die Kalibration verwendet. In die Gleichung für Ölsäure sind hauptsächlich Proben mit einem Gehalt zwischen 6\% und 11\% eingeflossen. 

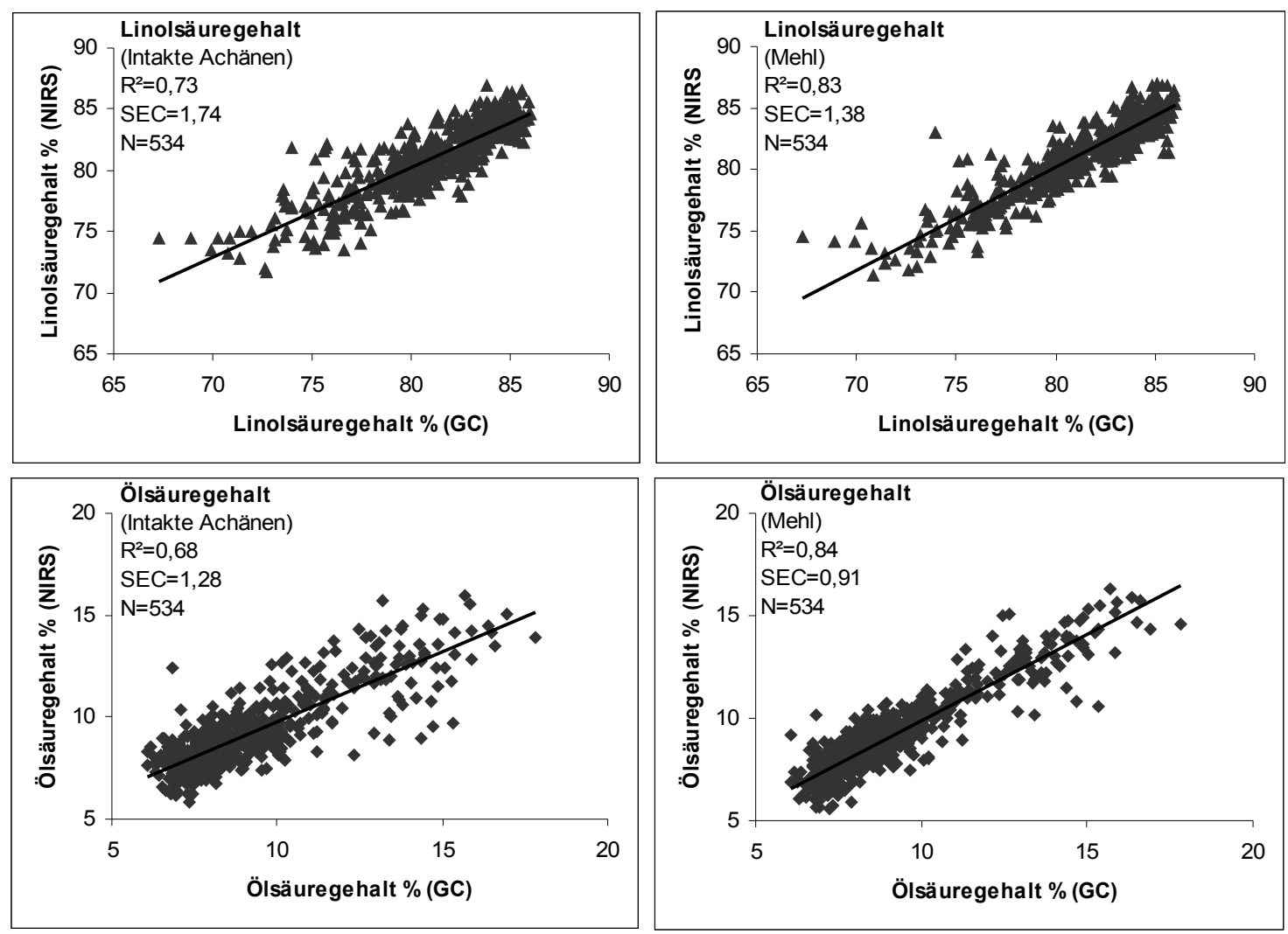

$\mathrm{R}^{2}=$ Bestimmtheitsmaß der Kalibration, $\mathrm{SEC}=$ Standardfehler der Kalibration, $\mathrm{N}=\mathrm{Anzahl}$ Proben

Abb. 4: Beziehung zwischen Referenzwerten und NIRS-Werten für Linolsäuregehalt (oben) und Ölsäuregehalt (unten)

\subsubsection{Kalibration für Schalenanteil und Anteil tauber Achänen}

Die Kalibrationen zum Schätzen des Schalenanteils beruhten auf 158 Proben, die eine Standardabweichung von 13,4 (Minimum=42,51\%, Maximum=99,10\%) zeigten. Anders als bei den Merkmalen Ölgehalt, Linolsäure- und Ölsäuregehalt lagen hier die SD/SECV-Werte bei der Gleichung für intakte Achänen höher als bei der Variante Mehl. Die Bestimmtheitsmaße der Kalibration und Kreuzvalidierung fielen bei der Messung intakter Achänen ebenfalls höher $\left(R^{2}=0,90\right.$ und $\left.1-V R=0,81\right)$ aus.

Die gleichen 158 Proben wurden auf den Anteil tauber Achänen untersucht. Hier gab es eine große Variation (Minimum $=0,00 \%$, Maximum $=95,00 \%$ ). Wie bei den Kalibrationen für den Linolsäuregehalt zeigte sich, dass die Gleichung in der Variante intakte Achänen etwas genauer war. Die SD/SECV-Werte waren bei den Kalibrationen für den Anteil tauber Achänen im Vergleich zu den Kalibrationen für den Ölgehalt relativ gering (Tab. 5). 
Tab. 5: Statistik der Kalibrationen und der Kreuzvalidierungen für Schalenanteil (\%) und Anteil tauber Achänen (\%)

\begin{tabular}{|c|c|c|c|c|c|c|c|c|c|c|}
\hline \multirow[b]{2}{*}{ Merkmal } & \multicolumn{7}{|c|}{ Kalibration ${ }^{a}$} & \multicolumn{2}{|c|}{$\begin{array}{c}\text { Kreuz- } \\
\text { validierung }\end{array}$} & \multirow[t]{2}{*}{$\begin{array}{l}\text { SDI } \\
\text { SECV }\end{array}$} \\
\hline & $\mathbf{N}$ & Mittel & SD & Min. & Max. & SEC & $\mathbf{R}^{2}$ & SECV & 1-VR & \\
\hline \multicolumn{11}{|l|}{ Intakte Achänen } \\
\hline Schalenanteil & 158 & 63,33 & 13,40 & 42,51 & 99,10 & 4,27 & 0,90 & 5,81 & 0,81 & 2,31 \\
\hline Taube Achänen & 158 & 17,50 & 23,34 & 0,00 & 95,00 & 9,33 & 0,84 & 12,09 & 0,73 & 1,93 \\
\hline \multicolumn{11}{|l|}{ Mehl } \\
\hline Schalenanteil & 158 & 63,33 & 13,40 & 42,51 & 99,10 & 6,03 & 0,80 & 6,66 & 0,75 & 2,01 \\
\hline Taube Achänen & 158 & 17,50 & 23,34 & 0,00 & 95,00 & 11,33 & 0,76 & 12,78 & 0,70 & 1,83 \\
\hline
\end{tabular}

In Abb. 5 sind die Referenzwerte den NIRS-Werten für den Schalenanteil und den Anteil tauber Achänen gegenübergestellt. Für beide Merkmale wurden mehr Proben aus den niedrigen Bereichen zur Kalibrationsentwicklung hinzugezogen (Schalenanteil: $50 \%$ bis $60 \%$, Anteil tauber Achänen: $0 \%$ bis $20 \%)$.
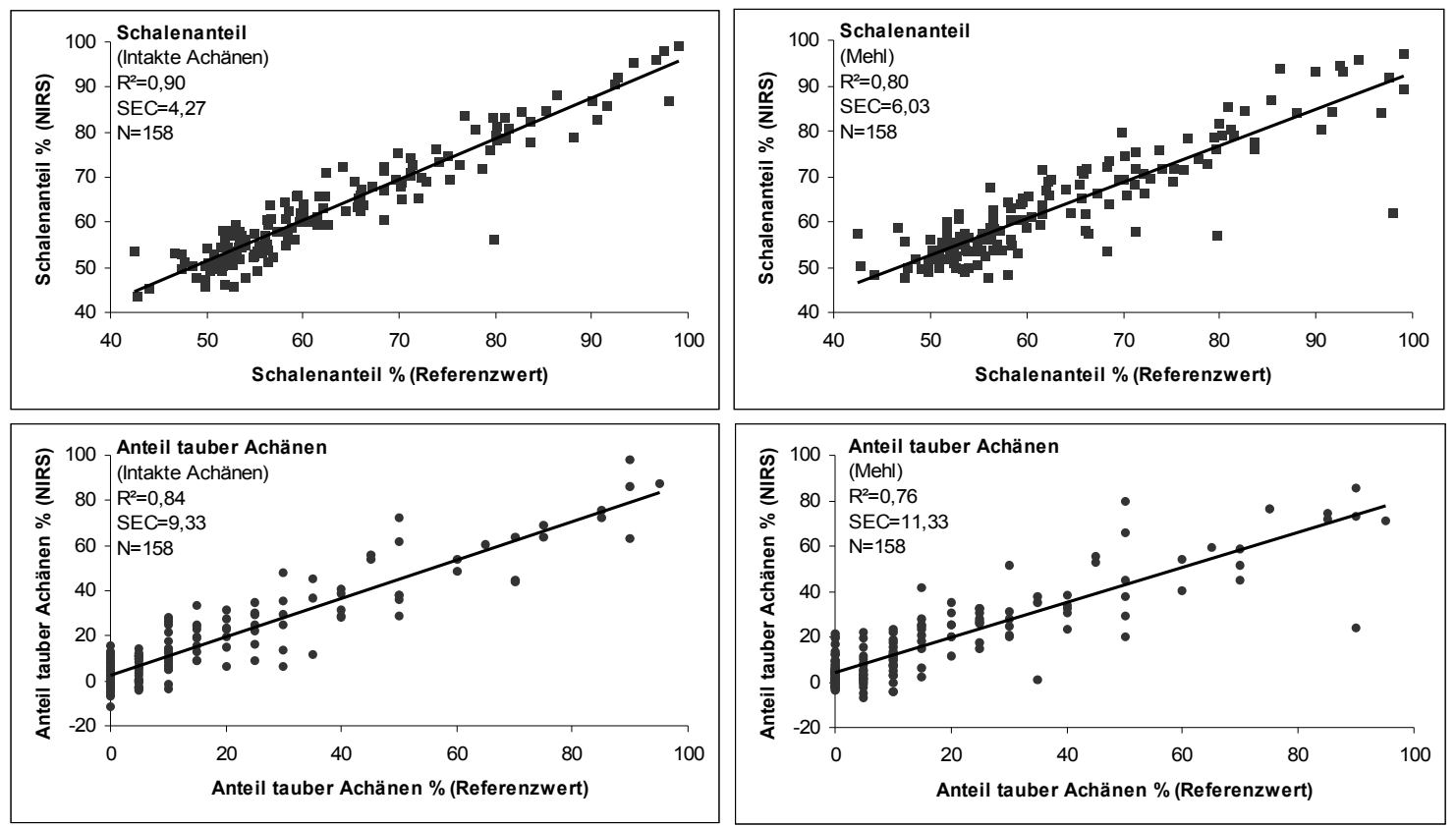

$\mathrm{R}^{2}=$ Bestimmtheitsmaß der Kalibration, SEC= Standardfehler der Kalibration, N=Anzahl Proben

Abb. 5: Beziehung zwischen Referenzwerten und NIRS-Werten für Schalenanteil (oben) und Anteil tauber Achänen (unten) 


\subsubsection{Merkmalskorrelationen}

Eine hoch signifikante, negative Beziehung wurde zwischen dem Ölgehalt und dem Schalenanteil festgestellt $\left(-0,832^{* *}\right)$. Auch zwischen dem Anteil tauber Achänen und dem Ölgehalt zeigte sich eine hoch signifikante, negative Korrelation $\left(-0,702^{* *}\right)$. Ein positiver, hoch signifikanter Wert wurde für die Korrelation zwischen Schalenanteil und Anteil tauber Achänen errechnet $\left(0,892^{* *}\right)$. Die beiden Fettsäuren Linolsäure und Ölsäure korrelierten in ihrem Gehalt hoch signifikant negativ $\left(-0,826^{* *}\right)$. Der Ölgehalt zeigte eine hoch signifikante, positive Beziehung zum Linolsäuregehalt und eine hoch signifikante, negative zum Ölsäuregehalt (Tab. 6).

Tab. 6: Spearman's Rangkorrelationen zwischen den Merkmalen Ölgehalt, Linolsäure- und Ölsäuregehalt, Schalenanteil und Anteil tauber Achänen $(\mathrm{N}=146)$

\begin{tabular}{lcccc}
\hline Merkmal & Ölgehalt & Linolsäure & Ölsäure & Schalenanteil \\
\hline Linolsäure & $0,503^{* *}$ & & & \\
Ölsäure & $-0,371^{* *}$ & $-0,826^{* *}$ & & \\
Schalenanteil & $-0,832^{* *}$ & $-0,455^{* *}$ & $0,348^{* *}$ & \\
Taube Achänen & $-0,702^{* *}$ & $-0,391^{* *}$ & $0,308^{* *}$ & $0,892^{* *}$ \\
\hline
\end{tabular}

** signifikant bei $p=0,01$ 


\subsection{Diskussion}

Verschiedene Studien haben gezeigt, dass NIRS eine einfache, schnelle und kostengünstige Methode darstellt, um Inhaltsstoffe und andere Qualitätsparameter mit nur einer Messung an ganzen Körnern oder Mehl zu schätzen. Anhand von intakten Saflorachänen und Mehlproben wurden verschiedene Kalibrierungen für die Merkmale Ölgehalt, Linolsäure- und Ölsäuregehalt, Schalenanteil und Anteil tauber Achänen entwickelt.

\subsubsection{NIRS für Ölgehalt}

Die statistischen Parameter der Kalibrationsgleichungen für den Ölgehalt zeigten von allen untersuchten Merkmalen die besten Ergebnisse. Die Kalibrationen für Mehl wiesen geringere Standardfehler und höhere Bestimmtheitsmaße als diejenigen für intakte Samen auf. Generell wird NIRS zur Untersuchung von ganzen, auch einzelnen, Samen benutzt (Velasco et al. 1999 a, b). Pazdernik et al. (1997) schätzten Aminosäure- und Fettsäurezusammensetzungen der Sojabohne mittels NIRS. Sie berichteten, dass die NIRS-Methode genauere Ergebnisse lieferte, wenn die Samen vor der Spektrenaufnahme gemahlen wurden.

Bei NIRS-Untersuchungen von Sonnenblumenachänen wurden für den Ölgehalt sowie für verschiedene Fettsäuregehalte ebenfalls exaktere Ergebnisse mit gemahlenen Achänen erzielt (Pérez-Vich et al. 1998). Zu dem gleichen Schluss kamen Moschner et al. (2004). Die unterschiedliche Form und Größe könnte bei der Spektrenaufnahme intakter Achänen ein Problem darstellen (Baye und Becker 2004). Besonders könnte sich, wie bei der Sonnenblume, die Dicke der Schalen störend auf die Spektrenaufnahme auswirken. Saflorachänen besitzen einen ähnlichen Schalenanteil wie Sonnenblumenachänen und Inhaltsstoffe wie Öl und Fettsäuren befinden sich im Embryo (Scheibe 1938).

Die Kalibrationen Ölgehalt 2 und 3 zeigten in beiden Varianten (intakte und gemahlene Achänen) höhere Standardfehler und geringere Bestimmtheitsmaße bei einer größeren Variation der Referenzwerte. Dennoch wurden diese erweiterten Kalibrationen in den betreffenden Jahren angewendet (siehe Kapitel 3), da die Kalibrationen Ölgehalt 1 hauptsächlich auf Proben von einem Ort und Jahr beruhten und daher nur für das Jahr 2004 und den Ort Göttingen 
präzise Ergebnisse lieferten. Die Erweiterung eines Kalibrationssets durch Proben verschiedener Umwelten sollte nach Velasco et al. (1998) zu einer robusteren Kalibration führen. Eine Tendenz zu einer robusteren Kalibrierung war bei der Variante intakte Achänen von der Kalibration Ölgehalt 2 zu der erweiterten Ölgehalt 3 zu erkennen. In der Kreuzvalidierung verringerte sich der Standardfehler etwas und das Bestimmtheitsmaß nahm zu (2005: SECV=2,18, 1-VR=0,83; 2006: SECV=2,13, 1-VR=0,84). In der Variante Mehl war diese Tendenz nicht zu erkennen.

Generell kann NIRS als eine schnelle, kostengünstige Methode angewendet werden, um den Ölgehalt in einer großen Anzahl von Saflorproben zu untersuchen, zum Beispiel bei der Selektion vieler Genotypen, die eine große Variation aufweisen. Werden genaue Ergebnisse benötigt, sollte anschließend die kleinere Gruppe der besten, mittels NIRS ausgewählten, Genotypen im Labor untersucht werden. NIRS kann folglich dazu dienen, eine anfangs große Anzahl Proben für die zeitaufwändigen Laboranalysen zu reduzieren. Besonders interessant ist die Kalibration für intakte Achänen, da durch diese Methode der Ölgehalt auch bei kleinen Saatgutmengen bestimmt werden kann, ohne die Achänen zu vermahlen.

Die Kalibrationen Ölgehalt 3 sollten für die Messung von Probensätzen anderer Erntejahre und Orte durch weitere Proben dieser Orte und Jahre ergänzt werden, um die Genauigkeit zu verbessern. Möglicherweise könnten durch die Verwendung größerer Küvetten, die eine größere Probenmenge enthalten und eine größere Oberfläche besitzen, die Standardfehler verringert werden.

\subsubsection{NIRS für Linolsäure- und Ölsäuregehalt}

Die Bestimmtheitsmaße verdeutlichten besonders in der Variante für intakte Achänen, dass die mittels NIRS geschätzten Werte mit den Referenzwerten nur bedingt übereinstimmten. Wie beim Ölgehalt wurde eine deutliche Verbesserung der Schätzgenauigkeit erreicht, wenn Spektren von Mehl erfasst wurden. Die Bestimmtheitsmaße waren größer und die Standardfehler geringer. Dieses Ergebnis wurde auch in Studien an Sojabohne (Pazdernik et al. 1997) und Sonnenblume (Pérez-Vich et al. 1998, Moschner et al. 2004) gefunden. Genotypen mit niedrigen Linolsäuregehalten (zwischen $67 \%$ und $77 \%$ ) und hohen Ölsäuregehalten (zwischen $10 \%$ und 18\%) wiesen größere Abweichungen zwischen den Referenzwerten und den Schätzwerten auf (siehe 
Abb. 4). Insgesamt waren nur geringfügige Unterschiede in der Schätzgenauigkeit für Linolsäure- und Ölsäuregehalt erkennbar.

Die NIRS-Methode kann aufgrund dieser Ergebnisse in der Variante "Messung intakter Achänen" als eine nicht destruktive, grobe Schätzmethode für Linolsäure- und Ölsäuregehalt angewendet werden. Bessere Ergebnisse wurden durch die Messung von Mehl erzielt, die aber wahrscheinlich nicht an die Präzision der GC-Analyse reichten. Hilfreich kann die NIRS-Messung sein, um eine Vorauswahl der Proben zu treffen, die im Labor untersucht werden sollen. Durch Hinzufügen weiterer Proben aus anderen Jahren und Orten könnten die Kalibrationen, deren Standardabweichungen bislang noch relativ gering sind, möglicherweise verbessert werden.

\subsubsection{NIRS für Schalenanteil und Anteil tauber Achänen}

Die Kalibrationen für Schalenanteil und den Anteil tauber Achänen wurden anhand eines Probensatzes mit einer hohen Standardabweichung entwickelt (Schalenanteil: $S D=13,40$, Anteil tauber Achänen: $S D=23,34$ ). Die besonders hohen Werte im Schalenanteil und Anteil tauber Achänen zeigten, dass in diesem Probensatz Genotypen vorhanden waren, deren Achänen nicht vollständig ausgebildet waren. Um eine breite Variation für die Kalibrierung zu erhalten und später auch solche Proben messen zu können, wurden diese Genotypen ebenfalls mit einbezogen.

In der Variante Mehl ergaben sich hier geringere Bestimmtheitsmaße, während die Standardfehler höher waren. Die NIRS-Werte stimmten besser überein, wenn die Gleichung auf der Grundlage intakter Achänen entwickelt wurde. Dies stand im Gegensatz zu den Daten der Kalibrationen für den Ölgehalt, Linolsäure- und Ölsäuregehalt, bei denen genauere Ergebnisse mit der Variante Mehl erzielt wurden. Es wäre auch hier zu erwarten gewesen, dass in der Variante Mehl genauere Ergebnisse erzielt werden, da die Oberfläche wesentlich homogener ist als bei der Variante intakte Achänen.

Die Standardfehler waren besonders in den Kalibrationen zum Schätzen des Anteils tauber Achänen sehr hoch. Die NIRS-Schätzungen reichten zum Teil in den negativen Bereich (siehe Abb. 5). 
Die NIRS-Methode kann bei Saflor für diese Merkmale keine exakte Schätzung liefern. Bei einer großen Anzahl von Proben mit breiter Variation sind diese Kalibrationen für Informationen über den Schalenanteil und den Anteil tauber Achänen hilfreich, da die Bestimmung durch Einweichen und Wiegen der Achänen sich als sehr zeitaufwändig darstellte.

Die Kalibrationen beruhten allerdings auf nur 158 Proben aus Göttingen. Das Hinzufügen von Proben weiterer Orte und Jahre würde die Aussagekraft der Kalibrationen vermutlich verbessern.

Aufgrund des hohen Standardfehlers in den Kalibrationen zum Schätzen des Anteils tauber Achänen wurde dieses Merkmal in der weiteren Arbeit nicht genauer verfolgt.

\subsubsection{Merkmalskorrelationen}

Die enge negative Korrelation zwischen dem Ölgehalt und dem Schalenanteil sowie die zwischen dem Ölgehalt und dem Anteil tauber Achänen waren zu erwarten. Knowles (1969) berichtete, dass der Ölgehalt bei reduziertem Schalenanteil zunahm. Klisiewicz und Urie (1982) erwähnten ebenfalls einen geringen Ölgehalt infolge eines hohen Schalenanteils. Auch der Zusammenhang zwischen dem Schalenanteil und dem Anteil der tauben Achänen wurde bereits im Vorfeld vermutet.

Die sehr starke negative Korrelation zwischen dem Linolsäure- und Ölsäuregehalt wurde in anderen Arbeiten mit Saflor ebenfalls gefunden (Johnson et al. 1999, Fernández-Martínez et al. 1993).

Im Gegensatz zu anderen Beobachtungen stand die positive Korrelation zwischen dem Ölgehalt und dem Linolsäuregehalt, sowie die negative Beziehung zwischen dem Ölgehalt und dem Ölsäuregehalt. Johnson et al. (1999) beschrieb bei Saflor eine negative Beziehung zwischen dem Linolsäuregehalt und dem Ölgehalt $\left(-0,20^{* *}\right)$ und eine positive zwischen dem Ölgehalt und dem Ölsäuregehalt $\left(0,20^{* *}\right)$. Der Ölsäuregehalt zeigte in dieser Studie, in der 1000 Proben analysiert wurden, eine große Variation $(7 \%$ bis $84 \%$ ). In der vorliegenden Arbeit wurden nur 158 Proben mit einer Variation von $7 \%$ bis $16 \%$ Ölsäuregehalt untersucht. Auch im Raps wurde eine positive Korrelation zwischen Ölgehalt und Ölsäuregehalt (bei normalem Raps: 0,48** und bei Hochölsäuremutanten: $0,42^{*}$ ) gefunden. In diesen Untersuchungen 
betrug die Spannweite im Ölsäuregehalt 56\% bis 75\% (Möllers und Schierholt 2002).

\subsubsection{Schlussfolgerung}

Die Ergebnisse zeigen, dass NIRS für die Bestimmung des wichtigsten Qualitätsmerkmals von Saflor, des Ölgehaltes, in der Züchtung eine geeignete Methode darstellt. Bei der Untersuchung vieler Proben kann mit Hilfe der NIRSMethode eine Vorauswahl sogar an intakten Achänen getroffen werden. Es ist nicht notwendig, die oft geringen Saatgutmengen zu vermahlen.

Durch den Vergleich der SD/SECV-Werte der Kalibrationen wird deutlich, dass die Methode bei der Bestimmung des Linolsäure- und Ölsäuregehaltes sowie des Schalenanteils und des Anteils tauber Achänen weniger präzise Ergebnisse liefert als bei dem Ölgehalt.

Der Linolsäure- und Ölsäuregehalt stehen bei dem in Deutschland kaum züchterisch bearbeiteten Saflor in der Gewichtung der Qualitätsmerkmale vorläufig hinter dem ÖIgehalt. Die Variation im Fettsäuremuster war bei den untersuchten Proben der vorliegenden Arbeit nicht besonders groß. Es wurden nur Linolsäuretypen einbezogen, die zur Speiseölgewinnung dienen. Ölsäuretypen besaßen für das Ziel dieser Arbeit keine Relevanz. Interessant wären möglicherweise weiterführende Untersuchungen, ob mittels NIRS bei Saflor Linolsäuretypen und Ölsäuretypen unterschieden werden können.

Der geringere Schalenanteil stellt laut Scheibe (1939) ein wichtiges Zuchtziel dar, um den Ölgehalt zu erhöhen. Der Ölgehalt war stark negativ mit dem Schalenanteil korreliert. Da es durch NIRS möglich ist, den Ölgehalt schnell und kostengünstig zu bestimmen, verliert die Bestimmung des Schalenanteils an Wichtigkeit.

Für den folgenden Teil der Arbeit, der sich mit der Untersuchung der verschiedenen Züchtungsmethoden befasst, stellte NIRS eine einfache und praktikable Methode zur Erfassung des Ölgehaltes dar. 


\section{Verfahren der Auslese}

\subsection{Einleitung}

Saflor kann einen Ölgehalt von bis zu 45\% erzielen (Dajue und Mündel 1996). Der Anbau wäre in Deutschland möglich, aber unter mitteleuropäischen Verhältnissen erweisen sich die Sorten mit hohem Ölgehalt als stark krankheitsanfällig, was zu einem völligen Ertragsausfall führen kann (Biertümpfel et al. 2006). In Deutschland wurde bislang fast keine Züchtung für Saflor durchgeführt, sodass das Ziel der vorliegenden Arbeit war, drei Züchtungsmethoden (Stammbaummethode, natürliche Auslese und Einkornramschmethode) bei Saflor in Deutschland zu untersuchen und zu beurteilen. Besonders wurde auf die Zuchtziele Ölgehalt und geringe Krankheitsanfälligkeit geachtet.

Meist werden Variationen der Stammbaummethode für die Selektion auf hoch heritable Merkmale wie Frühreife und Krankheitsresistenz bei Saflor angewendet (Knowles 1989). In segregierenden Populationen werden F2Pflanzen für die Beurteilung als F3 ausgewählt und in Reihen angebaut. In der F3 werden zunächst die besten Reihen selektiert und innerhalb der Reihen die besten Einzelpflanzen. In der F4 werden die Nachkommen der selektierten F3Pflanzen nach Verwandtschaft geordnet angebaut. Die Selektion findet dann in drei Schritten statt. Erst wird zwischen den Familien, dann innerhalb der Familien und zum Schluss werden innerhalb der Reihen die besten Einzelpflanzen selektiert. In späteren Generationen werden keine Einzelpflanzen mehr selektiert, sondern die ganzen Reihen geerntet und getestet. Das Prinzip dieser Methode beruht darauf, das Potential der Einzelpflanze anhand ihrer Verwandtschaft zu beurteilen (Becker 1993). Knowles (1989) beschrieb für Saflor die Methode der Stammbaummethode mit frühen Tests. Schon in frühen Generationen werden Ertragstests durchgeführt, indem einzelne F3-Pflanzen in der Reihe für die Beurteilung als F4 geselbstet werden und das restliche, offen abgeblühte Saatgut der Reihe für Ertragstests genutzt wird.

Die natürliche Auslese wird bei Saflor in den USA seit den 1960er Jahren für die Selektion auf Krankheitsresistenz verwendet. Mehrere Sorten wurden auf der Grundlage dieser Methode entwickelt (Mündel et al. 1985a, Bergmann et al. 
1985, 1987, 1989). Das Saatgut der gesamten F2 wird in einer Parzelle ausgesät. Jeweils eine Stichprobe des Erntegutes kommt in den folgenden Generationen erneut zur Aussaat. Die Ramschgenerationen unterliegen nur der natürlichen Auslese. In der Ramschmethode werden nach der natürlichen Selektion, wenn ein ausreichend hoher Homozygotiegrad erreicht ist, die Nachkommen einzelner Pflanzen reihenweise angebaut und selektiert (Becker 1993).

Nach Fernández-Martínez et al. (1986) eignet sich die Einkornramschmethode als eine zeitsparende und effektive Möglichkeit für die Züchtung von Saflor. In dieser Methode wird anfangs nicht selektiert. In jeder Generation wird eine Achäne jeder Pflanze zufällig geerntet und wieder ausgesät. Da Umweltbedingungen unbedeutend sind, kann der Einkornramsch zeitsparend im Gewächshaus durchgeführt werden. Haben die Pflanzen einen ausreichenden Homozygotiegrad erreicht, werden sie als Reihen auf dem Feld angebaut und selektiert (Becker 1993).

Ein Schema der drei Züchtungsmethoden, wie sie in der vorliegenden Arbeit durchgeführt wurden, ist in der Abb. 6 dargestellt. 


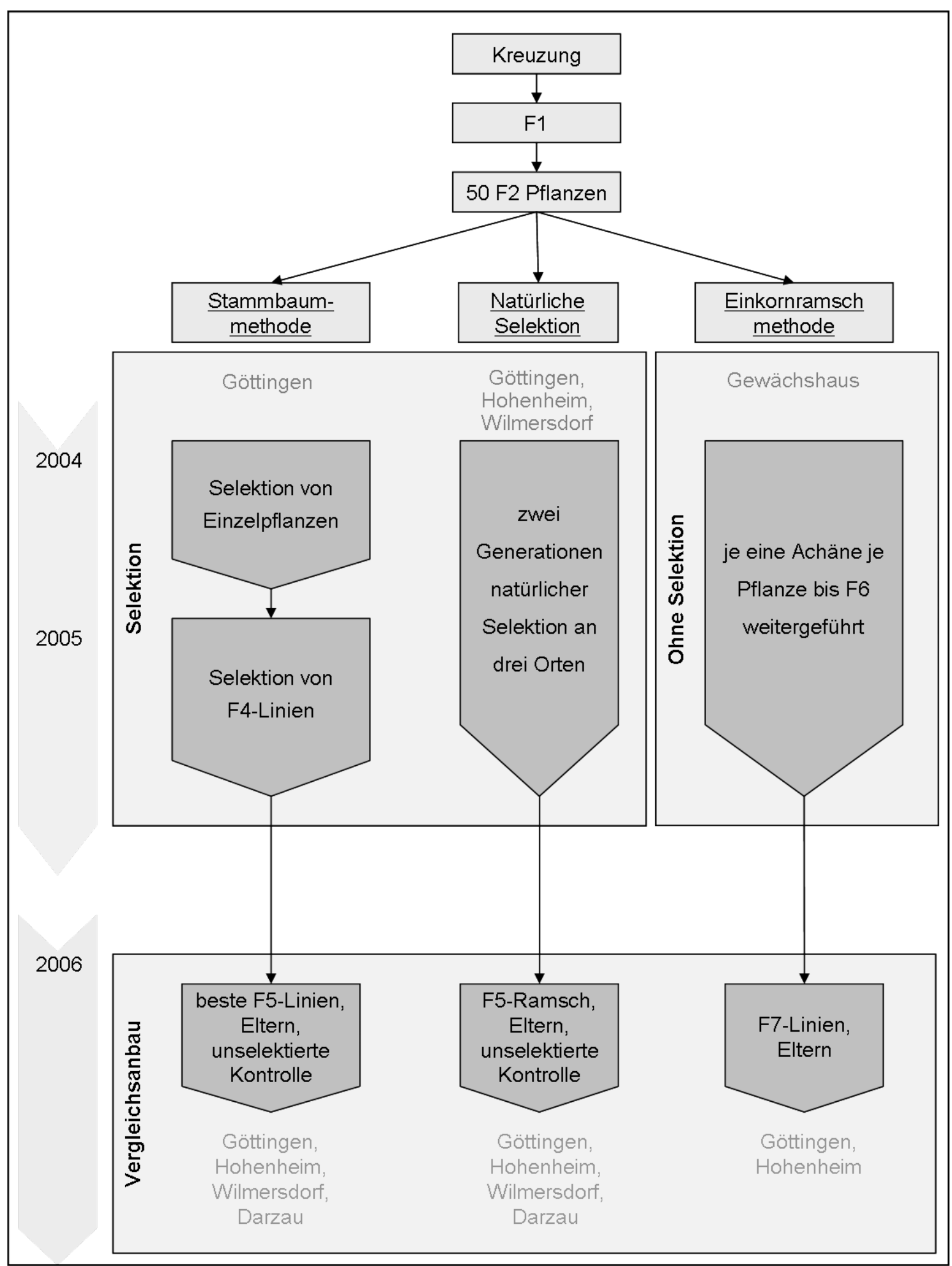

Abb. 6: Schema des Versuchsaufbaus 
Hohe Luftfeuchtigkeit und Niederschläge in und nach der Zeit der Blüte des Saflors begünstigen Krankheiten. Die wichtigsten Erreger sind: Alternaria carthami Chowdhury (Alternaria-Blattflecken), Ramularia carthami Zaprometov (Ramularia-Blattflecken), Fusarium oxysporum Schlecht. f. carthami Klis. \& Hous. (Fusarium), Puccinia carthami Cda. (Saflorrost), Pseudomonas syringae Van Hall (bakterielle Fäule) und Botrytis cinerea Pers. ex. Fr. (Köpfchenfäule) (Knowles 1989).

Bei den Untersuchungen zu dieser Arbeit traten hauptsächlich Köpfchenfäule, Alternaria-Blattflecken, Saflorrost und 2006 zusätzlich Fusarium auf.

Die Köpfchenfäule gilt als die wichtigste und ertragsrelevanteste Krankheit beim Saflor. Mündel et al. (2004) nannten Sclerotinia sclerotiorum (Lib.) de Bary als Erreger der Köpfchenfäule in Kanada. Spaar et al. (1990) beschrieben den Erreger Botrytis cinerea für die Köpfchenfäule. Auch bei Studien mit Saflor in der Schweiz wurde Botrytis cinerea nachgewiesen (Frick und Hebeisen 2005). Schon 1939 beschrieb Scheibe die Symptome. Die infizierten Blütenköpfe verbleichen und der Blütenboden vermorscht, sodass sich die vergilbten Blütenköpfe leicht abheben lassen. Die Köpfchenfäule tritt häufig nach der Blüte auf, die Sporen, die sich durch Wind schnell verbreiten, sind jedoch oftmals auch schon vorher nachweisbar. Resistente Sorten sind nicht bekannt (Kaffka und Kearney 1998).

Alternaria-Blattflecken treten, wie die Köpfchenfäule, in Gegenden mit hohem Niederschlag auf. Alternaria carthami ist ein bedeutender, samenbürtiger Erreger bei Saflor. Die Symptome der Krankheit treten vor der Blüte auf den Blättern als braune bis dunkelbraune Flecken auf. Der Erreger befällt anschließend auch die Blütenköpfe. Die infizierten Samen sind verschrumpelt, taub und braun verfärbt. Ein reduzierter Ölgehalt und geringer Ertrag können die Folge sein (Mündel et al. 1997, Mündel et al. 2004). In Indien wurden resistente Genotypen gefunden (Deokar et al. 1999).

Saflorrost (Puccinia carthami) tritt weltweit auf. Es handelt sich um einen boden- und samenbürtigen, durch Wind verbreiteten Erreger. Starke Kontamination mit Rost kann zu einer eingeschränkten Keimfähigkeit führen (Mündel et al. 2004). 
Auch Fusarium kann zu erheblichen Ertragsverlusten führen (Sastry und Chattopadhyay 2003). Die Symptome reichen von Welke und Vergilben bis hin zum Absterben der Pflanze. Der Erreger überdauert im Boden und am Samen (Kaffka und Kearney 1998, Mündel und Huang 2003). Es wurden resistente Genotypen gefunden (Klisiewicz und Urie 1982). 


\subsection{Material und Methoden}

\subsubsection{Pflanzenmaterial}

In dieser Arbeit wurden drei Kreuzungen untersucht, an denen vier Eltern beteiligt waren. Es wurden an das europäische Klima angepasste Genotypen mit kanadischen Genotypen mit hohem Ölgehalt gekreuzt:

- AC Sunset x CR1 (Kreuzung 1)

- $\quad$ Saffire x CR1 (Kreuzung 2)

- Sabina x AC Sunset (Kreuzung 3)

Die Sorte „Sabina“ wurde von der Norddeutschen Pflanzenzucht Hans-GeorgLembke KG, Hohenlieth, 1997 zugelassen. Der Ursprungszüchter ist das Research Institute for Fodder Crops Ltd. in Troubsko (Tschechien) (http://www.vupt.cz). Die Sorte wurde aus stachellosen Genotypen österreichischer und tschechischer Genbanken selektiert und ist an die klimatischen Bedingungen in Deutschland gut angepasst. Sie erzielt hohe Kornerträge, ihr Ölgehalt ist jedoch mit 25\% gering (Reinbrecht et al. 2004).

In einer Parzelle der Sorte Sabina wurde von Dr. C. Reinbrecht, Universität Hohenheim, ein weiterer Genotyp gefunden, im folgenden „CR1“ genannt. Phänotypisch ähnelt er der Sorte Sabina, ist jedoch bestachelt. Dieser Genotyp ist ebenfalls als Kreuzungselter verwendet worden.

Zwei kanadische Sorten, beide gezüchtet an der Agriculture Canada Research Station, Lethbridge, Alberta, wurden als weitere Eltern gewählt. Die Sorte „Saffire“ ist für die Anbauregion Südkanada entwickelt worden. Sie kombiniert eine frühe Abreife mit einem hohen Samenertrag, einem hohen Ölgehalt und einer guten Resistenz gegen Sclerotinia sclerotiorum. Mündel et al. 2004 berichteten jedoch von einer Anfälligkeit gegen Alternaria carthami, dem Erreger der Alternaria-Blattflecken. Bei Studien in der Schweiz zeigte sich die Sorte Saffire als anfällig für den Erreger Botrytis cinerea (Köpfchenfäule) (Frick und Hebeisen 2005). Es wird ein durchschnittlicher Ölgehalt von 31,8\% angegeben (Mündel et al. 1985 a, b; Mündel und Braun 1999). 
Die zweite kanadische Sorte „AC Sunset“ erzielt einen noch höheren Ölgehalt (34\%) und eine gute Resistenz gegen Sclerotinia sclerotiorum. Sie wird jedoch als anfällig für Alternaria-Blattflecken und Puccinia carthami, den Erreger für Rostbefall, beschrieben (Mündel 1996). Bei Frick und Hebeisen (2005) war AC Sunset sehr anfällig für Botrytis cinerea (Köpfchenfäule).

Die Kreuzungen wurden im Sommer 2002 an der Universität Hohenheim von Dr. C. Reinbrecht im Feld nach der Methode von Dajue und Mündel (1996) durchgeführt. Bei dieser Methode werden gut entwickelte Köpfchen kurz vor der Blüte mit einem Plastikbeutel bedeckt. Die Temperatur und Feuchtigkeit, die in dem Beutel entsteht, verhindert das Aufplatzen der Antheren. Sind die Köpfchen zur Hälfte aufgeblüht, wird morgens der Beutel entfernt, die Blüten werden mit dem Pollen des Kreuzungselters bestäubt und der Beutel wird wieder geschlossen. Dieser Vorgang wird drei Tage hintereinander wiederholt, um möglichst hohen Samenansatz zu erhalten. Zum Ende der Blüte ersetzt eine Papiertüte den Plastikbeutel, damit sich weniger Feuchtigkeit ansammelt und die Anfälligkeit für Krankheiten reduziert wird.

Die F1-Pflanzen wurden im Frühjahr 2003 auf dem Feld angebaut und geselbstet. Im Herbst 2003 wurden 50 F2-Pflanzen jeder Kreuzungskombination im Gewächshaus ausgesät und geerntet. Anhand von morphologischen Merkmalen wie Blütenfarbe, Pflanzenlänge und Blühzeitpunkt wurde überprüft, dass es sich um spaltende F2-Populationen handelte.

\subsubsection{Standorte}

Die Freilandversuche wurden 2004, 2005 und 2006 auf ökologisch bewirtschafteten Flächen in Göttingen (südliches Niedersachsen), Hohenheim bei Stuttgart (Baden-Württemberg) und Wilmersdorf bei Angermünde (Brandenburg) angebaut. Für 2006 wurde als zusätzlicher Standort Darzau (nordöstliches Niedersachsen) gewählt.

Das Klostergut Reinshof der Universität Göttingen befindet sich $150 \mathrm{~m}$ ü. NN. Der Boden besteht aus etwa $80 \%$ Auenböden (Lehme bis tonige Lehme) aus Schwemmlöß und 20\% Grieserden aus Löß mit einer durchschnittlichen Ackerzahl von 83 BP. Der langjährige Durchschnitt der Niederschläge beträgt $645 \mathrm{~mm}$, der der Jahrestemperatur $8,7^{\circ} \mathrm{C}$. 
Das Versuchsgut Kleinhohenheim der Universität Hohenheim liegt $435 \mathrm{~m}$ ü. NN. Der Lehm-, teilweise Lößlehmboden mit einer Bodenzahl von 47-65 BP ist relativ inhomogen. Bei einer mittleren Jahrestemperatur von $8,5{ }^{\circ} \mathrm{C}$ fallen durchschnittlich $700 \mathrm{~mm}$ Niederschlag im Jahr.

In Wilmersdorf (50 m ü. NN) wurden die Versuche vom VERN e.V. (Verein zur Erhaltung und Rekultivierung von Nutzpflanzen in Brandenburg) betreut. Es handelt sich dort um sandig-lehmige Braunerden mit 25-55 BP. Der langjährige Jahresniederschlag beträgt $486 \mathrm{~mm}$, die durchschnittliche Jahrestemperatur liegt bei $8,4^{\circ} \mathrm{C}$ (Daten vom nahegelegenen Leibniz-Zentrum für Agrarlandforschung, Dedelow).

Das Gut Darzau (Getreidezüchtungsforschung Darzau) liegt auf einer Höhe von $60 \mathrm{~m}$ ü. NN. Die Bodenzahlen der Parabraunerde liegen bei 35 BP. Es fallen im langjährigen Mittel $600 \mathrm{~mm}$ Niederschlag bei einer Jahresdurchschnittstemperatur von $8,9^{\circ} \mathrm{C}$.

Die durchschnittliche Monatstemperatur und die Summe der monatlichen Niederschläge der einzelnen Orte in den Vegetationsperioden 2004 bis 2006 sind den Tab. 7 und 8 zu entnehmen.

Tab. 7: Durchschnittliche Monatstemperatur $\left({ }^{\circ} \mathrm{C}\right)$ über die Vegetationsperioden 2004 bis 2006 an den vier Standorten

\begin{tabular}{|c|c|c|c|c|c|c|c|c|c|c|}
\hline \multirow[b]{2}{*}{ Monat } & \multicolumn{3}{|c|}{2004} & \multicolumn{3}{|c|}{2005} & \multicolumn{4}{|c|}{2006} \\
\hline & Gö & Ho & $\mathbf{W i}$ & Gö & Ho & $\mathbf{W i}$ & Gö & Ho & Wi & $\mathrm{Da}$ \\
\hline März & 4,5 & 4,7 & 4,2 & 4,6 & 4,8 & 1,6 & 2,2 & 3,0 & 0,0 & 0,2 \\
\hline April & 9,1 & 10,2 & 8,5 & 9,3 & 10,2 & 8,1 & 7,8 & 9,0 & 8,1 & 7,3 \\
\hline Mai & 11,0 & 12,0 & 11,6 & 12,2 & 13,8 & 12,4 & 12,5 & 13,9 & 12,6 & 12,9 \\
\hline Juni & 14,9 & 16,2 & 14,3 & 15,1 & 18,2 & 15,2 & 16,3 & 17,6 & 17,0 & 16,4 \\
\hline Juli & 16,1 & 18,2 & 16,3 & 17,9 & 18,4 & 18,5 & 21,7 & 22,4 & 21,9 & 21,8 \\
\hline Aug. & 18,5 & 19,0 & 18,4 & 18,5 & 16,2 & 16,0 & 18,0 & 15,2 & 16,9 & 17,0 \\
\hline Sept. & 13,9 & 15,1 & 13,6 & 15,1 & 15,7 & 15,0 & 16,6 & 18,0 & 16,6 & 17,6 \\
\hline Mittelwert & 12,6 & 13,6 & 12,4 & 13,2 & 13,9 & 12,4 & 13,6 & 14,2 & 13,3 & 13,3 \\
\hline
\end{tabular}

Gö: Göttingen, Ho: Hohenheim, Wi: Wilmersdorf, Da: Darzau 
Tab. 8: Summe der monatlichen Niederschläge $(\mathrm{mm})$ über die Vegetationsperioden 2004 bis 2006 an den vier Standorten

\begin{tabular}{|c|c|c|c|c|c|c|c|c|c|c|}
\hline \multirow[b]{2}{*}{ Monat } & \multicolumn{3}{|c|}{2004} & \multicolumn{3}{|c|}{2005} & \multicolumn{4}{|c|}{2006} \\
\hline & Gö & Ho & $\mathbf{W i}$ & Gö & Ho & $\mathbf{W i}$ & Gö & Ho & $\mathbf{W i}$ & $\mathrm{Da}$ \\
\hline März & 27 & 37 & 30 & 27 & 52 & 15 & 63 & 80 & 30 & 63 \\
\hline April & 33 & 26 & 27 & 34 & 61 & 10 & 58 & 97 & 27 & 59 \\
\hline Mai & 88 & 73 & 43 & 83 & 86 & 72 & 97 & 68 & 43 & 50 \\
\hline Juni & 63 & 50 & 37 & 50 & 48 & 21 & 95 & 91 & 47 & 25 \\
\hline Juli & 93 & 62 & 29 & 70 & 127 & 78 & 83 & 63 & 29 & 23 \\
\hline Aug. & 85 & 86 & 104 & 63 & 128 & 44 & 101 & 172 & 104 & 92 \\
\hline Sept. & 48 & 47 & 25 & 35 & 36 & 34 & 13 & 35 & 25 & 16 \\
\hline Summe & 437 & 381 & 295 & 362 & 538 & 274 & 510 & 607 & 305 & 329 \\
\hline
\end{tabular}

Gö: Göttingen, Ho: Hohenheim, Wi: Wilmersdorf, Da: Darzau

\subsubsection{Ausleseverfahren}

\section{Stammbaummethode}

In den ersten beiden Jahren fand eine Auslese von Linien in Göttingen statt. Die vergleichende Prüfung dieser Linien wurde in 2006 an vier Standorten durchgeführt.

In 2004 wurden pro Kreuzung 50 F3-Pflanzen in ein Meter langen Doppelreihen in Göttingen angebaut. Pro Doppelreihe wurden 20 Achänen am 7. April 2004 ausgesät. Die Ernte fand vom 13. bis 15. September statt. Die Vorfrucht war Winterweizen mit der Zwischenfrucht Senf. Folgende Merkmale wurden auf dem Feld erfasst:

- Blühbeginn (numerischer Tag bei 5\% blühenden Pflanzen)

- Blühende (numerischer Tag bei $75 \%$ abgeblühter Pflanzen)

- Reifezeitpunkt (numerischer Tag bei 75\% reifen Pflanzen)

- Pflanzenlänge zu Blühbeginn (cm)

- Lager (1: kein Lager, 9: starkes Lager)

- Köpfchenfäule (1: kein Befall, 9: sehr starker Befall)

- Alternaria-Blattflecken (1: kein Befall, 9: sehr starker Befall). 
Anhand dieser Daten über Standfestigkeit und Krankheitsbefall wurde eine erste Selektion vor der Ernte durchgeführt. Die Termine der Bonitur sind im Anhang Tab. A I zu finden. Insgesamt wurden 35 F3-Linien in der ersten, 34 in der zweiten und 40 in der dritten Kreuzung einzelpflanzenweise geerntet. Pro F3-Linie wurden zehn F3-Pflanzen zufällig ausgewählt.

Die Ernteproben wurden als intakte Achänen anschließend mit der NIRSKalibration Ölgehalt 1 auf ihren Ölgehalt untersucht. Zunächst wurde der Ölgehalt aller Einzelpflanzen bestimmt und anschließend der Durchschnitt des Ölgehaltes pro Linie errechnet. In den besten 14 F3-Linien pro Kreuzung wurden sieben Einzelpflanzen zufällig für den Anbau in 2005 ausgewählt. Diese sieben Einzelpflanzen stammten von einer F2-Pflanze ab und bildeten zusammen eine F2:4-Familie. Die Daten der Merkmale Lager, Köpfchenfäule, Alternaria-Blattflecken und Ölgehalt der F3-Linien sind im Anhang Tab. A V Tab. A VII zu finden.

In 2005 wurden von den 98 selektierten Pflanzen je Kreuzung je 40 Achänen in ein Meter langen Doppelreihen am 18. April ausgesät. Die Vorfrucht war Sommerweizen mit einer Blanksaat Rotkleegras. Um Auskreuzung zwischen den Kreuzungen zu verhindern, wurden Ränder zwischen den einzelnen Kreuzungen angebaut. Die Ränder bestanden jeweils aus einer Mischung von Pflanzen der gleichen Kreuzung, an die der Rand angrenzte.

Es wurden die gleichen Merkmale wie 2004 bonitiert. Vor der Ernte (12. bis 14. September) fand jedoch keine Selektion statt. Um genügend Saatgut für die Parzellenaussaat 2006 an vier Standorten zu gewinnen, wurden jeweils alle Einzelpflanzen einer Doppelreihe zusammen geerntet. $\mathrm{Da}$ in manchen Doppelreihen keine Pflanzen aufgelaufen waren, wurden 91 (Kreuzung 1), 97 (Kreuzung 2) und 72 (Kreuzung 3) F4-Linien geerntet. Mittels NIRS wurde anhand der Kalibration Ölgehalt 2 der Ölgehalt des Erntegutes bestimmt.

Die Selektion der besten F4-Linien erfolgte auf der Grundlage von Krankheitsbefall (Alternaria-Blattflecken und Köpfchenfäule) und Ölgehalt. Eine Varianzanalyse mit dem Programm PLABSTAT (Version 2N(L), 1997) zeigte, dass die Varianz in diesen Merkmalen zwischen den 14 F2:4-Familien in allen drei Kreuzungen signifikant größer war als die innerhalb der sieben F4-Linien einer Familie (Anhang Tab. A II). Es wurden pro Kreuzung diejenigen Familien ausgewählt, die in zwei der drei Merkmale über dem Durchschnitt lagen. In den 
Kreuzungen 1 und 3 waren dies acht Familien, in der Kreuzung 2 sieben (Anhang Tab. A IX - Tab. A XI). Nur von sieben F2:4-Familien pro Kreuzung war genügend Saatgut vorhanden. Innerhalb dieser F2:4-Familien wurden die vier besten F4-Linien nach ihrem Ölgehalt ausgewählt. In den Kreuzungen 1 und 2 wurde je eine F4-Linie zusätzlich und in der Kreuzung 3 eine F4-Linie weniger ausgewählt.

In 2006 fand die vergleichende Prüfung der Linien an vier Standorten statt. In den Kreuzungen 1 und 2 wurden je 29 F5-Linien angebaut und in der Kreuzung 3 waren es 27 F5-Linien (Abb. 7). 


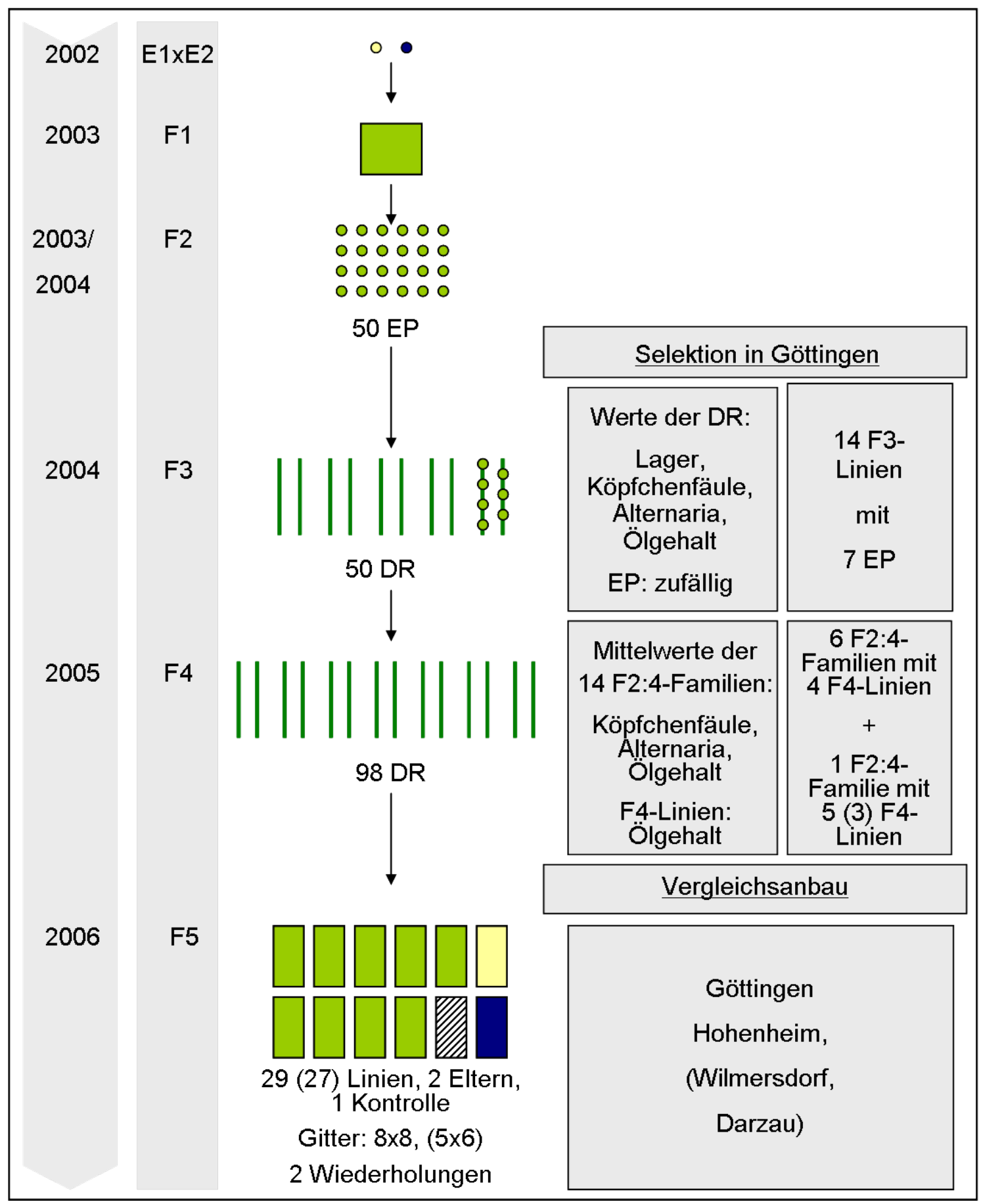

EP: Einzelpflanze, DR: Doppelreihe

Abb. 7: Schema der Stammbaummethode

Da 2005 sehr unterschiedlicher Aufgang beobachtet worden war, wurde vor der Aussaat im Frühjahr 2006 von jedem Prüfglied die Keimfähigkeit und das TKG (Tausendkorngewicht, errechnet aus dem Gewicht von zwei mal 100 Achänen) bestimmt und die Aussaatmenge jeder Parzelle auf 40 keimfähige Achänen pro Quadratmeter errechnet. 
Die Kreuzungen 1 und 2 wurden an den oben genannten vier Standorten in einem 8x8 Gitter mit zwei Wiederholungen geprüft. Zusätzlich wurden in die Versuchsanlage die beiden Eltern der jeweiligen Kreuzung und pro Kreuzung eine Kontrolle integriert. Die Kreuzung 3 wurde in einem 5x6 Gitter mit zwei Wiederholungen an nur zwei Standorten (Göttingen und Hohenheim) ebenfalls zusammen mit einer Kontrolle und den beiden Eltern (Sabina und AC Sunset) angebaut.

Die Kontrolle bestand aus einer Mischung von unselektierten F7-Pflanzen aus dem Gewächshaus. Die 50 F2-Pflanzen pro Kreuzung wurden im Gewächshaus bis zur F6 weitergeführt (Einkornramschmethode). Je vier Achänen jeder der 50 F6-Pflanzen pro Kreuzung wurden abgezählt und gemischt, sodass für jede Kreuzung eine unselektierte Kontrolle entstand.

Die auf dem Feld erfassten Merkmale waren die gleichen wie 2004. AlternariaBlattflecken traten nur in Göttingen und Hohenheim differenziert auf. Zusätzlich wurde Fusarium beobachtet. Die Termine der Bonitur finden sich im Anhang Tab. A I. Die Anzahl der mit Fusarium befallenen Pflanzen wurde in Prozent zu der gesamten Anzahl Pflanzen in einer Parzelle erfasst.

Nach der Ernte wurden folgende Merkmale untersucht:

- Ölgehalt (\%), mittels NIRS-Kalibration Ölgehalt 3

- bereinigter Einzelpflanzenertrag (g), siehe unten

- $\quad$ TKG (g), wie 2004

- Linolsäure- und Ölsäurehalt (\%), mittels NIRS

- Schalenanteil (\%), mittels NIRS

Der Feldaufgang fiel sehr unterschiedlich aus, sodass der Ertrag der Parzellen untereinander nicht vergleichbar war. Es wurde eine Abhängigkeit des Einzelpflanzenertrages von der Anzahl Pflanzen in einer Parzelle festgestellt. Deshalb wurde der Ertrag als bereinigter Einzelpflanzenertrag (im folgenden EP-Ertrag genannt) erfasst. Zunächst fand die Berechnung einer Regression von dem Einzelpflanzenertrag auf die Anzahl Pflanzen statt. Anschließend wurde für jeden Standort der bereinigte Einzelpflanzenertrag berechnet, der von der Anzahl Pflanzen in einer Parzelle unabhängig ist: 


$$
\begin{array}{ll}
y^{\prime}=y+(\bar{x}-x) \cdot r, \text { wobei } \\
y^{\prime} & : \text { bereinigter Einzelpflanzenertrag } \\
y & : \text { Einzelpflanzenertrag } \\
\bar{x} & : \text { Mittel der Anzahl Pflanzen in einer Kreuzung } \\
x & : \text { Anzahl Pflanzen in der Parzelle } \\
r & : \text { Regressionskoeffizient (Regression von y auf } x)
\end{array}
$$

Die Werte der phänologischen Daten (Blühbeginn, Blühende, Blühdauer und Reife), der Pflanzenlänge, des Linolsäure- und Ölsäuregehaltes sind im Anhang Tab. A XII und Tab. A XIII zu finden.

Die Parzellengröße betrug in Göttingen und Hohenheim jeweils 4,2 $\mathrm{m}^{2}$ und in Wilmersdorf und Darzau 5,04 $\mathrm{m}^{2}$. Ausgesät wurde in Göttingen und Hohenheim am 20. April, in Wilmersdorf und Darzau am 21. April. Die Ernte fand am 5. September in Göttingen, am 6. September in Hohenheim und Wilmersdorf und am 24. August in Darzau statt. In Hohenheim wurden aufgrund von schlechtem Feldaufgang nur fünf gut entwickelte Pflanzen pro Parzelle geerntet. Daher sind die Ertragswerte mit den anderen Orten nicht direkt vergleichbar.

Als Vorfrüchte wurden in Göttingen Erbsen, in Wilmersdorf Lupinen angebaut und in Hohenheim wuchs im Vorjahr Hafer, in Darzau Winterweizen.

Die Standorte wurden einzeln mit dem Programm PLABSTAT (Version 2N(L), 1997) als Gitteranlage verrechnet (Utz 2001). Anschließend wurden die ermittelten Prüfgliedmittelwerte weiteren Varianzanalysen über die Standorte unterzogen. Zur Aufteilung der Prüfgliedvarianz wurden für die Daten der Linien plus beiden Eltern und Kontrolle, für die Daten der Linien und beiden Eltern, für die Daten der Linien plus Kontrolle, für die Daten der Linien, für die Daten beider Eltern und Kontrolle und für die Daten der beiden Eltern jeweils getrennte Varianzanalysen durchgeführt. 
Das Modell lautete für die mehrortige Verrechnung:

$$
\begin{array}{ll}
\mathrm{Y}_{\mathrm{ij}}=\mu+\mathrm{G}_{\mathrm{i}}+\mathrm{O}_{\mathrm{j}}+\mathrm{GO}_{\mathrm{ij}}+\mathrm{e}_{\mathrm{ij}}, \text { wobei } \\
\mathrm{Y}_{\mathrm{ij}} & : \text { Beobachtungswert des Genotyps i am Standort j } \\
\mu & : \text { allgemeiner Mittelwert, } \\
\mathrm{G}_{\mathrm{i}} & : \text { Effekt des Genotyps i (Linien, Eltern und Kontrolle), } \\
\mathrm{O}_{\mathrm{j}} & : \text { Effekt des Standortes j } \\
\mathrm{GO}_{\mathrm{ij}} & : \text { Interaktion zwischen den Genotyp i und den Standort j } \\
\mathrm{e}_{\mathrm{ij}} & : \text { Fehler }
\end{array}
$$

In der Varianzanalyse wurde getestet, ob es signifikante Unterschiede zwischen den Linien und dem Mittel der beiden Eltern plus der Kontrolle (später mit L vs $(\mathrm{K}+2 \mathrm{E})$ bezeichnet), zwischen der Kontrolle und dem Mittel der beiden Eltern ( $\mathrm{K}$ vs 2E), zwischen den beiden Eltern (E1 vs E2) und zwischen den 29 (27) Linien (L) gab. Zusätzliche Untersuchungen galten den Genotyp-Umweltinteraktionen (GxO). Im F-Test wurde gegen die Interaktion getestet.

\section{Natürliche Selektion}

Für die natürliche Selektion wurden 2004 pro Kreuzung von jeder der 50 F2Pflanzen, die auch Ausgangsmaterial für die Stammbaummethode waren, sieben Achänen gemischt. Jede Kreuzung wurde an drei Standorten (Göttingen, Hohenheim und Wilmersdorf) für den ersten Zyklus der natürlichen Auslese angebaut.

Für eine weitere Generation natürlicher Auslese wurde 2005 eine Stichprobe des Erntegutes jeden Ortes (Herkunftsort) aus 2004 an demselben Standort als F4-Parzelle erneut angebaut. Um einen gleichmäßigen Aufgang zu erhalten, wurde die Keimfähigkeit getestet und anschließend die Aussaatmenge für jede Parzelle einzeln berechnet. Um Auskreuzung zwischen den Parzellen zu verhindern (vergleiche Kapitel 4) waren in Göttingen und Hohenheim die Parzellen in isolierten Lagen angelegt. In Wilmersdorf konnte dies aus 
technischen Gründen nicht geschehen. Die Aussaat- und Erntetermine, sowie die Aussaatstärke, Parzellengröße und Vorfrucht sind der Tab. $9 \mathrm{zu}$ entnehmen. In Hohenheim gab es in den Parzellen der Kreuzung 1 und 3 Fraßschäden von Hasen, sodass dort weniger Saatgut geerntet wurde. Die Keimfähigkeit des Erntegutes wurde wie in 2004 bestimmt.

Tab. 9: Aussaatparameter, Erntetermin und Vorfrucht der Versuchsstandorte

\begin{tabular}{|c|c|c|c|c|c|}
\hline & $\begin{array}{c}\text { Aussaat- } \\
\text { termin }\end{array}$ & $\begin{array}{c}\text { Aussaatdichte } \\
\text { (keimfähige } \\
\text { Achänen } / \mathbf{m}^{2} \text { ) }\end{array}$ & $\begin{array}{l}\text { Parzellen- } \\
\text { größe }\left(m^{2}\right)\end{array}$ & $\begin{array}{l}\text { Ernte- } \\
\text { termin }\end{array}$ & Vorfrucht ${ }^{a}$ \\
\hline \multicolumn{6}{|l|}{2004} \\
\hline Göttingen & 30.03 .04 & 35 & 10,0 & 15.09 .04 & WW \\
\hline Hohenheim & 30.03 .04 & 35 & 10,0 & 07.09.04 & Dinkel \\
\hline Wilmersdorf & 01.04 .04 & 35 & 10,0 & 20.09 .04 & Kleegras \\
\hline \multicolumn{6}{|l|}{2005} \\
\hline \multirow[t]{2}{*}{ Göttingen } & 18.04 .05 & 40 & 18,0 & 14.09 .05 & Triticale (Kr. 1) \\
\hline & & & & & WR (Kr. 3) \\
\hline \multirow[t]{3}{*}{ Hohenheim } & 14.04 .05 & 40 & 12,0 & 13.09 .05 & $\begin{array}{l}\text { WR (Kr. 3) } \\
\text { Mais (Kr. 1) }\end{array}$ \\
\hline & & & & & Kleegras (Kr. 2) \\
\hline & & & & & Triticale (Kr.3) \\
\hline $\begin{array}{l}\text { Wilmersdorf } \\
2006\end{array}$ & 29.03 .05 & 40 & 12,0 & 20.09 .05 & Kleegras \\
\hline Göttingen & 20.04 .06 & 40 & 4,2 & 05.09 .06 & Erbsen \\
\hline Hohenheim & 20.04 .06 & 40 & 4,2 & 06.09 .05 & Hafer \\
\hline Wilmersdorf & 21.04 .06 & 40 & 5,0 & 06.09 .06 & Lupinen \\
\hline Darzau & 21.04 .06 & 40 & 5,0 & 24.08 .06 & WW \\
\hline
\end{tabular}

a WW: Winterweizen, WR: Winterroggen

In 2006 fand der Vergleichsanbau statt. Das Erntegut der drei Kreuzungen aller Herkunftsorte aus 2005 wurde an vier Standorten (Göttingen, Hohenheim, Wilmersdorf und Darzau) in einer Spaltanlage in zweifacher Wiederholung angebaut (vergleiche Tab. 9).

Die Großteilstücke beinhalteten die Kreuzungen, die Kleinteilstücke die einzelnen Herkünfte und die für die Kreuzung jeweilige Kontrolle, wie sie bereits im Rahmen der Stammbaummethode beschrieben wurde. Die Prüfglieder werden folgendermaßen benannt: nach einem $R$ (Ramschparzelle) folgen die Nummer der Kreuzung (1, 2, oder 3) und die Abkürzung des Herkunftsortes (Gö, Ho oder Wi). Handelt es sich um den Mittelwert über die drei Kreuzungen eines Herkunftsortes, so werden sie als R Gö, R Ho oder R Wi bezeichnet. 
Das Erntegut aus 2005 der Parzellen R1 Ho und R3 Ho wies eine sehr geringe Keimfähigkeit auf, sodass hier das Erntegut aus dem Jahr 2004 verwendet werden musste. Auf dem Feld wurden die gleichen Merkmale wie in der Stammbaumethode erfasst. Nach der Ernte wurden der Ölgehalt, der bereinigte Einzelpflanzenertrag (genannt EP-Ertrag, siehe Stammbaummethode) und das TKG ermittelt.

Die statistischen Berechnungen der Spaltanlage wurden mit dem Programm PLABSTAT (Version 2N(L), 1997) durchgeführt (Utz 2001). Die Wiederholungen wurden als zufällig angenommen.

Das Model der Varianzanalyse lautete:

$$
\begin{aligned}
& \mathrm{Y}_{\mathrm{ijlm}}=\mu+\mathrm{O}_{\mathrm{i}}+\mathrm{C}_{\mathrm{j}}+\mathrm{W}_{\mathrm{il}}+\mathrm{OC}_{\mathrm{ij}}+\mathrm{e}_{\mathrm{ijl}}+\mathrm{H}_{\mathrm{m}}+\mathrm{CH}_{\mathrm{jm}}+\mathrm{OH}_{\mathrm{im}}+\mathrm{OCH}_{\mathrm{ijm}}+\mathrm{e}_{\mathrm{ijml}} \text {, wobei } \\
& \mathrm{Y}_{\mathrm{ijlm}} \quad \text { : Beobachtungswert am Standort } \mathrm{i} \text { von Kreuzung } \mathrm{j} \text { in Wiederholung I } \\
& \text { von Herkunftsort m } \\
& \mu \quad \text { : allgemeiner Mittelwert } \\
& \mathrm{O}_{\mathrm{i}} \quad \text { : Haupteffekt des Standortes } \mathrm{i} \\
& C_{j} \quad: \text { Haupteffekt der Kreuzung j (Großteilstückfaktor) } \\
& \mathrm{W}_{\mathrm{il}} \quad \text { : Effekt der Wiederholung I innerhalb des Standortes } \mathrm{i} \\
& \mathrm{OC}_{\mathrm{ij}} \quad \text { : Interaktion zwischen Standort i und Kreuzung j } \\
& \mathrm{e}_{\mathrm{ijl}} \quad \text { : Fehler der Großteilstücke } \\
& \mathrm{H}_{\mathrm{m}} \quad \text { : Haupteffekt des Herkunftsortes m (Kleinteilstück) } \\
& \mathrm{CH}_{\mathrm{jm}} \quad \text { : Interaktion zwischen Kreuzung j und Herkunftsort } \mathrm{m} \\
& \mathrm{OH}_{\mathrm{im}} \quad \text { : Interaktion zwischen Standort i und Herkunftsort } \mathrm{m} \\
& \mathrm{OCH}_{\mathrm{ijm}} \quad \text { : Interaktion zwischen Standort i, Kreuzung j und Herkunftsort m } \\
& \mathrm{e}_{\mathrm{ijml}} \quad \text { : Fehler der Kleinteilstücke }
\end{aligned}
$$




\section{Einkornramsch}

Die Grundlage der Einkornramschmethode bildeten die 50 F2-Pflanzen pro Kreuzung, die bereits im Rahmen der Stammbaummethode und der natürlichen Selektion erwähnt wurden. Im Frühjahr 2004 wurde je ein Nachkomme der 150 F2-Pflanzen ausgesät. Die Pflanzen wuchsen im Gewächshaus, sodass bereits im Herbst 2004 je ein Nachkomme der F3-Pflanzen angebaut werden konnte. Pro Jahr wurden zwei Generationen einzelpflanzenweise weitergeführt, sodass im Herbst 2006 die F6-Pflanzen ausgesät wurden. Hier wurden pro F5-Pflanze zwei Achänen ausgelegt. Das Erntegut der zusätzlichen F6-Pflanzen wurde pro Kreuzung zu gleichen Teilen gemischt und als unselektierte Kontrolle in die Versuchsanlagen der Stammbaummethode und der natürlichen Selektion integriert.

Die F7-Prüfglieder wurden 2006 an zwei Orten (Göttingen und Hohenheim) zusammen mit den Eltern in ein Meter langen Doppelreihen angebaut. Je 20 Achänen pro Doppelreihe wurden ausgesät. Als Hilfe für die Bonituren wurde die Sorte Sabina in regelmäßigen Abständen als Standard in die Versuchsanlage eingefügt. Aufgrund der begrenzten Menge an Saatgut, das von Einzelpflanzen geerntet wurde, konnten an den Orten keine Wiederholungen angelegt werden. Die erfassten Merkmale waren die gleichen wie in der Stammbaummethode. Der Ertrag pro Doppelreihe wurde gewogen und anschließend der bereinigte Einzelpflanzenertrag (EP-Ertrag, siehe Stammbaummethode) berechnet. Die Aussaat fand in Göttingen, wo im Vorjahr Erbsen standen, am 24. April statt, geerntet wurde am 5. September. In Hohenheim wurde am 21. April nach der Vorfrucht Hafer ausgesät. Der Aufgang in Hohenheim war aus ungeklärten Gründen sehr schlecht. Deshalb wurden nur die Daten aus Göttingen ausgewertet.

Die statistische Analyse des Versuches wurde mit dem Programm PLABSTAT (Version 3Awin-mini, 2005) durchgeführt. Die Adjustierung der Parzellen wurde für jede Kreuzung über gleitende Mittelwerte berechnet. Die Daten der Standards wurden nicht mit verrechnet. Die Mittelwerte der direkt angrenzenden Parzellen wurden als Kovariable zusammengefasst und für die Korrektur verwendet (Utz 2005). 


\subsection{Ergebnisse}

\subsubsection{Stammbaummethode}

\section{Durchführung der Auslese}

Die Mittelwerte der Linien und die Daten der Eltern für die phänologischen Merkmale (Blühbeginn, Blühende, Blühdauer und Reife) und die Pflanzenlänge 2004 und 2005 finden sich im Anhang Tab. A III. Die Daten der im Folgenden beschriebenen Merkmale (Lager, Köpfchenfäule, Alternaria-Blattflecken und Ölgehalt) der Eltern und der F3-Linien aus 2004 sind im Anhang Tab. A IV Tab. A VII dargestellt. Ebenfalls im Anhang finden sich die Daten der Eltern und der F2:4-Familien aus 2005 (Tab. A VIII - Tab. A XI).

In 2004 wurde für die Selektion das Merkmal Lager der F3-Pflanzen in den Doppelreihen berücksichtigt. In der Kreuzung 1 wurden acht Doppelreihen (Boniturnote größer als 4), in Kreuzung 2 sechs Doppelreihen (Boniturnote größer als 5) und in der Kreuzung 3 zwei Doppelreihen (Boniturnote größer als 4) nicht weitergeführt. In allen Kreuzungen war eine große Variation im Merkmal Lager zu erkennen (Tab. 10).

Tab. 10: Boniturergebnisse für das Merkmal Lager (Note) in Göttingen 2004

\begin{tabular}{lccc}
\hline & \multicolumn{3}{c}{ Lager } \\
\cline { 2 - 4 } Kreuzung & Mittel & Minimum & Maximum \\
\hline Kreuzung 1 & 3,14 & 2,0 & 7,0 \\
Kreuzung 2 & 3,96 & 2,0 & 7,0 \\
Kreuzung 3 & 3,20 & 2,0 & 5,0 \\
\hline
\end{tabular}

Sowohl 2004 als auch 2005 wurde der Befall mit Alternaria-Blattflecken und Köpfchenfäule für die Selektion herangezogen.

Die Köpfchenfäule trat 2005 insgesamt stärker auf als 2004. Besonders betroffen war der Elter AC Sunset. CR1 und Sabina zeigten den geringsten Befall (Abb. 8). In 2004 wurden aufgrund von Köpfchenfäule sieben F3-Linien in der Kreuzung 1 (Boniturnote größer als 2), neun F3-Linien in der Kreuzung 2 (Boniturnote größer als 2) und neun F3-Linien in der Kreuzung 3 (Boniturnote größer als 1) verworfen. In 2005 lagen in der Kreuzung 1 acht F2:4-Familien über dem Durchschnitt, in der Kreuzung 2 sieben F2:4-Familien und neun F2:4Familien in der Kreuzung 3. 


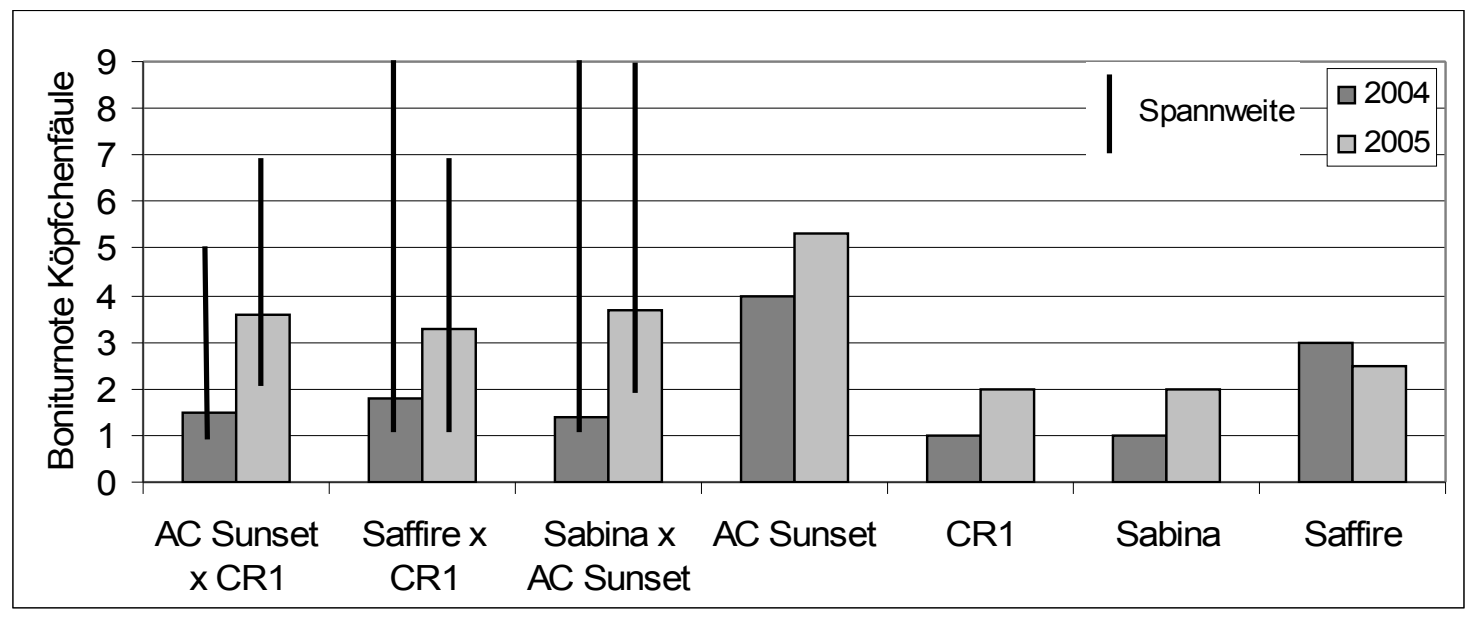

Abb. 8: Köpfchenfäule; Mittlere Boniturnoten von Kreuzungen und Eltern (2004: 50 F3-Linien pro Kreuzung, 2005: 91, 97 und 72 F4-Linien pro Kreuzung)

Der Befall mit Alternaria-Blattflecken war in den Kreuzungen 2005 stärker (Abb. 9). Wie in der Literatur beschrieben, zeigte Saffire in beiden Jahren und AC Sunset in 2004 eine hohe Anfälligkeit (Mündel et al. 2004). Der geringe Befall 2005 von AC Sunset war nicht zu erwarten. Möglicherweise überdeckte der hohe Köpfchenfäulebefall die Symptome der Alternaria-Blattflecken.

In 2004 wurden in den Kreuzungen 1 und 3 jeweils zwei F3-Linien (Boniturnote größer 3 ) und in der Kreuzung 2 ebenfalls zwei F3-Linien (Boniturnote größer 5) nicht weitergeführt. In 2005 lagen neun F2:4-Familien in der ersten und jeweils acht in der zweiten und dritten Kreuzung über dem Durchschnitt.

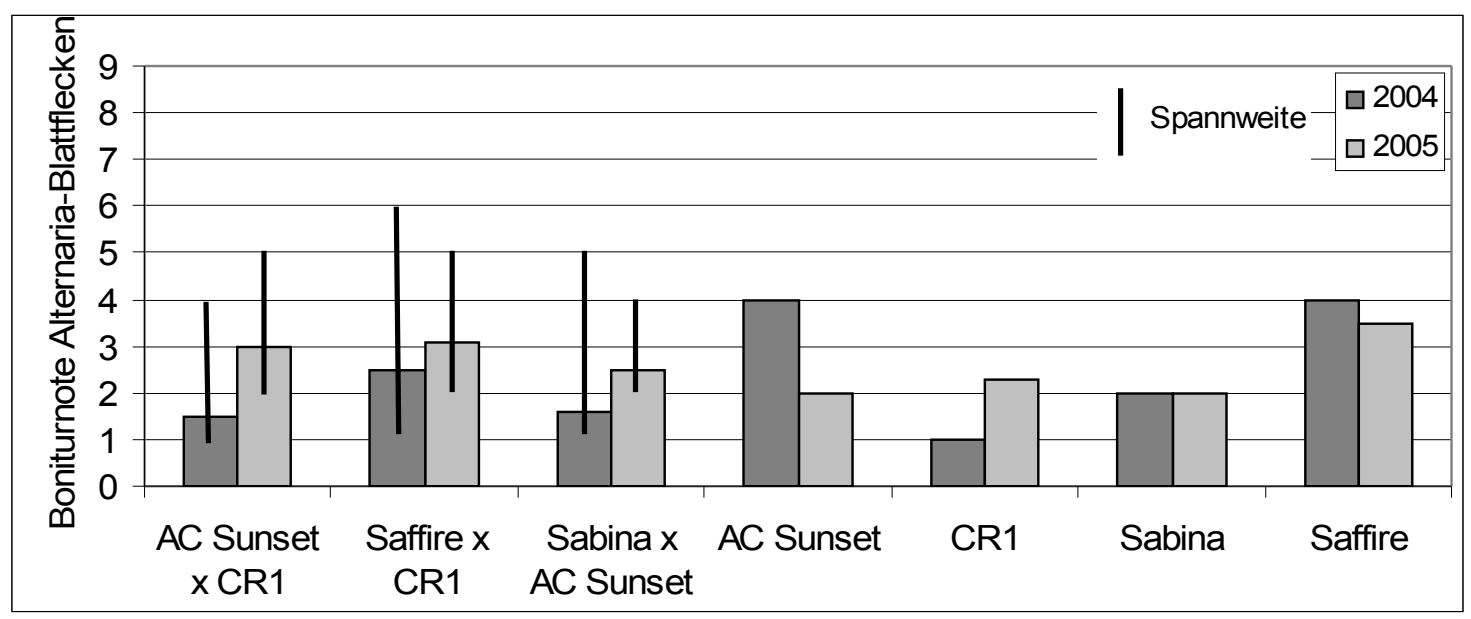

Abb. 9: Alternaria-Blattflecken; Mittlere Boniturnoten von Kreuzungen und Eltern (2004: 50 F3-Linien pro Kreuzung, 2005: 91, 97 und 72 F4Linien pro Kreuzung) 
Der durchschnittliche Ölgehalt pro Kreuzung war 2004 und 2005 ähnlich. In allen drei Kreuzungen war eine große Variation zu erkennen. In Abb. 10 sind für jede Kreuzung die Spannweiten des Ölgehaltes der F3- und F4-Linien dargestellt. Nicht eingezeichnet sind die Spannweiten bezogen auf den Ölgehalt der F3-Einzelpflanzen in 2004: Kreuzung 1: 10,2 bis 29,1\%, Kreuzung 2: 7,6 bis $29,4 \%$, Kreuzung 3: 3,5 bis $28,2 \%$. Von den Eltern erreichte Saffire mit 23,2\% (2004) und 24,5\% (2005) den höchsten Ölgehalt.

In 2004 wurde der Ölgehalt der F3-Linien für die Selektion verwendet. In den jeweils besten 14 Doppelreihen wurden sieben Pflanzen zufällig für den Anbau 2005 ausgewählt. In 2005 lagen in der ersten Kreuzung sechs, in der zweiten und in der dritten Kreuzung jeweils acht F2:4-Familien über dem Durchschnitt.

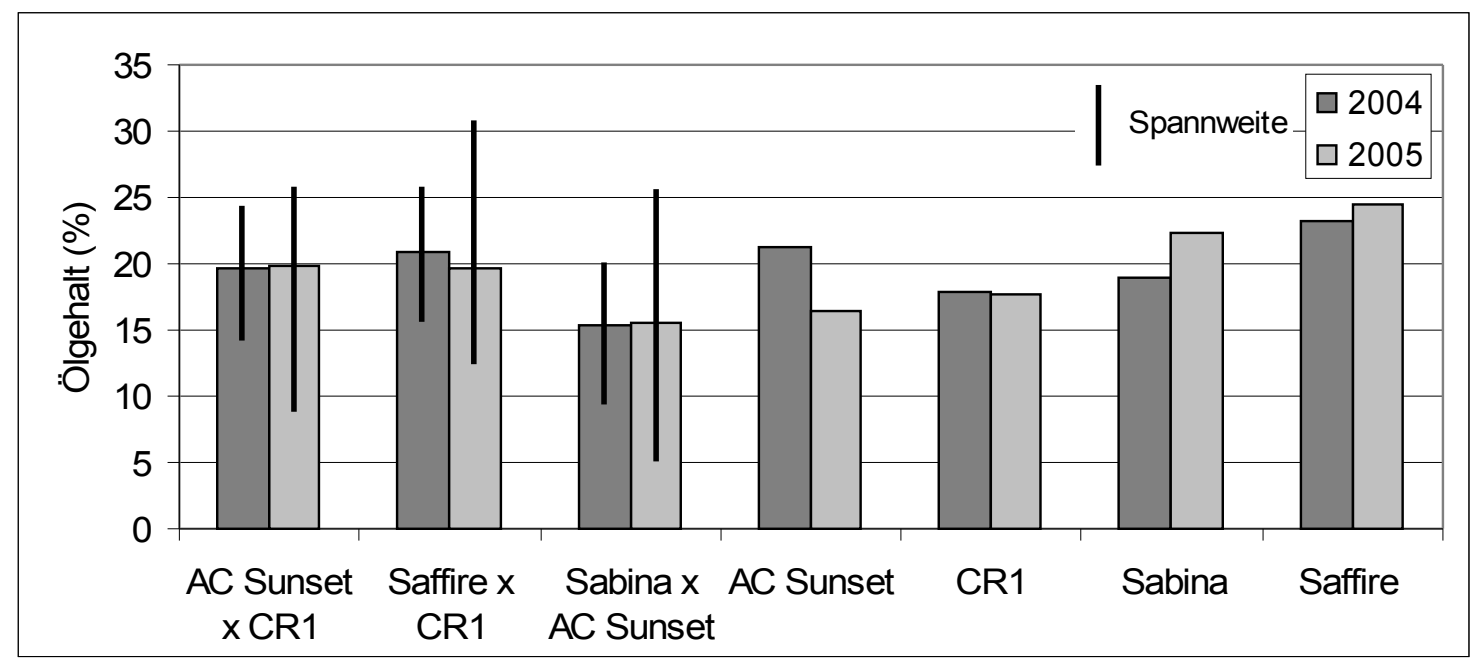

Abb. 10: Durchschnittlicher Ölgehalt (\%) 2004 und 2005 (2004: 35, 34 und 40 F3-Linien pro Kreuzung, 2005: 91, 97 und 72 F4-Linien pro Kreuzung)

In 2005 wurden sieben F2:4-Linien ausgewählt, die in zwei der drei Merkmale (Köpfchenfäule, Alternaria-Blattflecken und Ölgehalt) über dem Durchschnitt der F2:4-Familien lagen (Anhang Tab. A IX - Tab. A XI). In jeder Familie fand eine Auswahl von vier F4-Linien nach ihrem Ölgehalt statt. In den Kreuzungen 1 und 2 wurde eine F4-Linie zusätzlich und in der Kreuzung 3 eine F4-Linie weniger ausgewählt. 


\section{Ergebnisse des Vergleichsanbaus}

Die jeweils 29 besten F5-Linien der Kreuzung 1 und 2 wurden 2006 an vier Orten (Göttingen, Hohenheim, Wilmersdorf und Darzau), die besten 27 Linien der Kreuzung 3 an zwei Orten (Göttingen und Hohenheim) angebaut. Zusätzlich waren in den Versuchsanlagen die Eltern und die nicht selektierte Kontrolle für jede einzelne Kreuzung integriert. Der Feldbestand war an den Standorten sehr unterschiedlich. In Göttingen standen im Mittel 14,7 Pflanzen pro $\mathrm{m}^{2}$ (Minimum: 7,4, Maximum: 30,9) in Hohenheim 5,1 (Minimum: 0,5, Maximum: 21,9), in Wilmersdorf 10,4 (Minimum: 2,4, Maximum: 22,8) und in Darzau 8,8 (Minimum: 3,2, Maximum: 15,5).

In der Varianzanalyse wurden die 29 Linien (27 in Kreuzung 3) gegen die Kontrolle und die beiden Eltern $(L$ vs $(K+2 E))$ und die Kontrolle gegen das Mittel der beiden Eltern (K vs $2 \mathrm{E}$ ) getestet. Außerdem wurde untersucht, ob sich die beiden Eltern (E1 vs E2) und ob sich die 29 (27) Linien signifikant unterschieden. Zudem wurde die Interaktion (GxO) zwischen den 32 (30 in Kreuzung 3) Genotypen (G) und den Orten (O) getestet.

\section{Köpfchenfäule}

Die Linien waren in der ersten Kreuzung signifikant vom Mittel aus Kontrolle und Eltern verschieden. Die beiden Eltern der ersten Kreuzung (AC Sunset und CR1) unterschieden sich hoch signifikant und die beiden Eltern der dritten Kreuzung (Sabina und AC Sunset) signifikant voneinander. Eine Interaktion zwischen den Genotypen und den Orten zeigte sich in der ersten und dritten Kreuzung (siehe Tab. 11).

Tab. 11: Ergebnisse der Varianzanalyse für das Merkmal Köpfchenfäule (Note) an vier (Kreuzung 3: zwei) Standorten 2006

\begin{tabular}{|c|c|c|c|c|c|c|c|c|c|c|}
\hline \multirow[b]{2}{*}{ Varianzursache $^{a}$} & \multicolumn{4}{|c|}{ Kreuzung 1} & \multicolumn{3}{|c|}{ Kreuzung 2} & \multicolumn{3}{|c|}{ Kreuzung 3} \\
\hline & FG & MQ & F-We & & FG & MQ & F-Wert & FG & MQ & F-Wert \\
\hline $\bar{G}$ & 31 & 0,47 & 1,29 & & 31 & 0,35 & 1,25 & 29 & 0,70 & 0,63 \\
\hline L vs $(\mathrm{K}+2 \mathrm{E})$ & 1 & 1,72 & 4,67 & * & 1 & 0,74 & 2,63 & 1 & 0,05 & 0,04 \\
\hline $\mathrm{K}$ vs $2 \mathrm{E}$ & 1 & 0,01 & 0,01 & & 1 & 0,10 & 0,34 & 1 & 0,08 & 0,07 \\
\hline $\mathrm{E} 1$ vs E2 & 1 & 3,28 & 8,88 & ** & 1 & 0,14 & 0,49 & 1 & 7,70 & $6,90 *$ \\
\hline L & 28 & 0,35 & 0,94 & & 28 & 0,35 & 1,26 & 26 & 0,48 & 0,43 \\
\hline $\mathrm{GxO}$ & 93 & 0,37 & 1,39 & * & 93 & 0,28 & 1,06 & 29 & 1,12 & 4,48 ** \\
\hline Fehler & 196 & 0,27 & & & 196 & 0,27 & & 37 & 0,23 & \\
\hline
\end{tabular}

${ }^{* / *}$ signifikant bei $p=0,05 / 0,01,{ }^{a} \mathrm{G}$ : Genotypen, L: Linien, K: Kontrolle, E: Elter, O: Standorte 
In Abb. 11 sind die Boniturnoten der Genotypen für die drei Kreuzungen dargestellt. Der Elter 1 steht jeweils für die Mutter (Kreuzung 1: AC Sunset, Kreuzung 2: Saffire und Kreuzung 3: Sabina), der Elter 2 für den Vater (Kreuzung 1: CR1, Kreuzung 2: CR1 und Kreuzung 3: AC Sunset). Die Daten der Mittelwerte finden sich in der Tab. 12.

In allen drei Kreuzungen wiesen viele Linien einen geringeren Befall mit Köpfchenfäule auf als die unselektierte Kontrolle. In der ersten Kreuzung waren es 27 Linien, die besser waren als die Kontrolle, in der zweiten 23 und in der dritten 16. Signifikant besser waren in den ersten beiden Kreuzungen noch zwei Linien und in der dritten Kreuzung keine Linie. Es war in allen drei Kreuzungen ein Selektionserfolg (L-K) zu beobachten, besonders in den ersten beiden Kreuzungen waren die Linien im Mittel geringer befallen als die Kontrolle. Der Befall der Linien in Kreuzung 3 war relativ stark. In den Kreuzungen 1 und 2 war er geringer.

Der Elter AC Sunset war im Vergleich zu den anderen Eltern stark befallen. Die Boniturnoten der Linien variierten in der Kreuzung 1 zwischen 2,13 und 3,40, in der Kreuzung 2 zwischen 2,09 und 3,13. Große Variation zwischen 2,74 und 4,77 war in der Kreuzung 3 zu erkennen.
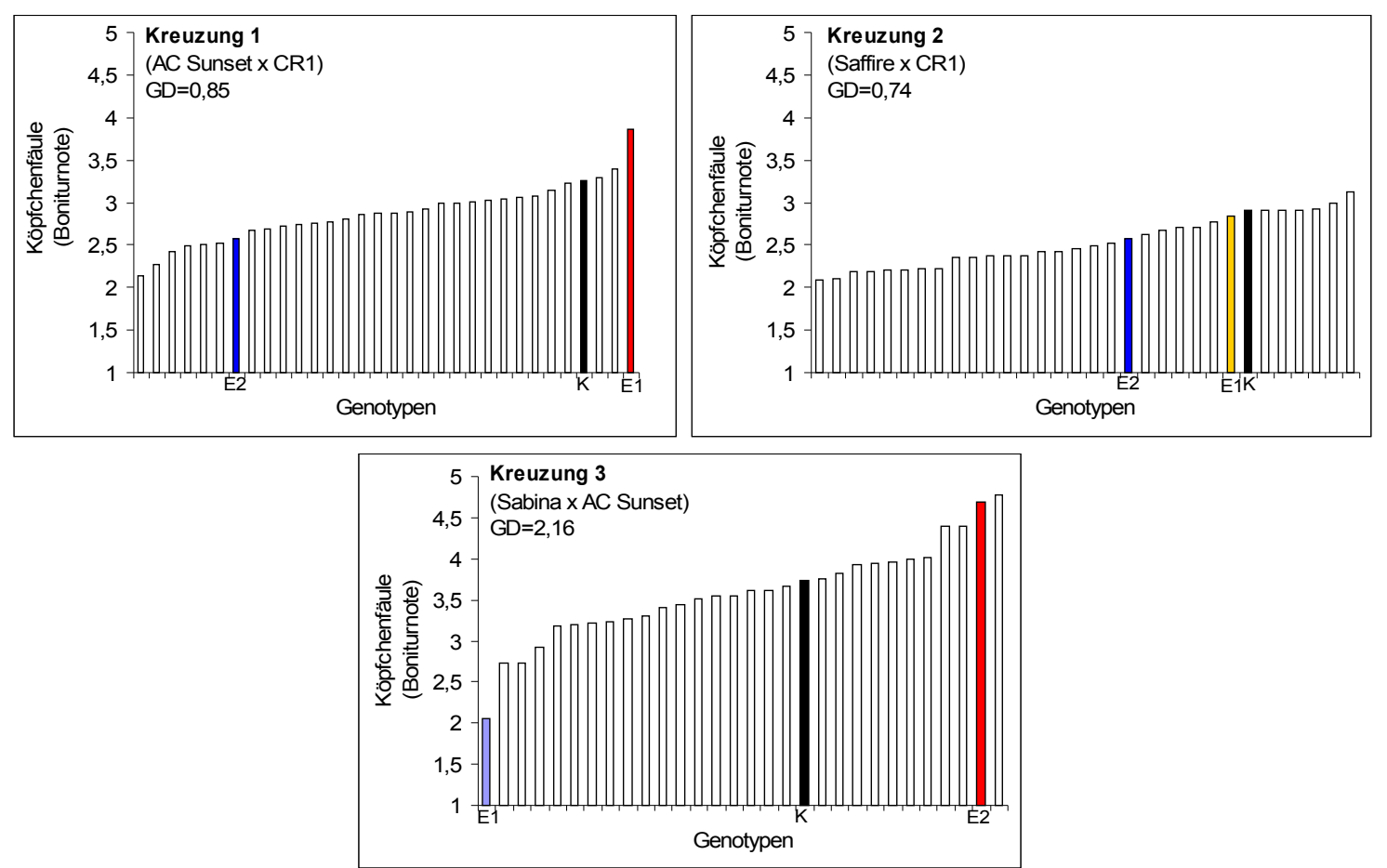

GD: Grenzdifferenz $(0,05)$

Abb. 11: Köpfchenfäule, Boniturnoten der 29 (27) Linien, der Kontrolle (K) und der Eltern (E1, E2), gemittelt über vier (zwei) Versuchsstandorte 
Um einen Überblick über die Verteilung der Daten zu erhalten, wurden zusätzlich die Ergebnisse der einzelnen Orte betrachtet (Tab. 12). Der Selektionserfolg war an den einzelnen Orten unterschiedlich. In Wilmersdorf und Darzau wirkte sich die Selektion in beiden Kreuzungen positiv aus. In Hohenheim dagegen war kein Erfolg der Selektion zu erkennen. Die Selektion war in Göttingen in den Kreuzungen 2 und 3 erfolgreich, in der ersten nicht. Der Befall war allgemein an den niederschlagsreicheren Standorten Göttingen und Hohenheim höher als in Wilmersdorf.

Tab. 12: Köpfchenfäule (Note), Befall an den Versuchsstandorten und im Mittel der Orte

\begin{tabular}{lccccc}
\hline & \multicolumn{5}{c}{ Standorte $^{\mathbf{a}}$} \\
\cline { 2 - 4 } & Gö & Ho & Wi & Da & Mittel \\
\hline Kreuzung 1 & & & & & \\
Elter 1 (AC Sunset) & 5,00 & 4,00 & 3,01 & 3,42 & 3,86 \\
Elter 2 (CR1) & 3,00 & 2,50 & 1,00 & 3,81 & 2,58 \\
Mittel der Linien (L) & 3,52 & 2,50 & 1,80 & 3,53 & 2,83 \\
Kontrolle (K) & 3,00 & 2,50 & 2,49 & 5,06 & 3,26 \\
L - K & 0,52 & 0,00 & $-0,69$ & $-1,53$ & $-0,43$ \\
Anzahl L besser als K & 0,00 & 7,00 & 24,00 & 27,00 & \\
Kreuzung 2 & & & & & \\
Elter 1 (Saffire) & 3,50 & 2,50 & 2,48 & 2,88 & 2,84 \\
Elter 2 (CR1) & 3,00 & 2,50 & 1,00 & 3,81 & 2,58 \\
Mittel der Linien (L) & 3,16 & 2,53 & 1,53 & 2,83 & 2,51 \\
Kontrolle (K) & 4,00 & 2,50 & 2,03 & 3,06 & 2,90 \\
L - K & $-0,84$ & 0,03 & $-0,50$ & $-0,23$ & $-0,39$ \\
Anzahl L besser als K & 27,00 & 7,00 & 23,00 & 20,00 & \\
Kreuzung 3 & & & & & \\
Elter 1 (Sabina) & 2,01 & 2,06 & & & 2,04 \\
Elter 2 (AC Sunset) & 4,93 & 4,69 & & & 3,81 \\
Mittel der Linien (L) & 3,30 & 3,90 & & & 3,60 \\
Kontrolle (K) & 3,56 & 3,78 & & $-0,07$ \\
L - K & $-0,26$ & 0,12 & & \\
Anzahl L besser als K & 17,00 & 11,00 & & & \\
\hline
\end{tabular}

a Gö: Göttingen, Ho: Hohenheim, Wi: Wilmersdorf, Da: Darzau

\section{Alternaria-Blattflecken}

In der dritten Kreuzung unterschieden sich die Linien signifikant vom Mittel der Kontrolle und den beiden Eltern. Die Eltern unterschieden sich in allen Kreuzungen hoch signifikant voneinander. Außerdem gab es in der Kreuzung 1 signifikante und in der Kreuzung 2 hoch signifikante Interaktionen zwischen den Genotypen und den Standorten (Tab. 13). 
Tab. 13: Ergebnisse der Varianzanalyse für das Merkmal AlternariaBlattflecken (Note) an zwei Orten 2006

\begin{tabular}{|c|c|c|c|c|c|c|c|c|c|c|}
\hline \multirow[b]{2}{*}{ Varianzursache $^{a}$} & \multicolumn{3}{|c|}{ Kreuzung 1} & \multicolumn{4}{|c|}{ Kreuzung 2} & \multicolumn{3}{|c|}{ Kreuzung 3} \\
\hline & FG & $\mathbf{M Q}$ & F-Wert & FG & MQ & F-We & & FG & $M Q$ & F-Wert \\
\hline$\overline{\mathrm{G}}$ & 31 & 0,57 & 1,51 & 31 & 0,92 & 1,60 & & 29 & 0,88 & $3,09^{* *}$ \\
\hline $\mathrm{L}$ vs $(\mathrm{K}+2 \mathrm{E})$ & 1 & 0,11 & 0,28 & 1 & 0,07 & 0,12 & & 1 & 1,25 & 4,40 * \\
\hline $\mathrm{K}$ vs $2 \mathrm{E}$ & 1 & 0,52 & 1,37 & 1 & 0,19 & 0,33 & & 1 & 1,01 & 3,55 \\
\hline E1 vs E2 & 1 & 7,56 & 19,85 ** & 1 & 7,56 & 13,14 & ** & 1 & 10,82 & $38,11 * *$ \\
\hline $\mathrm{L}$ & 28 & 0,34 & 0,90 & 28 & 0,74 & 1,28 & & 26 & 0,48 & 1,67 \\
\hline $\mathrm{GxO}$ & 31 & 0,38 & 1,84 * & 31 & 0,58 & 2,78 & ** & 29 & 0,28 & 1,66 \\
\hline Fehler & 98 & 0,21 & & 98 & 0,21 & & & 37 & 0,17 & \\
\hline
\end{tabular}

${ }^{* / * \star}$ signifikant bei $p=0,05 / 0,01,{ }^{a} \mathrm{G}$ : Genotypen, L: Linien, K: Kontrolle, E: Elter, O: Standorte

In der Befallsstärke der Linien gab es eine deutliche Variation. In der Kreuzung 1 wurden Boniturnoten zwischen 2,25 und 4,00 beobachtet, in der Kreuzung 2 zwischen 2,00 und 4,50 und in der Kreuzung 3 zwischen 1,37 und 3,00. Deutlich ist der Unterschied im Befall der jeweiligen beiden Eltern zu erkennen (Abb. 12). Sabina wies einen geringen Befall an zwei Standorten auf, gefolgt von CR1 im Mittel über zwei Standorte. Die beiden kanadischen Sorten Saffire und AC Sunset waren recht stark befallen. In den ersten beiden Kreuzungen wiesen nur wenige Linien einen geringeren Befall als die jeweilige Kontrolle auf (Kreuzung 1: zwei Linien, Kreuzung 2: fünf Linien). Viele Linien zeigten jedoch die gleiche Boniturnote wie die Kontrolle. In der ersten Kreuzung waren es zehn und in der zweiten Kreuzung sieben Linien. In der dritten Kreuzung waren zwölf Linien im Alternaria-Blattfleckenbefall besser als die Kontrolle. In den drei Kreuzungen waren keine Linien signifikant weniger befallen als die Kontrolle. In keiner Kreuzung wurde im Mittel über die Orte ein Selektionserfolg (L-K) beobachtet (Abb. 12 und Tab. 14). 

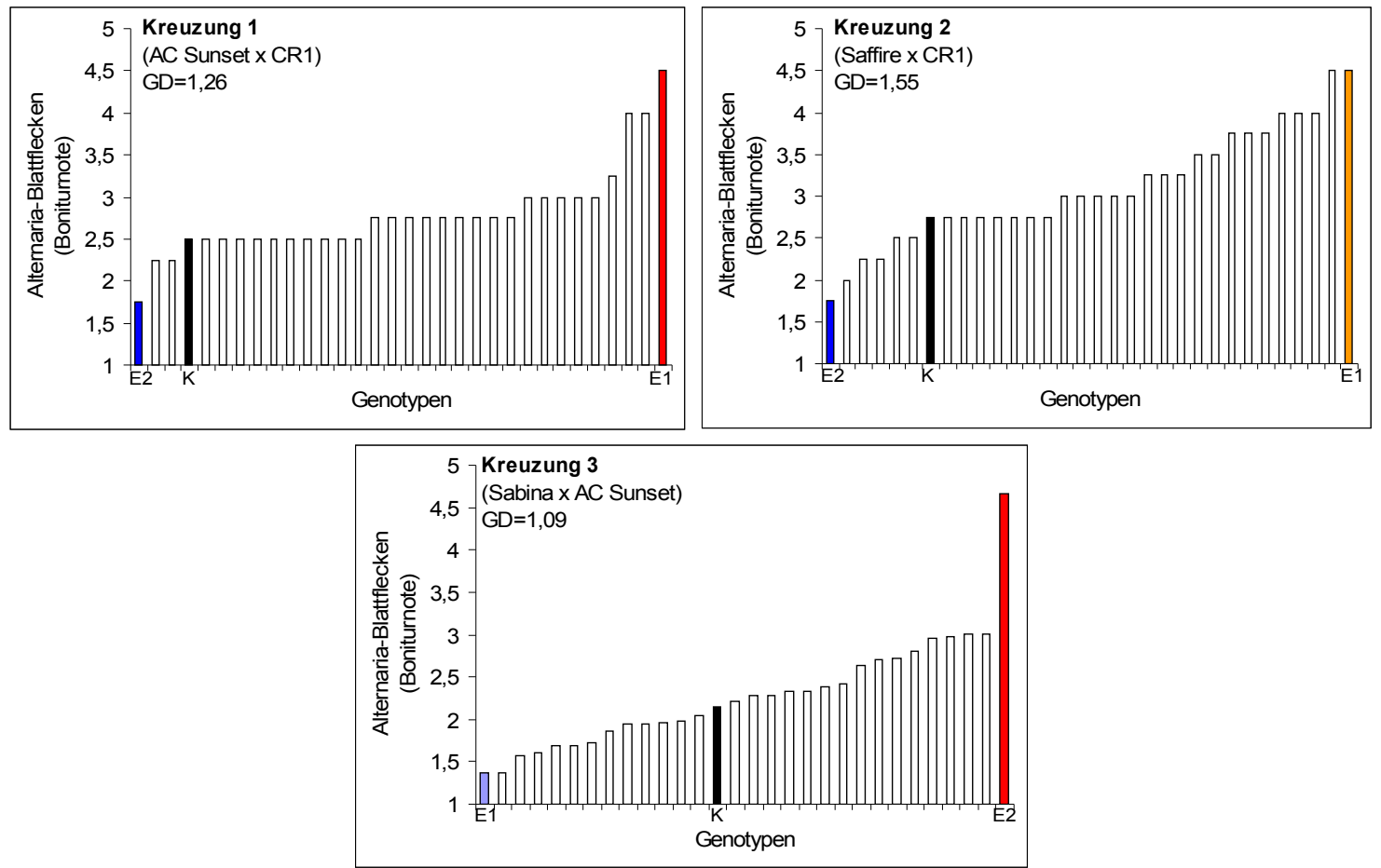

GD: Grenzdifferenz $(0,05)$

Abb. 12: Alternaria-Blattflecken, Boniturnoten der 29 (27) Linien, der Kontrolle (K) und der Eltern (E1, E2), gemittelt über zwei Versuchsstandorte

An den Daten der einzelnen Orte ist erkennbar, dass AC Sunset und Saffire an beiden Orten stark befallen waren. Meist konnte kein Effekt der Selektion beobachtet werden, nur in der dritten Kreuzung in Göttingen war die Selektion erfolgreich (Tab. 14). 
Tab. 14: Alternaria-Blattflecken (Note), Befall an den Versuchsstandorten und im Mittel der Orte

\begin{tabular}{lccc}
\hline & \multicolumn{2}{c}{ Standorte $^{\text {a }}$} & Mittel \\
\cline { 2 - 3 } & Gö & Ho & \\
\cline { 2 - 3 } Kreuzung 1 & & & 4,50 \\
Elter 1 (AC Sunset) & 5,00 & 4,00 & 1,75 \\
Elter 2 (CR1) & 1,50 & 2,00 & 2,78 \\
Mittel der Linien (L) & 2,69 & 2,86 & 2,50 \\
Kontrolle (K) & 2,50 & 2,50 & 0,28 \\
L - K & 0,19 & 0,36 & \\
Anzahl L besser als K & 5,00 & 5,00 & \\
Kreuzung 2 & & & 4,50 \\
Elter 1 (Saffire) & 5,00 & 4,00 & 1,75 \\
Elter 2 (CR1) & 1,50 & 2,00 & 3,11 \\
Mittel der Linien (L) & 3,40 & 2,83 & 2,75 \\
Kontrolle (K) & 3,00 & 2,50 & 0,36 \\
L - K & 0,40 & 0,33 & \\
Anzahl L besser als K & 7,00 & 4,00 & 1,37 \\
Kreuzung 3 & & & 4,66 \\
Elter 1 (Sabina) & 1,01 & 1,72 & 2,24 \\
Elter 2 (AC Sunset) & 3,97 & 5,34 & 2,14 \\
Mittel der Linien (L) & 1,92 & 2,56 & 0,10 \\
Kontrolle (K) & 2,12 & 2,16 & \\
L - K & $-0,20$ & 0,40 & \\
Anzahl L besser als K & 19,00 & 6,00 & \\
\hline
\end{tabular}

${ }^{a}$ Gö: Göttingen, Ho: Hohenheim, Wi: Wilmersdorf, Da: Darzau

\section{Fusarium}

Die Linien unterschieden sich in allen drei Kreuzungen hoch signifikant von dem Mittel aus der Kontrolle und den beiden Eltern. In den ersten beiden Kreuzungen war zudem die Kontrolle unterschiedlich zum Mittel der Eltern. AC Sunset und CR1, die Eltern der Kreuzung 1, waren ebenfalls hoch signifikant verschieden. Unterschiede zwischen den Linien zeigten die Kreuzungen 1 und 3. In der zweiten Kreuzung trat eine Interaktion zwischen den Genotypen, also den Linien plus Eltern und Kontrolle und den Standorten auf (siehe Tab. 15). 
Tab. 15: Ergebnisse der Varianzanalyse für das Merkmal Fusarium (\%) an vier (Kreuzung 3: zwei) Standorten 2006

\begin{tabular}{|c|c|c|c|c|c|c|c|c|c|c|}
\hline \multirow[b]{2}{*}{ Varianzursache $^{a}$} & \multicolumn{3}{|c|}{ Kreuzung 1} & \multicolumn{4}{|c|}{ Kreuzung 2} & \multicolumn{3}{|c|}{ Kreuzung 3} \\
\hline & FG & $M Q$ & F-Wert & FG & MQ & F-We & & FG & $M Q$ & F-Wert \\
\hline$\overline{\mathrm{G}}$ & 31 & 113,76 & $5,89^{* *}$ & 31 & 77,21 & 1,83 & * & 29 & 92,72 & 2,36 \\
\hline L vs ( & 1 & 497,52 & $25,78^{* *}$ & 1 & 360,54 & 8,55 & ** & 1 & 729,71 & 18,57 \\
\hline $\mathrm{Kv}$ & 1 & & 4,36 * & 1 & 222 & 5,29 & * & 1 & 5 & 0,08 \\
\hline E1 v & 1 & 492,35 & $25,51^{* *}$ & 1 & & 1,54 & & 1 & 5,83 & 0,15 \\
\hline L & 28 & 59 & 4,54 ** & 28 & 62 & 1,48 & & 26 & 75,01 & 1,91 * \\
\hline $\mathrm{G} \times \mathrm{C}$ & 93 & 19,30 & 0,70 & 93 & 42,16 & 1,54 & ** & 29 & 39,29 & 1,56 \\
\hline Fehler & 195 & 27,38 & & 195 & 27,38 & & & 35 & 25,16 & \\
\hline
\end{tabular}

${ }^{* / *}$ signifikant bei $p=0,05 / 0,01,{ }^{a}$ G: Genotypen, L: Linien, K: Kontrolle, E: Elter, O: Standorte

In keiner Kreuzung gab es Linien, die weniger Befall aufwiesen als die Kontrolle (Abb. 13). Bis auf AC Sunset in der Kreuzung 1 zeigten die Eltern in jeder Kreuzung stärkeren Befall als die jeweilige Kontrolle. Eine große Variation im Befall der Linien lag in allen drei Kreuzungen vor. In der ersten Kreuzung waren zwischen 7,83\% und 26,20\% Pflanzen in einer Parzelle befallen, in der zweiten Kreuzung zwischen $9,59 \%$ und $24,33 \%$ und in der dritten Kreuzung zwischen $6,95 \%$ und $29,61 \%$.
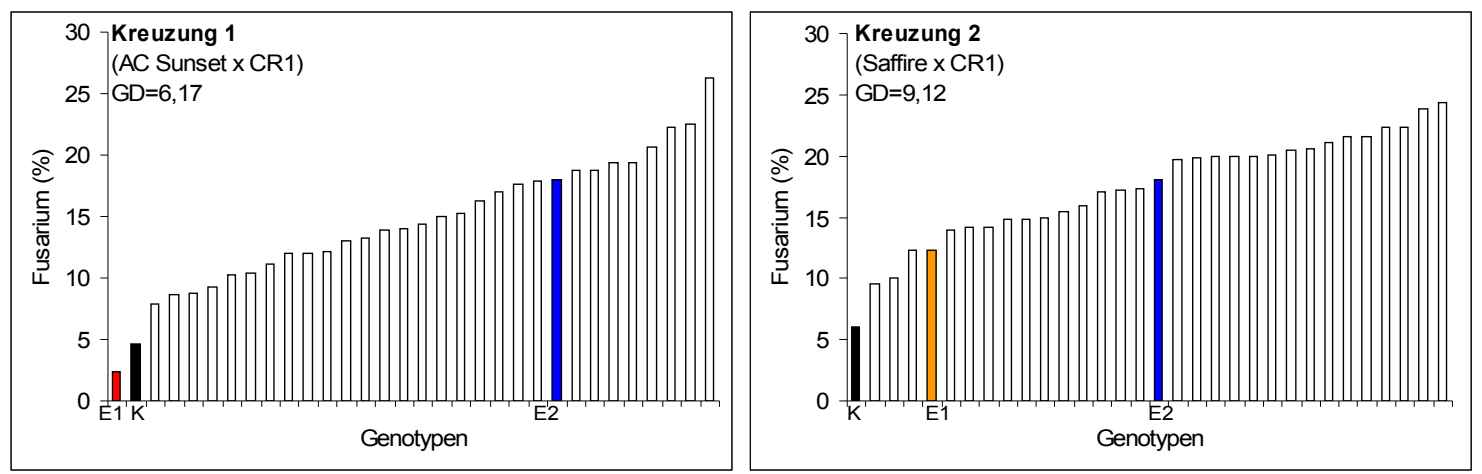

30 Kreuzung 3

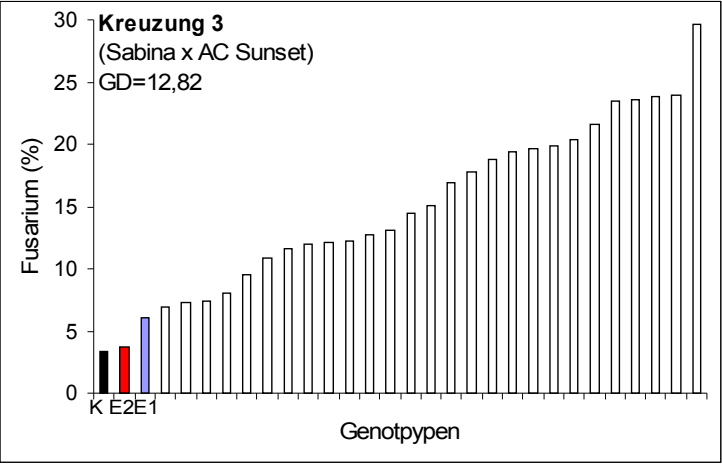

GD: Grenzdifferenz $(0,05)$

Abb. 13: Fusarium (\%) der 29 (27) Linien, der Kontrolle (K) und der Eltern (E1, E2), gemittelt über vier (zwei) Versuchsstandorte 
Der Befall mit Fusarium war in Darzau sehr gering. Sehr stark war er an den niederschlagsreicheren Standorten Hohenheim und Göttingen. An allen Standorten waren nur wenige Linien weniger befallen als die Kontrolle. In Göttingen war in allen drei Kreuzungen keine Linie besser als die Kontrolle (Tab. 16).

Tab. 16: Fusarium (\%), Befall an den Versuchsstandorten und im Mittel der Orte

\begin{tabular}{lccccc}
\hline & \multicolumn{3}{c}{ Standorte $^{\text {a }}$} & \\
\cline { 2 - 4 } & Gö & Ho & Wi & Da & Mittel \\
\hline Kreuzung 1 & & & & & \\
Elter 1 (AC Sunset) & 4,16 & 4,35 & 1,19 & 0,00 & 2,43 \\
Elter 2 (CR1) & 21,85 & 25,74 & 20,31 & 4,29 & 18,05 \\
Mittel der Linien (L) & 19,79 & 20,45 & 15,32 & 4,81 & 15,09 \\
Kontrolle (K) & 3,44 & 11,91 & 1,54 & 1,45 & 4,59 \\
L - K & 16,35 & 8,54 & 13,78 & 3,36 & 10,51 \\
Anzahl L besser als K & 0,00 & 3,00 & 0,00 & 5,00 & \\
Kreuzung 2 & & & & & \\
Elter 1 (Saffire) & 9,95 & 22,02 & 9,76 & 7,64 & 12,34 \\
Elter 2 (CR1) & 21,85 & 25,74 & 20,31 & 4,29 & 18,05 \\
Mittel der Linien (L) & 24,38 & 26,59 & 15,47 & 5,18 & 17,91 \\
Kontrolle (K) & 2,57 & 16,82 & 2,61 & 2,21 & 6,05 \\
L - K & 21,81 & 9,77 & 12,86 & 2,97 & 11,85 \\
Anzahl L besser als K & 0,00 & 4,00 & 1,00 & 5,00 & \\
Kreuzung 3 & & & & & \\
Elter 1 (Sabina) & 3,88 & 8,28 & & & 6,08 \\
Elter 2 (AC Sunset) & 0,19 & 7,14 & & & 3,67 \\
Mittel der Linien (L) & 16,70 & 15,29 & & & 15,99 \\
Kontrolle (K) & 1,81 & 4,91 & & & 12,36 \\
L - K & 14,89 & 10,38 & & & \\
Anzahl L besser als K & 0,00 & 2,00 & & & \\
\hline
\end{tabular}

${ }^{a}$ Gö: Göttingen, Ho: Hohenheim, Wi: Wilmersdorf, Da: Darzau

\section{Ölgehalt}

Die Daten für die Varianzanalyse des Ölgehaltes zeigen, dass sich die Eltern der zweiten Kreuzung (Saffire und CR1) hoch signifikant voneinander unterschieden. Die Linien der ersten und zweiten Kreuzung unterschieden sich ebenfalls hoch signifikant voneinander. In allen drei Kreuzungen gab es hoch signifikante Interaktionen zwischen den Genotypen und den Standorten (siehe Tab. 17). 
Tab. 17: Ergebnisse der Varianzanalyse für das Merkmal Ölgehalt (\%) an vier (Kreuzung 3: zwei) Standorten 2006

\begin{tabular}{|c|c|c|c|c|c|c|c|c|c|c|c|}
\hline \multirow{3}{*}{$\begin{array}{l}\text { Varianzursache }^{a} \\
\mathrm{G}\end{array}$} & \multicolumn{4}{|c|}{ Kreuzung 1} & \multicolumn{4}{|c|}{ Kreuzung 2} & \multicolumn{3}{|c|}{ Kreuzung 3} \\
\hline & FG & MQ & $F-W$ & & FG & MQ & $F-W e$ & & FG & $\mathbf{M Q}$ & F-Wert \\
\hline & 31 & 7,34 & 1,97 & ** & 31 & 6,61 & 2,18 & ** & 29 & 5,16 & 0,88 \\
\hline L vs $(K+2 E)$ & 1 & 0,89 & 0,24 & & 1 & 4,71 & 1,56 & & 1 & 0,16 & 0,03 \\
\hline $\mathrm{K}$ vs $2 \mathrm{E}$ & 1 & 2,14 & 0,57 & & 1 & 0,53 & 0,17 & & 1 & 5,55 & 0,95 \\
\hline E1 vs E2 & 1 & 14,20 & 3,82 & & 1 & 21,45 & 7,08 & $* *$ & 1 & 6,15 & 1,05 \\
\hline L & 28 & 7,51 & 2,02 & ** & 28 & 6,37 & 2,10 & ** & 26 & 5,30 & 0,90 \\
\hline $\mathrm{GxO}$ & 93 & 3,72 & 1,92 & ** & 93 & 3,03 & 1,56 & ** & 29 & 5,86 & $2,44^{* *}$ \\
\hline Fehler & 217 & 1,94 & & & 217 & 1,94 & & & 43 & 2,40 & \\
\hline
\end{tabular}

${ }^{* *}$ signifikant bei $p=0,01,{ }^{a}$ G: Genotypen, L: Linien, K: Kontrolle, E: Elter, O: Standorte

Abb. 14 verdeutlicht, dass die Eltern aller drei Kreuzungen unterschiedlichen Ölgehalt aufwiesen, wenn dies auch nur in der zweiten Kreuzung signifikant war. Auffällig ist, dass AC Sunset, gemessen am Durchschnitt der vier Orte, einen Ölgehalt von $25,1 \%$ besaß, in der dritten Kreuzung jedoch einen relativ niedrigen Ölgehalt von 18,5\% zeigte, was später in der Betrachtung der Daten an den einzelnen Orten nochmal erläutert wird. In allen drei Kreuzungen variierten die Ölgehalte der Linien relativ stark. In Kreuzung 1 lagen sie zwischen 20,2\% und 26,2\%, in der Kreuzung 2 zwischen 21,4\% und 26,9\% und in der Kreuzung 3 zwischen $17,0 \%$ und $23,6 \%$. In den Kreuzungen 1 und 2 wiesen jeweils 23 Linien einen höheren Ölgehalt als die Kontrolle auf. In der Kreuzung 3 waren es nur fünf Linien. Signifikant besser waren in der ersten und zweiten Kreuzung jeweils zwei Linien, in der dritten Kreuzung keine. In den Kreuzungen 1 und 2 stieg der Ölgehalt durch die Selektion, in der dritten Kreuzung war das Mittel der Linien im Ölgehalt geringer als die Kontrolle (Tab. 18). 

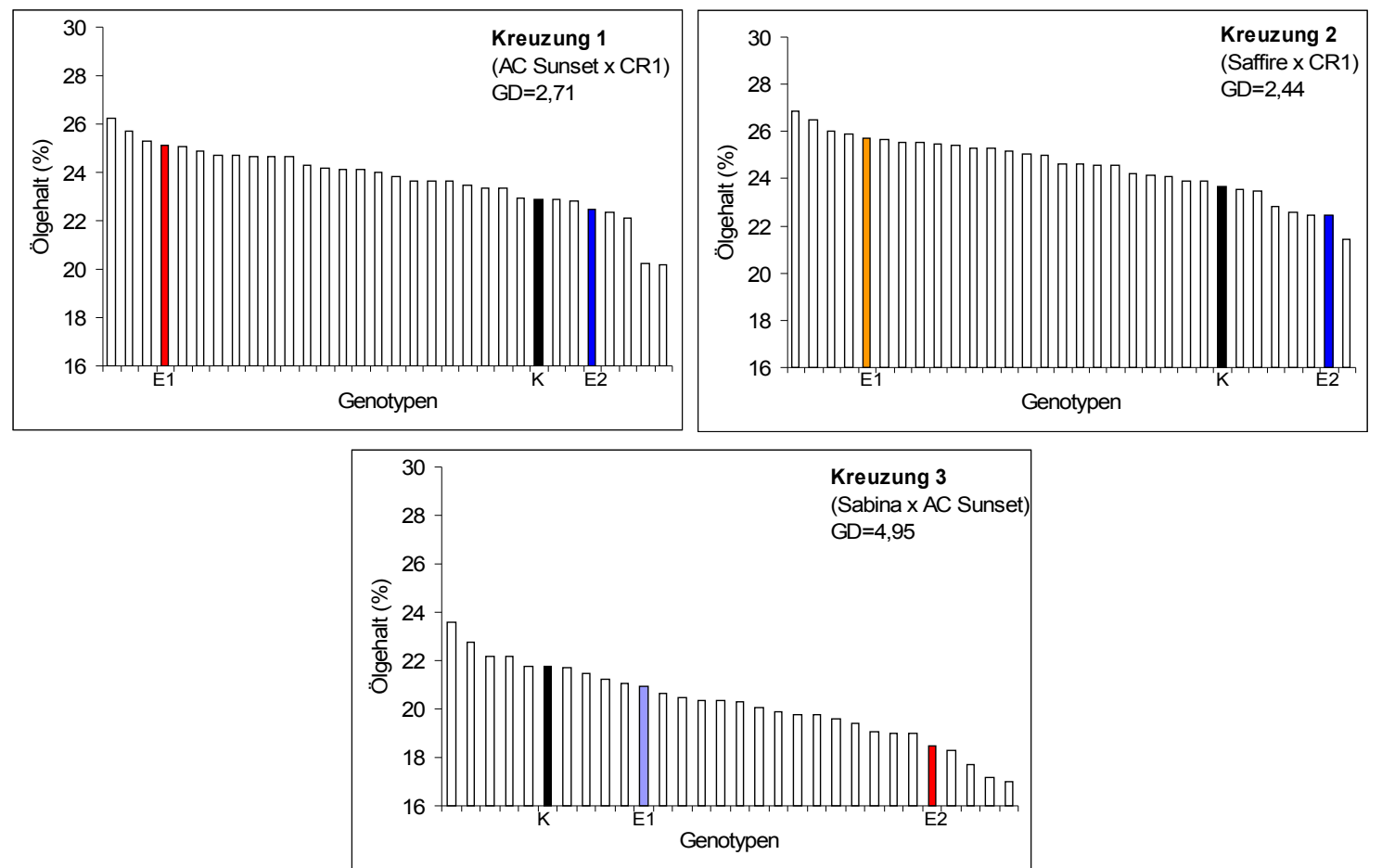

GD: Grenzdifferenz $(0,05)$

Abb. 14: Ölgehalt (\%) der 29 (27) Linien, der Kontrolle (K) und der Eltern (E1, E2), gemittelt über vier (zwei) Versuchsstandorte

Der Selektionserfolg war in den Kreuzungen und an den Standorten unterschiedlich. An den einzelnen Orten wiesen zahlreiche Linien in den Kreuzungen 1 und 2 einen höheren Ölgehalt als die Kontrolle auf. Wilmersdorf wich in der Kreuzung 1 und Göttingen in Kreuzung 2 etwas ab. AC Sunset (Elter 1 in Kreuzung 1 und Elter 2 in Kreuzung 3) zeigte in Göttingen im Vergleich zu den anderen Standorten einen sehr niedrigen Ölgehalt. Dies erklärt die oben erwähnten großen Unterschiede im Ölgehalt dieses Elters in Kreuzung 1 und 3 (Tab. 18). 
Tab. 18: Ölgehalt (\%), Daten der Versuchsstandorte und im Mittel der Orte

\begin{tabular}{|c|c|c|c|c|c|}
\hline & \multicolumn{4}{|c|}{ Standorte $^{a}$} & \multirow[b]{2}{*}{ Mittel } \\
\hline & Gö & Ho & $\mathbf{W i}$ & Da & \\
\hline \multicolumn{6}{|l|}{ Kreuzung 1} \\
\hline Elter 1 (AC Sunset) & 16,50 & 27,74 & 28,68 & 27,60 & 25,13 \\
\hline Elter 2 (CR1) & 19,02 & 21,70 & 30,95 & 18,19 & 22,47 \\
\hline Mittel der Linien (L) & 19,03 & 24,51 & 27,64 & 23,95 & 23,78 \\
\hline Kontrolle (K) & 18,46 & 23,24 & 28,21 & 21,70 & 22,90 \\
\hline$L-K$ & 0,57 & 1,27 & $-0,57$ & 2,25 & 0,88 \\
\hline Anzahl L besser als $\mathrm{K}$ & 19,00 & 18,00 & 10,00 & 27,00 & \\
\hline \multicolumn{6}{|l|}{ Kreuzung 2} \\
\hline Elter 1 (Saffire) & 18,92 & 27,19 & 32,16 & 24,69 & 25,74 \\
\hline Elter 2 (CR1) & 19,02 & 21,70 & 30,95 & 18,19 & 22,47 \\
\hline Mittel der Linien (L) & 20,63 & 25,98 & 27,59 & 24,25 & 24,61 \\
\hline Kontrolle (K) & 21,27 & 24,67 & 26,53 & 22,16 & 23,66 \\
\hline $\mathrm{L}-\mathrm{K}$ & $-0,64$ & 1,31 & 1,06 & 2,09 & 0,95 \\
\hline Anzahl L besser als $\mathrm{K}$ & 8,00 & 21,00 & 24,00 & 28,00 & \\
\hline \multicolumn{6}{|l|}{ Kreuzung 3} \\
\hline Elter 1 (Sabina) & 20,00 & 21,89 & & & 20,95 \\
\hline Elter 2 (AC Sunset) & 14,91 & 22,02 & & & 18,47 \\
\hline Mittel der Linien (L) & 18,96 & 21,48 & & & 20,22 \\
\hline Kontrolle (K) & 19,41 & 24,08 & & & 21,75 \\
\hline$L-K$ & $-0,45$ & $-2,60$ & & & $-1,53$ \\
\hline Anzahl L besser als $\mathrm{K}$ & 10,00 & 6,00 & & & \\
\hline
\end{tabular}

${ }^{a}$ Gö: Göttingen, Ho: Hohenheim, Wi: Wilmersdorf, Da: Darzau

\section{EP-Ertrag}

Die Varianzanalyse für die Daten des bereinigten Einzelpflanzenertrages (EPErtrag) zeigte, dass sich die Eltern der ersten Kreuzung signifikant voneinander unterschieden. Außerdem gab es hoch signifikante Interaktionen zwischen den Standorten und den Genotypen in den ersten beiden Kreuzungen (Tab. 19).

Tab. 19: Ergebnisse der Varianzanalyse für das Merkmal EP-Ertrag (g) an vier (Kreuzung 3: zwei) Standorten 2006

\begin{tabular}{|c|c|c|c|c|c|c|c|c|c|c|}
\hline \multirow[b]{2}{*}{ Varianzursache $^{\text {a }}$} & \multicolumn{4}{|c|}{ Kreuzung 1} & \multicolumn{3}{|c|}{ Kreuzung 2} & \multicolumn{3}{|c|}{ Kreuzung 3} \\
\hline & FG & $M Q$ & F-Wer & & FG & $M Q$ & F-Wert & FG & MQ & F-Wert \\
\hline $\bar{G}$ & 31 & 36,52 & 1,30 & & 31 & 37,62 & 1,14 & 29 & 72,60 & 1,80 \\
\hline$L$ vs $(K+2 E)$ & 1 & 35,68 & 1,27 & & 1 & 9,30 & 0,28 & 1 & 129,29 & 3,20 \\
\hline $\mathrm{K}$ vs $2 \mathrm{E}$ & 1 & 69,46 & 2,48 & & 1 & 0,13 & 0,00 & 1 & 33,77 & 0,84 \\
\hline E1 vs E2 & 1 & 187,31 & 6,68 & * & 1 & 26,43 & 0,80 & 1 & 29,21 & 0,72 \\
\hline $\mathrm{L}$ & 28 & 29,99 & 1,07 & & 28 & 40,37 & 1,22 & 26 & 73,58 & 1,82 \\
\hline $\mathrm{GxO}$ & 93 & 28,05 & 2,39 & ** & 93 & 32,98 & 2,81 ** & 29 & 40,37 & 1,63 \\
\hline Fehler & 203 & 11,72 & & & 203 & 11,72 & & 33 & 24,78 & \\
\hline
\end{tabular}

${ }^{*} /{ }^{* *}$ signifikant bei $p=0,05 / 0,01,{ }^{a} \mathrm{G}$ : Genotypen, L: Linien, K: Kontrolle, E: Elter, O: Standorte 
Die Variation in den Linien hinsichtlich des EP-Ertrages war besonders in Kreuzung 3 groß (zwischen 10,13 g und 36,74 g). Auch in den beiden anderen Kreuzungen variierten die Werte stark: Kreuzung 1: zwischen 13,93 g und 24,57 g, Kreuzung 2: zwischen 12,73 g und 25,33 g. In der ersten Kreuzung erzielten neun Linien einen höheren EP-Ertrag als die Kontrolle, in der zweiten Kreuzung 19 und in der dritten 17. Signifikant besser als die Kontrolle war in der dritten Kreuzung nur eine Linie. In den beiden anderen Kreuzungen wies keine Linie einen signifikant höheren EP-Ertrag auf. Indirekt hat sich die Selektion auf hohen Ölgehalt und geringen Krankheitsbefall unterschiedlich stark auf den EP-Ertrag der Kreuzungen ausgewirkt. In den Kreuzungen 2 und 3 lag der mittlere EP-Ertrag der Linien über dem der Kontrolle, in der ersten Kreuzung wurde kein Zuwachs beim EP-Ertrag beobachtet. Der EP-Ertrag von AC Sunset war im Vergleich zu den anderen Eltern relativ gering (Abb. 15 und Tab. 20).
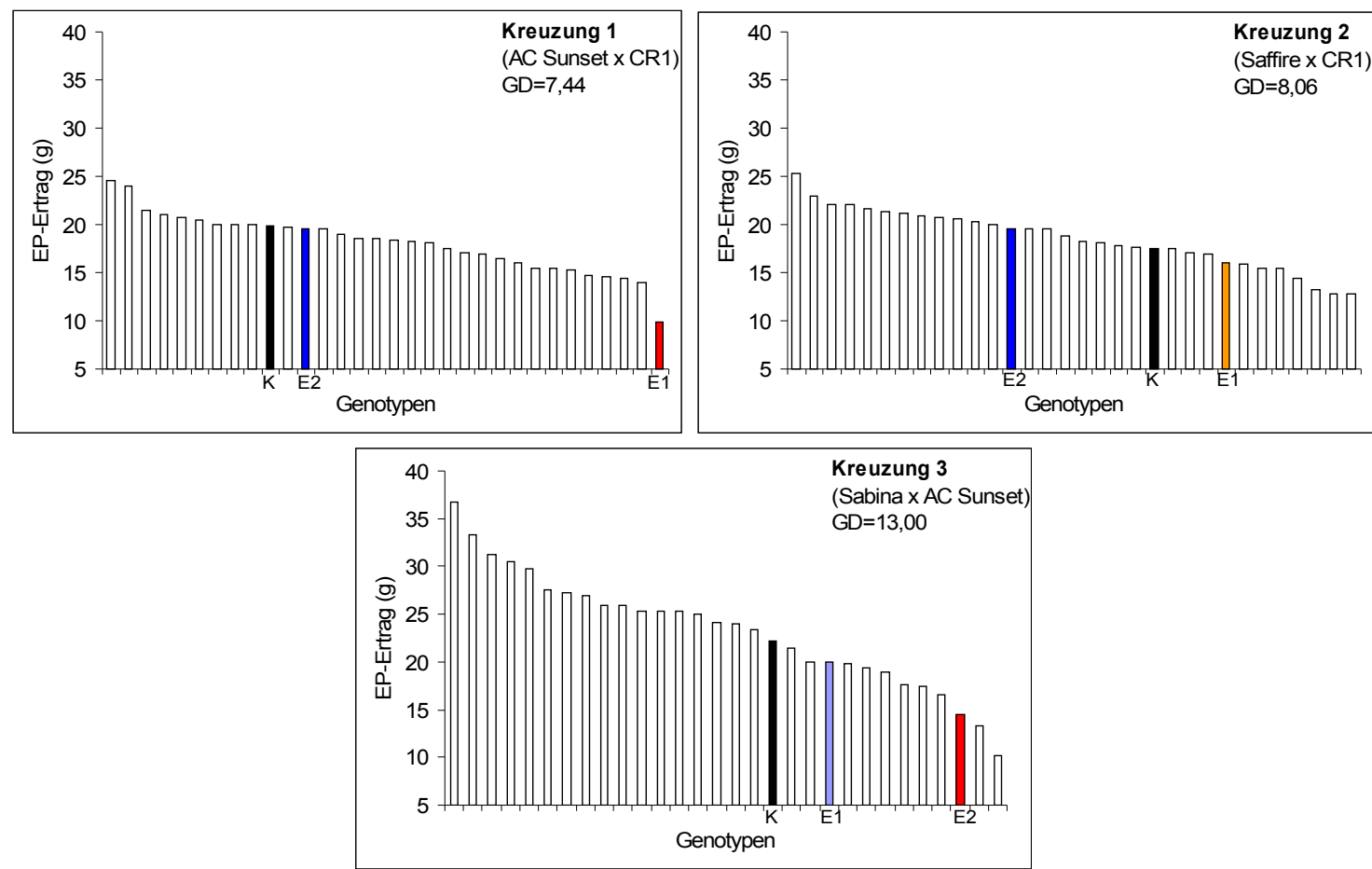

GD: Grenzdifferenz $(0,05)$

Abb. 15: EP-Ertrag (g) der 29 (27) Linien, der Kontrolle (K) und der Eltern (E1, E2), gemittelt über vier (zwei) Versuchsstandorte

Anhand der Daten der einzelnen Standorte wird deutlich, dass die EP-Erträge in Hohenheim höher waren, da hier pro Parzelle nur fünf gut entwickelte Einzelpflanzen geerntet wurden. Auffällig ist, dass in Darzau die EP-Erträge 
insgesamt geringer waren als an den anderen Standorten. Der EP-Ertrag von AC Sunset war in der ersten Kreuzung an drei der vier Standorte gering. Auch in der dritten Kreuzung wies AC Sunset in Göttingen einen relativ geringen EPErtrag auf (Tab. 20).

Tab. 20: EP-Ertrag (g), Daten der Versuchsstandorte und im Mittel der Orte

\begin{tabular}{lccccc}
\hline & \multicolumn{4}{c}{ Standorte $^{\text {a }}$} & \\
\cline { 2 - 4 } & Gö & Ho & Wi & Da & Mittel \\
\hline Kreuzung 1 & & & & & \\
Elter 1 (AC Sunset) & 7,20 & 19,42 & 6,86 & 6,21 & 9,92 \\
Elter 2 (CR1) & 16,86 & 39,14 & 16,10 & 6,30 & 19,60 \\
Mittel der Linien (L) & 16,97 & 36,35 & 12,76 & 7,02 & 18,27 \\
Kontrolle (K) & 19,53 & 40,00 & 12,40 & 7,53 & 19,87 \\
L - K & $-2,56$ & $-3,65$ & 0,36 & $-0,51$ & $-1,59$ \\
Anzahl L besser als K & 5,00 & 10,00 & 12,00 & 12,00 & \\
Kreuzung 2 & & & & & \\
Elter 1 (Saffire) & 15,34 & 25,87 & 12,86 & 9,79 & 15,97 \\
Elter 2 (CR1) & 16,86 & 39,14 & 16,10 & 6,30 & 19,60 \\
Mittel der Linien (L) & 19,40 & 34,70 & 12,84 & 7,59 & 18,63 \\
Kontrolle (K) & 20,33 & 30,65 & 12,09 & 7,17 & 17,56 \\
L - K & $-0,93$ & 4,05 & 0,75 & 0,42 & 1,07 \\
Anzahl L besser als K & 8,00 & 20,00 & 17,00 & 20,00 & \\
Kreuzung 3 & & & & & \\
Elter 1 (Sabina) & 18,71 & 21,10 & & & 19,91 \\
Elter 2 (AC Sunset) & 11,29 & 17,71 & & & 14,50 \\
Mittel der Linien (L) & 14,94 & 32,61 & & 23,77 \\
Kontrolle (K) & 14,41 & 30,06 & & & 22,24 \\
L - K & 0,53 & 2,55 & & & \\
Anzahl L besser als K & 13,00 & 16,00 & & & \\
\hline
\end{tabular}

${ }^{a}$ Gö: Göttingen, Ho: Hohenheim, Wi: Wilmersdorf, Da: Darzau

\section{TKG}

Die varianzanalytischen Daten für das TKG, dargestellt in Tab. 21, zeigen, dass sich in der Kreuzung 2 die Kontrolle vom Mittel der Eltern signifikant unterschied. In der ersten Kreuzung unterschieden sich die beiden Eltern signifikant voneinander und zwischen den Linien gab es in allen drei Kreuzungen signifikante Unterschiede. Genotyp-Umwelt-Interaktionen traten in der Kreuzung 1 hoch signifikant auf und in der Kreuzung 3 signifikant. 
Tab. 21: Ergebnisse der Varianzanalyse für das Merkmal TKG (g) an vier (Kreuzung 3: zwei) Standorten 2006

\begin{tabular}{|c|c|c|c|c|c|c|c|c|c|c|c|c|}
\hline \multirow{3}{*}{ Varianzursache $^{\text {a }}$} & \multicolumn{4}{|c|}{ Kreuzung 1} & \multicolumn{4}{|c|}{ Kreuzung 2} & \multicolumn{4}{|c|}{ Kreuzung 3} \\
\hline & FG & $M Q$ & $F-W e$ & & FG & MQ & F-We & & FG & MQ & F-We & \\
\hline & 31 & 13,51 & 4,68 & $* *$ & 31 & 13,32 & 6,67 & ** & 29 & 15,47 & 4,47 & ** \\
\hline$L$ vs $(K+2 E)$ & 1 & 9,67 & 3,35 & & 1 & 5,73 & 2,87 & & 1 & 5,07 & 1,46 & \\
\hline $\mathrm{K} v \mathrm{~s}$ & 1 & 0,17 & 0,06 & & 1 & 25,83 & 12,93 & $* *$ & 1 & 1,61 & 0,47 & \\
\hline E1 vs & 1 & 51,01 & 17,68 & $* *$ & $T$ & 4,96 & 2,48 & & 1 & 7,29 & 2,11 & \\
\hline $\mathrm{L}$ & 28 & 12,79 & 4,43 & $* *$ & 28 & 13,44 & 6,73 & $* *$ & 26 & 16,72 & 4,83 & ** \\
\hline $\mathrm{GxO}$ & 93 & 2,88 & 1,78 & $* *$ & 93 & 2,00 & 1,23 & & 29 & 3,46 & 2,03 & * \\
\hline Fehler & 194 & 1,63 & & & 194 & 1,63 & & & 43 & 1,70 & & \\
\hline
\end{tabular}

*/** signifikant bei $p=0,05 / 0,01,{ }^{a}$ G: Genotypen, L: Linien, K: Kontrolle, E: Elter, O: Standorte

Die Variation im TKG der Linien war in allen drei Kreuzungen sehr groß. In der ersten Kreuzung lag das TKG zwischen 28,1 g und 35,4 g, in der zweiten Kreuzung zwischen $28,8 \mathrm{~g}$ und $36,2 \mathrm{~g}$ und in der dritten Kreuzung zwischen $24,0 \mathrm{~g}$ und $37,0 \mathrm{~g}$. In der Kreuzung 2 erreichten 22 Linien ein höheres TKG als die Kontrolle. In der dritten Kreuzung waren es 16 und in der ersten Kreuzung waren es acht Linien. Ein signifikant höheres TKG wiesen in der Kreuzung 2 elf und in der Kreuzung 3 zwei Linien auf. In der Kreuzung 1 war keine Linie signifikant besser. Die Selektion auf höheren Ölgehalt und geringere Krankheitsanfälligkeit wirkte sich in den Kreuzungen 2 und 3 auf das TKG positiv aus, in der Kreuzung 1 war das TKG des Linienmittels geringer als das der Kontrolle (Abb. 16 und Tab. 22). 

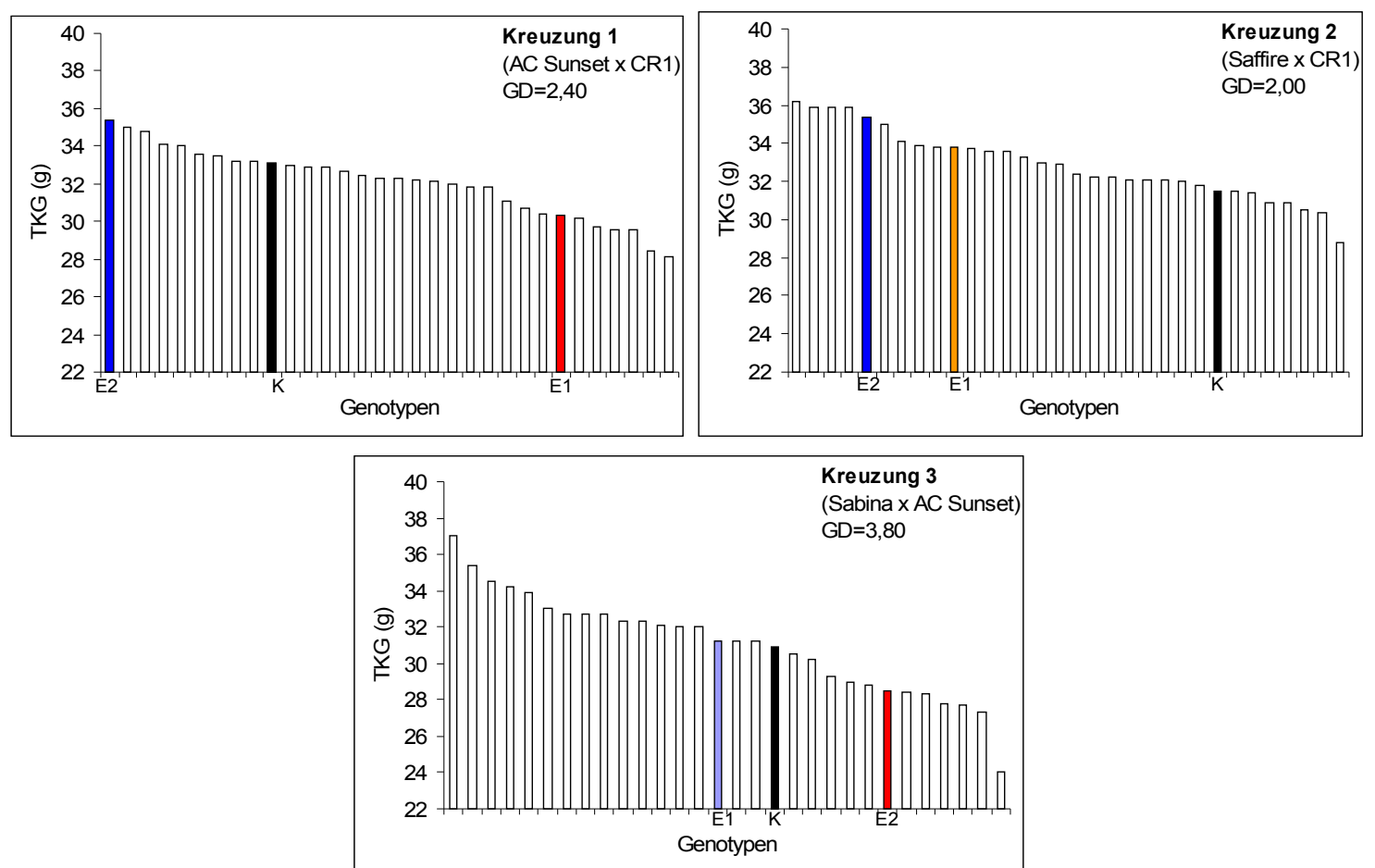

GD: Grenzdifferenz $(0,05)$

Abb. 16: TKG (g) der 29 (27) Linien, der Kontrolle (K) und der Eltern (E1, E2), gemittelt über vier (zwei) Versuchsstandorte

Die Daten der einzelnen Orte zeigen, dass das TKG in Hohenheim meist etwas höher war als an den anderen Orten (Tab. 22). Die indirekte Wirkung der Selektion auf das TKG war in den Kreuzungen unterschiedlich. In der Kreuzung 1 war an keinem Standort eine Steigerung beim TKG festzustellen. In den Kreuzungen 2 und 3 war das Linienmittel meist höher als das TKG der Kontrolle. Besonders in der Kreuzung 2 zeigten zahlreiche Linien ein höheres TKG als die Kontrolle. 
Tab. 22: TKG (g), Daten der Versuchsstandorte und im Mittel der Orte

\begin{tabular}{|c|c|c|c|c|c|}
\hline & \multicolumn{4}{|c|}{ Standorte $^{a}$} & \multirow[b]{2}{*}{ Mittel } \\
\hline & Gö & Ho & $\mathbf{W i}$ & $\mathrm{Da}$ & \\
\hline \multicolumn{6}{|l|}{ Kreuzung 1} \\
\hline Elter 1 (AC Sunset) & 21,50 & 35,00 & 33,20 & 31,60 & 30,33 \\
\hline Elter 2 (CR1) & 30,40 & 39,80 & 35,90 & 35,40 & 35,38 \\
\hline Mittel der Linien (L) & 26,84 & 36,11 & 34,62 & 30,39 & 31,99 \\
\hline Kontrolle $(\mathrm{K})$ & 27,40 & 38,40 & 36,10 & 30,50 & 33,10 \\
\hline$L-K$ & $-0,56$ & $-2,29$ & $-1,48$ & $-0,11$ & $-1,11$ \\
\hline Anzahl L besser als $\mathrm{K}$ & 11,00 & 5,00 & 8,00 & 15,00 & \\
\hline \multicolumn{6}{|l|}{ Kreuzung 2} \\
\hline Elter 1 (Saffire) & 26,30 & 37,60 & 37,60 & 33,70 & 33,80 \\
\hline Elter 2 (CR1) & 30,40 & 39,80 & 35,90 & 35,40 & 35,38 \\
\hline Mittel der Linien (L) & 28,19 & 36,28 & 34,82 & 32,00 & 32,82 \\
\hline Kontrolle (K) & 27,30 & 35,00 & 35,50 & 28,10 & 31,50 \\
\hline $\mathrm{L}-\mathrm{K}$ & 0,89 & 1,28 & $-0,68$ & 3,90 & 1,35 \\
\hline Anzahl L besser als $\mathrm{K}$ & 22,00 & 20,00 & 10,00 & 28,00 & \\
\hline \multicolumn{6}{|l|}{ Kreuzung 3} \\
\hline Elter 1 (Sabina) & 27,80 & 34,50 & & & 31,15 \\
\hline Elter 2 (AC Sunset) & 22,40 & 34,50 & & & 28,45 \\
\hline Mittel der Linien (L) & 28,32 & 33,95 & & & 31,14 \\
\hline Kontrolle (K) & 27,30 & 34,50 & & & 30,90 \\
\hline$L-K$ & 1,02 & $-0,55$ & & & 0,24 \\
\hline Anzahl L besser als $\mathrm{K}$ & 18,00 & 12,00 & & & \\
\hline
\end{tabular}

${ }^{a}$ Gö: Göttingen, Ho: Hohenheim, Wi: Wilmersdorf, Da: Darzau

\section{Schalenanteil}

Die Daten der Varianzanalyse für den Schalenanteil sind in der Tab. 23 dargestellt. Die Eltern der ersten beiden Kreuzungen waren signifikant unterschiedlich. Zudem unterschieden sich die Linien untereinander in der Kreuzung 1. Interaktionen zwischen den Genotypen und den Standorten gab es in der zweiten Kreuzung.

Tab. 23: Ergebnisse der Varianzanalyse für das Merkmal Schalenanteil (\%) an vier (Kreuzung 3: zwei) Standorten 2006

\begin{tabular}{|c|c|c|c|c|c|c|c|c|c|c|}
\hline \multirow[b]{2}{*}{ Varianzursache $^{a}$} & \multicolumn{3}{|c|}{ Kreuzung 1} & \multicolumn{4}{|c|}{ Kreuzung 2} & \multicolumn{3}{|c|}{ Kreuzung 3} \\
\hline & FG & MQ & F-Wert & FG & MQ & F-Wer & & FG & $M Q$ & F-Wert \\
\hline $\bar{G}$ & 31 & 41,64 & $2,73^{* *}$ & 31 & 24,60 & 1,39 & & 29 & 24,17 & 0,97 \\
\hline$L$ vs $(K+2 E)$ & 1 & 4,16 & 0,27 & 1 & 3,64 & 0,21 & & 1 & 31,97 & 1,28 \\
\hline $\mathrm{K}$ vs $2 \mathrm{E}$ & 1 & 3,44 & 0,22 & 1 & 24,73 & 1,39 & & 1 & 15,87 & 0,63 \\
\hline E1 vs E2 & 1 & 89,11 & $5,84 *$ & 1 & 81,41 & 4,58 & * & 1 & 0,48 & 0,02 \\
\hline L & 28 & 42,64 & $2,79 *$ & 28 & 23,31 & 1,31 & & 26 & 25,10 & 1,00 \\
\hline $\mathrm{GxO}$ & 93 & 15,27 & 1,23 & 93 & 17,76 & 1,43 & * & 29 & 24,99 & 1,60 \\
\hline Fehler & 192 & 12,42 & & 192 & 12,42 & & & 44 & 15,65 & \\
\hline
\end{tabular}

*** signifikant bei $p=0,05 / 0,01,{ }^{a} \mathrm{G}$ : Genotypen, L: Linien, K: Kontrolle, E: Elter, O: Standorte 
Die Wirkung der indirekten Selektion war in den Kreuzungen unterschiedlich. Die Anzahl der Linien, die einen geringeren Schalenanteil aufwiesen als die Kontrolle, war in Kreuzung 1 mit 16 Linien im Vergleich zu den anderen beiden Kreuzungen, in denen nur jeweils zwei Linien besser waren, recht hoch. Allerdings war keine der Linien signifikant besser. Der Schalenanteil der Linien wies eine Variation von $52,5 \%$ bis $64,4 \%$ (erste Kreuzung), $50,9 \%$ bis $60,4 \%$ (zweite Kreuzung) und $58,2 \%$ und $73,1 \%$ (dritte Kreuzung) auf (Abb. 17 und Tab. 24).
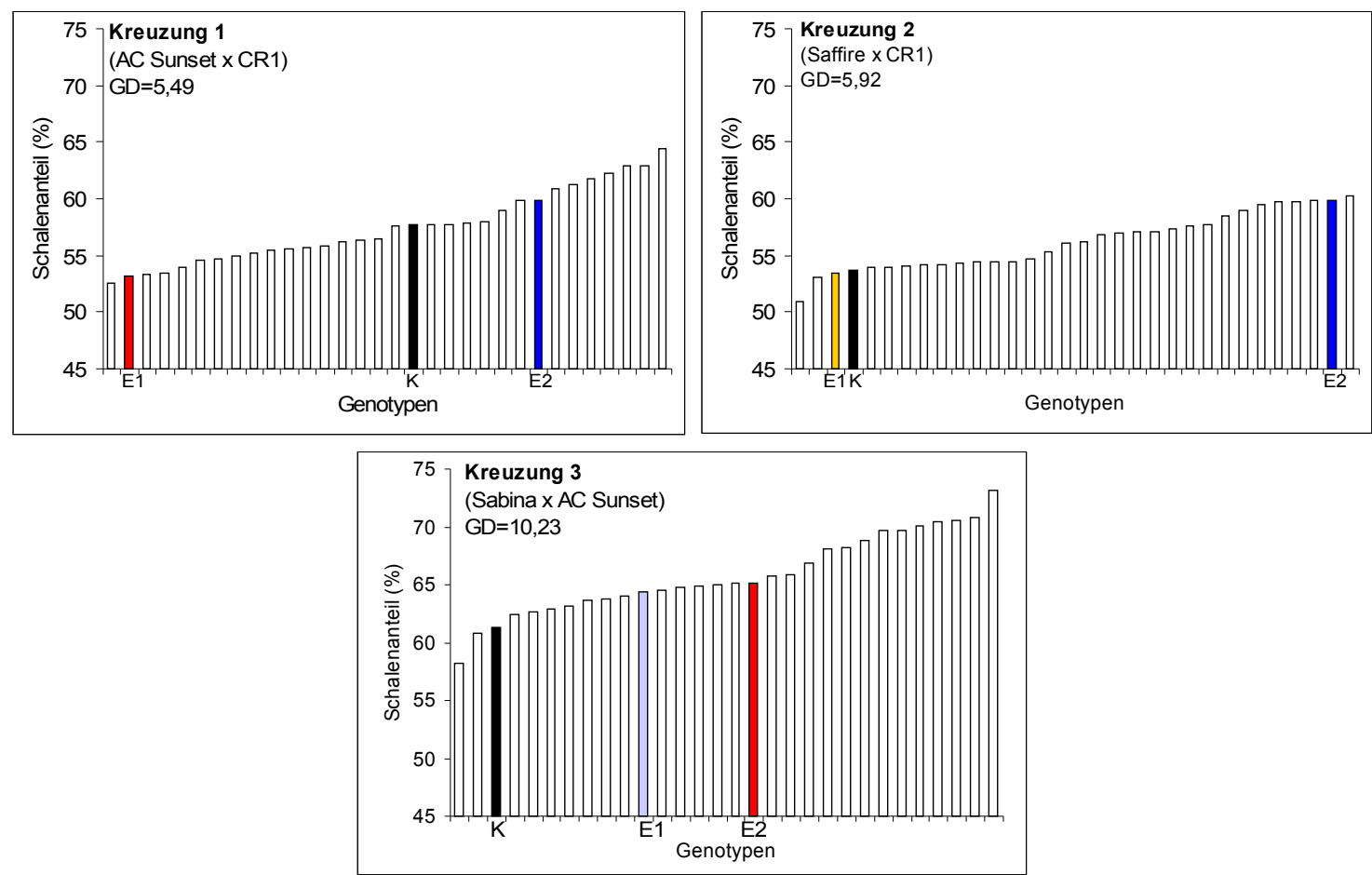

GD: Grenzdifferenz $(0,05)$

Abb. 17: Schalenanteil (\%) der 29 (27) Linien, der Kontrolle (K) und der Eltern (E1, E2), gemittelt über vier (zwei) Versuchsstandorte 
Die Anzahl der Linien, die einen geringeren Schalenanteil als die Kontrolle aufwiesen, war an den Orten sehr unterschiedlich. Das Mittel der Linien und auch die Werte der Eltern waren meist an den niederschlagsreicheren Standorten Göttingen und Hohenheim höher als in Wilmersdorf und Darzau (Tab. 24).

Tab. 24: Schalenanteil (\%), Daten der Versuchsstandorte und im Mittel der Orte

\begin{tabular}{|c|c|c|c|c|c|}
\hline & \multicolumn{4}{|c|}{ Standorte ${ }^{a}$} & \multirow[b]{2}{*}{ Mittel } \\
\hline & Gö & Ho & $\mathbf{W i}$ & $\mathrm{Da}$ & \\
\hline \multicolumn{6}{|l|}{ Kreuzung 1} \\
\hline Elter 1 (AC Sunset) & 68,75 & 44,07 & 49,79 & 50,20 & 53,20 \\
\hline Elter 2 (CR1) & 67,88 & 60,97 & 46,59 & 64,07 & 59,88 \\
\hline Mittel der Linien (L) & 71,84 & 54,76 & 51,02 & 52,53 & 57,54 \\
\hline Kontrolle (K) & 71,59 & 57,39 & 48,26 & 53,46 & 57,68 \\
\hline$L-K$ & 0,25 & $-2,63$ & 2,76 & $-0,93$ & $-0,14$ \\
\hline Anzahl $L$ besser als $K$ & 16,00 & 18,00 & 6,00 & 17,00 & \\
\hline \multicolumn{6}{|l|}{ Kreuzung 2} \\
\hline Elter 1 (Saffire) & 72,62 & 47,81 & 43,48 & 50,08 & 53,50 \\
\hline Elter 2 (CR1) & 67,88 & 60,97 & 46,59 & 64,07 & 59,88 \\
\hline Mittel der Linien (L) & 68,61 & 51,90 & 52,96 & 51,54 & 56,25 \\
\hline Kontrolle (K) & 67,45 & 46,29 & 47,57 & 53,26 & 53,64 \\
\hline$L-K$ & 1,16 & 5,61 & 5,39 & $-1,72$ & 2,61 \\
\hline Anzahl $\mathrm{L}$ besser als $\mathrm{K}$ & 13,00 & 3,00 & 0,00 & 20,00 & \\
\hline \multicolumn{6}{|l|}{ Kreuzung 3} \\
\hline Elter 1 (Sabina) & 66,18 & 62,70 & & & 64,44 \\
\hline Elter 2 (AC Sunset) & 74,30 & 55,96 & & & 65,13 \\
\hline Mittel der Linien (L) & 70,14 & 62,00 & & & 66,07 \\
\hline Kontrolle $(\mathrm{K})$ & 70,97 & 51,70 & & & 61,34 \\
\hline$L-K$ & $-0,83$ & 10,30 & & & 4,73 \\
\hline Anzahl L besser als $K$ & 18,00 & 1,00 & & & \\
\hline
\end{tabular}

${ }^{a}$ Gö: Göttingen, Ho: Hohenheim, Wi: Wilmersdorf, Da: Darzau

In der Abb. 18 ist zusammenfassend die Anzahl der Linien pro Kreuzung und Merkmal dargestellt, die besser als das Elternmittel, der bessere Elter oder die Kontrolle waren. Die Ergebnisse hingen sehr vom Merkmal und von der Kreuzung ab. 

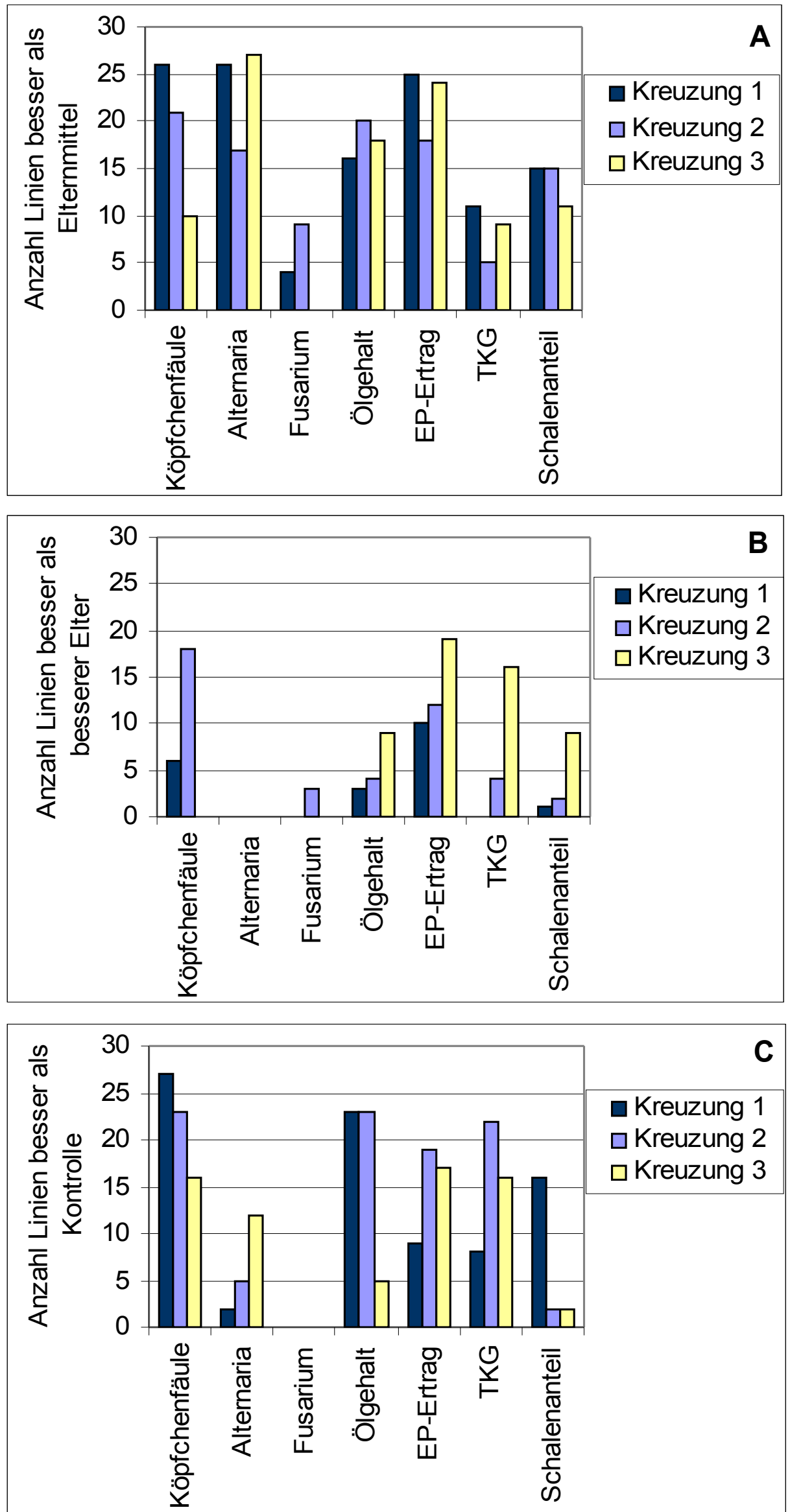

Abb. 18: Anzahl Linien pro Kreuzung und Merkmal besser als das Elternmittel (A), als der bessere Elter (B) oder als die Kontrolle (C) 


\section{Merkmalskorrelationen}

In den Tab. 25 bis 27 finden sich die Korrelationen der einzelnen Merkmale in den drei Kreuzungen. Der Ölgehalt war in allen drei Kreuzungen signifikant mit dem Schalenanteil negativ korreliert. Korrelationen zwischen anderen Merkmalen waren in den Kreuzungen unterschiedlich. In der zweiten und dritten Kreuzung zeichnete sich eine Beziehung zwischen Ölgehalt und EPErtrag ab, die in dem einem Fall negativ und in dem anderen positiv war. Je höher der Ölgehalt in der zweiten Kreuzung war, desto niedriger war das TKG. In der ersten Kreuzung korrelierte der Befall mit Köpfchenfäule positiv mit dem TKG. In der zweiten Kreuzung zeichnete sich eine negative Beziehung zwischen dem Alternaria-Blattfleckenbefall und dem EP-Ertrag ab.

Tab. 25: Korrelationen der Merkmale in der Kreuzung 1

\begin{tabular}{lcccccc}
\hline & Köpfchenfäule & Alternaria & Fusarium & Ölgehalt & EP-Ertrag & TKG \\
\hline Alternaria & 0,188 & & & & & \\
Fusarium & 0,158 & 0,041 & & & & \\
Ölgehalt & $-0,088$ & 0,217 & 0,051 & & & \\
EP-Ertrag & $-0,046$ & $-0,334$ & $-0,171$ & 0,074 & & \\
TKG & $0,521^{* *}$ & 0,023 & $0,370^{*}$ & $-0,227$ & 0,060 & \\
Schalenanteil & $-0,234$ & $-0,149$ & $-0,125$ & $-0,667^{* *}$ & $-0,232$ & $-0,160$ \\
\hline
\end{tabular}

Tab. 26: Korrelationen der Merkmale in der Kreuzung 2

\begin{tabular}{lcccccc}
\hline & Köpfchenfäule & Alternaria & Fusarium & Ölgehalt & EP-Ertrag & TKG \\
\hline Alternaria & $-0,082$ & & & & & \\
Fusarium & 0,045 & 0,035 & & & & \\
Ölgehalt & $-0,076$ & 0,339 & 0,021 & & & \\
EP-Ertrag & 0,071 & $-0,377^{*}$ & 0,014 & $-0,372^{*}$ & & \\
TKG & 0,322 & 0,336 & 0,980 & $-0,553^{* *}$ & $0,388^{*}$ & \\
Schalenanteil & 0,034 & $-0,125$ & $-0,086$ & $-0,505^{* *}$ & 0,329 & 0,280 \\
\hline
\end{tabular}

Tab. 27: Korrelationen der Merkmale in der Kreuzung 3

\begin{tabular}{lcccccc}
\hline & Köpfchenfäule & Alternaria & Fusarium & Ölgehalt & EP-Ertrag & TKG \\
\hline Alternaria & $-0,036$ & & & & & \\
Fusarium & 0,167 & $-0,139$ & & & & \\
Ölgehalt & $-0,156$ & 0,024 & 0,284 & & & \\
EP-Ertrag & $-0,315$ & 0,089 & 0,299 & $0,581^{* *}$ & & \\
TKG & 0,037 & 0,162 & 0,336 & 0,210 & $0,649^{* *}$ & \\
Schalenanteil & 0,017 & $-0,116$ & $-0,006$ & $-0,691^{* *}$ & $-0,268$ & 0,089 \\
\hline
\end{tabular}




\subsubsection{Methode der natürlichen Selektion}

In 2006 wurden die neun Ramschherkünfte, die der natürlichen Selektion unterlagen, an vier Standorten zusammen mit den drei unselektierten Kontrollen verglichen. Für einen gleichmäßigen Feldaufgang wurde vor der Aussaat die Keimfähigkeit des Erntegutes aus 2005 untersucht. Es waren deutliche Unterschiede in den Kreuzungen zu beobachten. Bei den Kreuzungen 1 und 3 der Ramschherkünfte aus Hohenheim handelt es sich um die Keimfähigkeit des Saatgutes aus 2004 (Tab. 28). Obwohl anhand des TKG und der Keimfähigkeit die Aussaatstärke für jede Parzelle einzeln errechnet wurde, war der Feldaufgang an den Standorten und in den Ramschherkünften unterschiedlich (Tab. 29).

Tab. 28: Keimfähigkeit (\%) des Erntegutes aus 2005 (bei R Ho Kreuzung 1 und Kreuzung 3 aus 2004)

\begin{tabular}{lccc}
\hline Kreuzung & R Gö & R Ho & R Wi \\
\hline Kreuzung 1 & 26 & 48 & 52 \\
Kreuzung 2 & 82 & 42 & 84 \\
Kreuzung 3 & 28 & 39 & 36 \\
\hline
\end{tabular}

Herkunftsorte: R Gö: Göttingen, R Ho: Hohenheim, R Wi: Wilmersdorf

Tab. 29: Feldaufgang (Pflanzen pro $\mathrm{m}^{2}$ ) an den vier Versuchsstandorten

\begin{tabular}{lccccc}
\hline Standort & R Gö & R Ho & R Wi & Kontrolle & Mittel \\
\hline Göttingen & 19,9 & 29,9 & 20,0 & 25,7 & 23,9 \\
Hohenheim & 9,4 & 17,7 & 10,1 & 21,0 & 14,6 \\
Wilmersdorf & 10,8 & 22,2 & 13,6 & 19,5 & 16,5 \\
Darzau & 7,4 & 10,1 & 8,8 & 11,8 & 9,5 \\
\hline
\end{tabular}

Herkunftsorte: R Gö: Göttingen, R Ho: Hohenheim, R Wi: Wilmersdorf

In Tab. 30 sind die Ergebnisse der natürlichen Selektion an den drei Herkunftsorten (Göttingen, Hohenheim und Wilmersdorf) sowie die Daten der Kontrollen dargestellt, gemittelt über die Standorte und Kreuzungen. Außerdem wurde eine Varianzanalyse durchgeführt (siehe als Beispiel: Ölgehalt dargestellt im Anhang Tab. A XVII). Die Varianzkomponenten der Herkunftsorte $(H)$, der Interaktion zwischen Herkünften und Kreuzungen $(H \times C)$ und der Interaktion zwischen den Herkünften und den Standorten $(\mathrm{HxO})$ sind für alle Merkmale in Tab. 30 dargestellt. 
Die natürliche Selektion führte zu einem späteren Blühbeginn, Blühende und Reifezeitpunkt. Hier waren hoch signifikante Unterschiede zwischen den Ramschherkünften und der Kontrolle zu verzeichnen. Die Pflanzen blühten im Durchschnitt länger, was aber nicht durch Signifikanz zu belegen war.

Die natürliche Selektion führte zu kürzeren Pflanzen. Die Unterschiede waren jedoch nicht signifikant. Statt dessen trat eine signifikante Interaktion zwischen den Herkünften und den Kreuzungen auf.

Der Befall mit Köpfchenfäule nahm im Vergleich zur Kontrolle bei allen drei Ramschherkünften signifikant ab.

Die natürliche Selektion führte in Göttingen und Wilmersdorf zu höherem Befall mit Fusarium, sodass eine Betrachtung der über die Standorte aufgegliederten Daten später folgt.

Der Ölgehalt veränderte sich im Laufe der natürlichen Selektion nicht signifikant.

Hoch signifikante Unterschiede zeigten sich beim Merkmal EP-Ertrag. Dieser war im Mittel der einzelnen Ramschherkünfte höher als der Mittelwert der Kontrolle. Das gleiche Ergebnis war für das TKG zu beobachten, bei dem die natürliche Selektion zu einem Anstieg führte. Der Schalenanteil nahm zu, was aber nicht signifikant belegt werden konnte. Die Merkmale Pflanzenlänge, Befall mit Köpfchenfäule und Fusarium sowie der Ölgehalt, der EP-Ertrag und das TKG werden im Folgenden genauer erläutert. Der Befall mit Alternaria trat nur an zwei Standorten differenziert auf und wird aufgrund der fehlenden Signifikanz nicht eingehender beschrieben. 
Tab. 30: Ergebnisse der natürlichen Selektion der drei Herkunftsorte und der Kontrolle, im Mittel über die Standorte und Kreuzungen sowie die Varianzkomponenten

\begin{tabular}{|c|c|c|c|c|c|c|c|c|c|c|c|}
\hline \multirow[b]{2}{*}{ Merkmal a $^{a}$} & \multicolumn{4}{|c|}{ Herkunftsort ${ }^{\text {b }}$} & \multirow{2}{*}{$\begin{array}{c}\text { GD } \\
(0,05)^{c}\end{array}$} & \multicolumn{6}{|c|}{ Varianzkomponenten ${ }^{d}$} \\
\hline & R Gö & R Ho & $\mathbf{R} \mathbf{W i}$ & Kontrolle & & H & & $\mathrm{HxC}$ & & $\mathrm{HxO}$ & \\
\hline Blühbeginn & 199,83 & 199,42 & 200,08 & 198,71 & 36 & 0,35 & $* *$ & 0,14 & $* *$ & 0,22 & ** \\
\hline lühende & 218,88 & 218,46 & 219,29 & 217,00 & 9 & 0,85 & $* *$ & 0,02 & & 0,74 & * \\
\hline ühc & 9,04 & 19,04 & 19,21 & 9 & 04 & 0,04 & & $-0,22$ & & 0,28 & \\
\hline eifezei & 240,08 & 240,08 & 240,71 & 239,21 & 0,44 & 0,36 & $* *$ & 0,16 & . & 0,44 & ** \\
\hline Pflanzenlänge & 86,42 & 86,83 & 86,54 &, 04 & 62 & $-0,28$ & & 4,64 & ** & $-1,97$ & \\
\hline Köpfc & 2,67 & 2,63 & 1,96 & 08 & 0,33 & 0,20 & $* *$ & 0,00 & & 0,01 & \\
\hline Alternaria & 2,42 & 2,17 & 2,17 & 2,42 & 0,29 & 0,01 & & 0,00 & & 0,00 & \\
\hline usa & 7,52 & 1,78 & 3,13 & 2,16 & 1,85 & 6,57 & $* *$ & 15,79 & ** & 3,21 & * \\
\hline Ölgehalt & 22,39 & 23,35 & 22,32 & 23,15 & 1,07 & 0,13 & & 1,45 & $* *$ & 0,86 & * \\
\hline P-Ertrag & 15,96 & 13,99 & 16,04 & 13,02 & 1,87 & 1,80 & $* *$ & 1,77 & * & 1,25 & \\
\hline$K G$ & 34,28 & 33,79 & 35,29 & 33,1 & 0,89 & 0,76 & $* *$ & 0,07 & & 0,04 & \\
\hline Schalenanteil & 60,08 & 59,51 & 61,15 & 58,71 & 2,79 & 0,10 & & 0,42 & & $-0,80$ & \\
\hline
\end{tabular}

${ }^{a}$ Einheiten: Blühbeginn-Reifezeitpunkt: numerischer Tag, Pflanzenlänge: $\mathrm{cm}$, Köpfchenfäule und Alternaria: Note, Fusarium: \%, Ölgehalt: \%, EP-Ertrag und TKG: g, Schalenanteil: \% ${ }^{\mathrm{b}}$ Gö: Göttingen, Ho: Hohenheim, Wi: Wilmersdorf, ${ }^{\circ} \mathrm{GD}(0,05)$ : Grenzdifferenz, ${ }^{\mathrm{d}} \mathrm{H}$ : Herkunftsort, $\mathrm{HxC}$ : Interaktion zwischen Herkunftsort $(\mathrm{H})$ und Kreuzung $(\mathrm{C})$, $\mathrm{HxO}$ : Interaktion zwischen Herkunftsort $(\mathrm{H})$ und Standort $(\mathrm{O})$ */** signifikant bei $p=0,05 / 0,01$ (F-Test der mittleren Abweichungsquadrate)

\section{Pflanzenlänge}

Die Kontrolle der Kreuzung 3 wies die höchste Pflanzenlänge auf, gefolgt von den Kontrollen der Kreuzung 2 und 1. Interessant ist bei der Betrachtung der einzelnen Kreuzungen, dass die natürliche Selektion in der Kreuzung $3 \mathrm{zu}$ kürzeren Pflanzen und in der Kreuzung 2 zu längeren Pflanzen geführt hat (Tab. 31).

Insgesamt waren die Pflanzen 2006 an den niederschlagsreicheren Standorten Göttingen und Hohenheim länger (Tab. 32).

Tab. 31: Pflanzenlänge $(\mathrm{cm})$ der Ramschparzellen 2006 aufgegliedert in Kreuzungen

\begin{tabular}{lccccc}
\hline Kreuzung & R Gö & R Ho & R Wi & Mittel & Kontrolle \\
\hline Kreuzung 1 & 85,75 & 85,00 & 86,13 & 85,63 & 85,88 \\
Kreuzung 2 & 89,63 & 90,75 & 87,63 & 89,34 & 86,63 \\
Kreuzung 3 & 83,88 & 84,75 & 85,88 & 84,84 & 91,63 \\
Mittel & $\mathbf{8 6 , 4 2}$ & $\mathbf{8 6 , 8 3}$ & $\mathbf{8 6 , 5 4}$ & 86,60 & $\mathbf{8 8 , 0 4}$ \\
\hline
\end{tabular}

Herkunftsorte: R Gö: Göttingen, R Ho: Hohenheim, R Wi: Wilmersdorf 
Tab. 32: Pflanzenlänge $(\mathrm{cm})$ der Ramschparzellen 2006 aufgegliedert in Standorte

\begin{tabular}{lccccc}
\hline Standort & R Gö & R Ho & R Wi & Mittel & Kontrolle \\
\hline Göttingen & 101,67 & 104,17 & 100,83 & 102,22 & 103,33 \\
Hohenheim & 95,67 & 96,5 & 97,00 & 96,39 & 98,83 \\
Wilmersdorf & 75,83 & 75,83 & 76,67 & 76,11 & 75,83 \\
Darzau & 72,5 & 70,83 & 71,67 & 71,67 & 74,17 \\
Mittel & 86,42 & 86,83 & 86,54 & 86,60 & 88,04 \\
\hline
\end{tabular}

Herkunftsorte: R Gö: Göttingen, R Ho: Hohenheim, R Wi: Wilmersdorf

\section{Köpfchenfäule}

Die natürliche Selektion war für das Merkmal Köpfchenfäule erfolgreich. In allen Kreuzungen zeigten die Ramschparzellen der einzelnen Herkunftsorte einen geringeren Befall als die unselektierte Kontrolle (Tab. 33).

Die Boniturnoten von $\mathrm{R}$ Wi über die Standorte waren meist niedriger als die von R Ho und R Gö. Die natürliche Selektion war hier folglich sehr erfolgreich. Es könnte eine mögliche Anpassung an den Standort der Auslese gegeben haben, da in Wilmersdorf die Parzellen R Wi im Gegensatz zu R Gö und R Ho keinen Befall zeigten. Der Befall war allgemein am Standort Wilmersdorf relativ gering (vergleiche Tab. 34).

Tab. 33: Köpfchenfäule (Note) der Ramschparzellen 2006 aufgegliedert in Kreuzungen

\begin{tabular}{lccccc}
\hline Kreuzung & R Gö & R Ho & R Wi & Mittel & Kontrolle \\
\hline Kreuzung 1 & 2,88 & 2,88 & 1,88 & 2,55 & 3,25 \\
Kreuzung 2 & 2,50 & 2,63 & 2,25 & 2,46 & 3,13 \\
Kreuzung 3 & 2,63 & 2,38 & 1,75 & 2,25 & 2,88 \\
Mittel & $\mathbf{2 , 6 7}$ & $\mathbf{2 , 6 3}$ & $\mathbf{1 , 9 6}$ & 2,42 & $\mathbf{3 , 0 8}$ \\
\hline
\end{tabular}

Herkunftsorte: R Gö: Göttingen, R Ho: Hohenheim, R Wi: Wilmersdorf

Tab. 34: Köpfchenfäule (Note) der Ramschparzellen 2006 aufgegliedert in Standorte

\begin{tabular}{lccccc}
\hline Standort & $\mathbf{R}$ Gö & $\mathbf{R}$ Ho & $\mathbf{R}$ Wi & Mittel & Kontrolle \\
\hline Göttingen & $\mathbf{3 , 0 0}$ & 3,33 & 2,33 & 2,89 & 3,67 \\
Hohenheim & 2,50 & $\mathbf{2 , 1 7}$ & 2,17 & 2,28 & 3,00 \\
Wilmersdorf & 2,17 & 1,67 & $\mathbf{1 , 0 0}$ & 1,61 & 2,33 \\
Darzau & 3,00 & 3,33 & 2,33 & 2,89 & 3,33 \\
Mittel & 2,67 & 2,63 & 1,96 & 2,42 & 3,08 \\
\hline
\end{tabular}

Herkunftsorte: R Gö: Göttingen, R Ho: Hohenheim, R Wi: Wilmersdorf 


\section{Fusarium}

Die natürliche Selektion führte an den Herkunftsorten Göttingen und Wilmersdorf zu einem erhöhten Befall mit Fusarium. Die Daten der einzelnen Kreuzungen ergaben jedoch ein sehr uneinheitliches Bild. R1 Gö und R3 Gö zeigten einen sehr hohen Befall, wohingegen R2 Gö sehr gering befallen war. Genau gegenteilig verhielt es sich bei R Wi. Hier waren die Kreuzungen 1 und 3 gering befallen und die Kreuzung 2 sehr stark (Tab. 35).

Anhand der Daten über die Standorte, dargestellt in Tab. 36, ließ sich erkennen, dass der Befall an den niederschlagsreicheren Standorten Hohenheim und Göttingen im Mittel hoch war und in Darzau kaum Befall auftrat. In Hohenheim deutet der niedrige Befall der R Ho Parzellen auf eine mögliche Anpassung an den Standort der natürlichen Selektion hin, bei den anderen Ramschherkünften war dies jedoch nicht zu beobachten.

Tab. 35: Fusarium (\%) der Ramschparzellen 2006 aufgegliedert in Kreuzungen

\begin{tabular}{lccccc}
\hline Kreuzung & R Gö & R Ho & R Wi & Mittel & Kontrolle \\
\hline Kreuzung 1 & 9,76 & 2,11 & 0,93 & 4,27 & 1,28 \\
Kreuzung 2 & 1,07 & 0,78 & 8,16 & 3,34 & 1,26 \\
Kreuzung 3 & 11,72 & 2,45 & 0,31 & 4,83 & 3,94 \\
Mittel & $\mathbf{7 , 5 2}$ & $\mathbf{1 , 7 8}$ & $\mathbf{3 , 1 3}$ & 4,14 & $\mathbf{2 , 1 6}$ \\
\hline
\end{tabular}

Herkunftsorte: R Gö: Göttingen, R Ho: Hohenheim, R Wi: Wilmersdorf

Tab. 36: Fusarium (\%) der Ramschparzellen 2006 aufgegliedert in Standorte

\begin{tabular}{lccccc}
\hline Standort & $\mathbf{R}$ Gö & R Ho & $\mathbf{R ~ W i}$ & Mittel & Kontrolle \\
\hline Göttingen & $\mathbf{1 0 , 2 7}$ & 2,87 & 6,13 & 6,42 & 2,68 \\
Hohenheim & 13,06 & $\mathbf{2 , 9 5}$ & 4,33 & 6,78 & 2,85 \\
Wilmersdorf & 6,76 & 1,30 & $\mathbf{2 , 0 8}$ & 3,38 & 2,62 \\
Darzau & 0,00 & 0,00 & 0,00 & 0,00 & 0,50 \\
Mittel & 7,52 & 1,78 & 3,13 & 4,14 & 2,16 \\
\hline
\end{tabular}

Herkunftsorte: R Gö: Göttingen, R Ho: Hohenheim, R Wi: Wilmersdorf

\section{Ölgehalt}

In Göttingen und Wilmersdorf als Herkunftsorte führte die natürliche Selektion im Mittel zu einem leicht niedrigeren Ölgehalt, in Hohenheim dagegen zu einem etwas höheren. Die Unterschiede zur Kontrolle waren jedoch nur gering und nicht signifikant (Tab. 37).

Am Standort Göttingen erreichten die Prüfglieder den geringsten Ölgehalt, in Wilmersdorf im Mittel den höchsten. Auch hier waren Unterschiede zwischen den Standorten erkennbar. In Göttingen wiesen alle Ramschherkünfte einen 
höheren Ölgehalt als die Kontrolle auf, in Hohenheim alle einen niedrigeren. An den Standorten Wilmersdorf und Darzau hing es von der jeweiligen Herkunft des Ramsches ab (Tab. 38).

Tab. 37: Ölgehalt (\%) der Ramschparzellen 2006 aufgegliedert in Kreuzungen

\begin{tabular}{lllllc}
\hline Kreuzung & R Gö & R Ho & R Wi & Mittel & Kontrolle \\
\hline Kreuzung 1 & 24,86 & 22,81 & 23,16 & 23,61 & 23,45 \\
Kreuzung 2 & 23,12 & 25,19 & 22,02 & 23,44 & 23,86 \\
Kreuzung 3 & 19,19 & 22,05 & 21,78 & 21,01 & 22,13 \\
Mittel & $\mathbf{2 2 , 3 9}$ & $\mathbf{2 3 , 3 5}$ & $\mathbf{2 2 , 3 2}$ & 22,69 & $\mathbf{2 3 , 1 5}$ \\
\hline
\end{tabular}

Herkunftsorte: R Gö: Göttingen, R Ho: Hohenheim, R Wi: Wilmersdorf

Tab. 38: Ölgehalt (\%) der Ramschparzellen 2006 aufgegliedert in Standorte

\begin{tabular}{lccccc}
\hline Standort & $\mathbf{R}$ Gö & $\mathbf{R}$ Ho & $\mathbf{R}$ Wi & Mittel & Kontrolle \\
\hline Göttingen & $\mathbf{1 8 , 2 9}$ & 17,31 & 18,26 & 17,95 & 16,63 \\
Hohenheim & 22,81 & $\mathbf{2 4 , 8}$ & 22,82 & 23,48 & 26,36 \\
Wilmersdorf & 26,16 & 26,32 & $\mathbf{2 4 , 7 5}$ & 25,74 & 26,14 \\
Darzau & 22,30 & 24,96 & 23,46 & 23,57 & 23,47 \\
Mittel & 22,39 & 23,35 & 22,32 & 22,69 & 23,15 \\
\hline
\end{tabular}

Herkunftsorte: R Gö: Göttingen, R Ho: Hohenheim, R Wi: Wilmersdorf

\section{EP-Ertrag}

Die natürliche Selektion führte in fast allen Fällen zu einem erhöhten EP-Ertrag. Ein etwas geringerer EP-Ertrag war in den Parzellen des R1 Ho und des R3 Ho festzustellen. Das Saatgut dieser Prüfglieder stammte jedoch aus dem Jahr 2004 und unterlag nur einem Jahr natürlicher Selektion (Tab. 39).

Im Mittel über die Kreuzungen erreichten die R Gö und R Wi ähnliche Werte. Der EP-Ertrag war 2006 am Standort Göttingen am höchsten und in Darzau relativ niedrig. Eine Anpassung an den Standort der natürlichen Auslese wurde nicht festgestellt (Tab. 40).

Tab. 39: EP-Ertrag (g) der Ramschparzellen 2006 aufgegliedert in Kreuzungen

\begin{tabular}{lccccc}
\hline Kreuzung & R Gö & R Ho & R Wi & Mittel & Kontrolle \\
\hline Kreuzung 1 & 14,69 & 13,28 & 14,45 & 14,14 & 13,60 \\
Kreuzung 2 & 15,32 & 15,30 & 19,23 & 16,62 & 12,84 \\
Kreuzung 3 & 17,88 & 13,40 & 14,44 & 15,24 & 12,64 \\
Mittel & $\mathbf{1 5 , 9 6}$ & $\mathbf{1 3 , 9 9}$ & $\mathbf{1 6 , 0 4}$ & 15,33 & $\mathbf{1 3 , 0 2}$ \\
\hline
\end{tabular}

Herkunftsorte: R Gö: Göttingen, R Ho: Hohenheim, R Wi: Wilmersdorf 
Tab. 40: EP-Ertrag (g) der Ramschparzellen 2006 aufgegliedert in Standorte

\begin{tabular}{lccccc}
\hline Standort & $\mathbf{R}$ Gö & $\mathbf{R}$ Ho & $\mathbf{R ~ W i}$ & Mittel & Kontrolle \\
\hline Göttingen & $\mathbf{1 7 , 8 7}$ & 18,78 & 20,95 & 19,20 & 17,29 \\
Hohenheim & 19,18 & $\mathbf{1 3 , 5 8}$ & 18,47 & 17,08 & 11,95 \\
Wilmersdorf & 15,80 & 13,19 & $\mathbf{1 4 , 2 6}$ & 14,42 & 12,19 \\
Darzau & 11,01 & 10,43 & 10,47 & 10,64 & 10,68 \\
Mittel & 15,96 & 13,99 & 16,04 & 15,33 & 13,02 \\
\hline
\end{tabular}

Herkunftsorte: R Gö: Göttingen, R Ho: Hohenheim, R Wi: Wilmersdorf

\section{TKG}

Das TKG nahm in allen Kreuzungen an allen Herkunftsorten im Vergleich zur jeweiligen Kontrolle zu (Tab. 41). In Hohenheim wurden die höchsten Werte beobachtet. Gleichzeitig war der geringste Anstieg bei den R Ho an fast allen Standorten zu beobachten, Darzau bildete hier eine Ausnahme (Tab. 42)

Tab. 41: TKG (g) der Ramschparzellen 2006 aufgegliedert in Kreuzungen

\begin{tabular}{llllcc}
\hline Kreuzung & R Gö & R Ho & R Wi & Mittel & Kontrolle \\
\hline Kreuzung 1 & 33,53 & 33,23 & 35,63 & 34,13 & 33,09 \\
Kreuzung 2 & 34,61 & 33,75 & 34,42 & 34,26 & 33,38 \\
Kreuzung 3 & 34,70 & 34,38 & 35,83 & 34,97 & 32,83 \\
Mittel & 34,28 & 33,79 & 35,29 & 34,45 & 33,10 \\
\hline
\end{tabular}

Herkunftsorte: R Gö: Göttingen, R Ho: Hohenheim, R Wi: Wilmersdorf

Tab. 42: TKG (g) der Ramschparzellen 2006 aufgegliedert in Standorte

\begin{tabular}{lllllc}
\hline Standort & R Gö & R Ho & R Wi & Mittel & Kontrolle \\
\hline Göttingen & $\mathbf{2 7 , 6 7}$ & 26,72 & 28,96 & 27,78 & 26,17 \\
Hohenheim & 37,80 & $\mathbf{3 6 , 7 8}$ & 37,88 & 37,49 & 37,53 \\
Wilmersdorf & 37,14 & 36,60 & $\mathbf{3 8 , 1 8}$ & 37,31 & 35,98 \\
Darzau & 34,51 & 35,05 & 36,15 & 35,24 & 32,69 \\
Mittel & 34,28 & 33,79 & 35,29 & 34,45 & 33,10 \\
\hline
\end{tabular}

Herkunftsorte: R Gö: Göttingen, R Ho: Hohenheim, R Wi: Wilmersdorf 


\subsubsection{Einkornramschmethode}

Für den Einkornramsch wurden pro Kreuzung 50 F2-Pflanzen im Gewächshaus bis zur F6 einzelpflanzenweise weitergeführt. Die F7-Pflanzen wurden in 2006 in Doppelreihen zusammen mit den Eltern an zwei Standorten angebaut.

In Göttingen sind im Mittel in der ersten Kreuzung 66,6\%, in der zweiten 75,7\% und in der dritten 70,7\% der Pflanzen aufgelaufen. Am Standort Hohenheim war aus ungeklärten Gründen der Aufgang sehr schlecht (Kreuzung 1: 15,7\%, Kreuzung 2: 15,8\%, Kreuzung 3: 10,7\%), sodass der Versuch dort nicht weitergeführt und ausgewertet werden konnte.

In Tab. 43 sind die Daten der wichtigsten Merkmale für jede Kreuzung dargestellt. Der Elter 1 steht für die Mutter der Kreuzung (Kreuzung 1: AC Sunset, Kreuzung 2: Saffire, Kreuzung 3: Sabina) und der Elter 2 für den Vater (Kreuzung 1 und 2: CR1, Kreuzung 3: AC Sunset). Neben den Werten der Linien und Eltern sind die Anzahl der Linien, die besser waren als das Mittel der Eltern, und die Anzahl der Linien, die besser waren als der bessere Elter, aufgeführt. Insgesamt wurden 50 Linien getestet.

Beim Merkmal Köpfchenfäule waren deutliche Unterschiede zwischen den Kreuzungen festzustellen. Besonders in der ersten Kreuzung waren viele Linien weniger mit Köpfchenfäule befallen als das Elternmittel. Auch in der dritten Kreuzung war fast die Hälfte aller Linien besser. Nur eine Linie war in der zweiten Kreuzung weniger befallen. In dieser Kreuzung gab es keine Variation im Befall der Eltern. In allen drei Kreuzungen wiesen nur wenige Linien einen geringeren Befall auf als der bessere Elter.

Zahlreiche Linien zeigten geringeren Befall mit Alternaria-Blattflecken als das Elternmittel. In der Kreuzung 2 waren sogar 17 Linien besser als der bessere Elter.

Die Eltern zeigten kaum Fusariumbefall, eine Ausnahme bildete AC Sunset in der ersten Kreuzung. So waren in der zweiten und dritten Kreuzung nur wenige Linien geringer befallen als das Elternmittel und der bessere Elter. In der ersten Kreuzung waren fast alle Linien besser als das Elternmittel.

Die Werte für den Ölgehalt der Linien variierten stark. Viele Linien wiesen einen höheren Ölgehalt als das Elternmittel und als der bessere Elter auf. Die Kreuzung 3 zeigte im Linienmittel einen geringen Ölgehalt und beinhaltete auch nur wenige Linien, die über dem besseren Elter lagen. 
Die Variation im EP-Ertrag der Linien war sehr groß. Zahlreiche Linien erreichten einen höheren EP-Ertrag als das Elternmittel, in der zweiten Kreuzung auch als der bessere Elter. AC Sunset war in der Kreuzung 1 stark von Krankheiten befallen, sodass hier nur ein sehr geringer EP-Ertrag festgestellt wurde. Durch diesen Wert war das Elternmittel niedrig und fast alle Linien lagen über diesem EP-Ertrag.

Die Anzahl der Linien, die ein höheres TKG als das Elternmittel aufwiesen, war in den Kreuzungen unterschiedlich. In der ersten Kreuzung waren es weniger, in der zweiten und dritten über die Hälfte aller Linien. In der Kreuzung 3 erreichten fast die Hälfte aller Linien ein höheres TKG als der bessere Elter.

Der Schalenanteil der Linien zeigte eine große Spannweite. Im Mittel wiesen die Linien und die Eltern der Kreuzung 3 den höchsten Schalenanteil auf. Hier lagen mehrere Linien unter dem Mittelwert der Eltern und dem Schalenanteil des besseren Elters.

Die Variation in den Linien beim Ölgehalt, TKG und EP-Ertrag war groß. Die Verteilung der Linien in den einzelnen Klassen dieser Merkmale ist in Abb. 19 dargestellt. 
Tab. 43: Merkmalsdaten der 50 Linien und zwei Eltern pro Kreuzung

\begin{tabular}{|c|c|c|c|c|c|c|c|c|c|}
\hline \multirow{3}{*}{ Merkmal } & \multicolumn{4}{|c|}{ Linien } & \multicolumn{3}{|c|}{ Eltern } & \multicolumn{2}{|c|}{$\begin{array}{c}\text { Anzahl Linien } \\
\text { besser als }\end{array}$} \\
\hline & Min. & Max. & Mittel & SD ${ }^{a}$ & Elter 1 & Elter 2 & Mittel & Mittel & bessere \\
\hline & & & & & & & & Eltern & Elter \\
\hline \multicolumn{10}{|c|}{ Köpfchenfäule (Note) } \\
\hline Kreuzung 1 & 1,97 & 4,69 & 3,25 & 0,56 & 4,97 & 2,79 & 3,88 & 41,00 & 2,00 \\
\hline Kreuzung 2 & 2,00 & 5,00 & 3,32 & 0,54 & 3,00 & 3,00 & 3,00 & 1,00 & 1,00 \\
\hline Kreuzung 3 & 1,92 & 4,95 & 3,14 & 0,72 & 1,95 & 4,06 & 3,01 & 20,00 & 2,00 \\
\hline \multicolumn{10}{|c|}{ Alternaria-Blattflecken (Note) } \\
\hline Kreuzung 1 & 2,00 & 5,00 & 2,90 & 1,01 & 5,00 & 2,00 & 3,50 & 43,00 & 0,00 \\
\hline Kreuzung 2 & 1,53 & 5,23 & 2,96 & 0,97 & 5,15 & 2,69 & 3,92 & 43,00 & 17,00 \\
\hline Kreuzung 3 & 0,96 & 5,10 & 2,46 & 1,23 & 0,83 & 4,97 & 2,90 & 35,00 & 0,00 \\
\hline \multicolumn{10}{|c|}{ Fusarium (\%) } \\
\hline Kreuzung 1 & 0,00 & 15,38 & 1,32 & 4,84 & 25,00 & 0,00 & 12,50 & 47,00 & 0,00 \\
\hline Kreuzung 2 & 0,00 & 6,67 & 0,38 & 1,48 & 0,00 & 0,00 & 0,00 & 0,00 & 0,00 \\
\hline Kreuzung 3 & 0,00 & 6,78 & 0,43 & 1,76 & 0,28 & 0,28 & 0,28 & 6,00 & 6,00 \\
\hline \multicolumn{10}{|l|}{ Ölgehalt (\%) } \\
\hline Kreuzung 1 & 11,41 & 25,36 & 18,81 & 3,10 & 18,94 & 19,38 & 19,16 & 20,00 & 19,00 \\
\hline Kreuzung 2 & 9,99 & 25,60 & 19,72 & 3,31 & 20,57 & 20,26 & 20,42 & 22,00 & 22,00 \\
\hline Kreuzung 3 & 9,13 & 24,78 & 16,27 & 3,68 & 20,43 & 14,70 & 17,57 & 18,00 & 7,00 \\
\hline \multicolumn{10}{|l|}{ EP-Ertrag (g) } \\
\hline Kreuzung 1 & 9,87 & 40,62 & 28,50 & 7,41 & 0,29 & 33,53 & 16,91 & 49,00 & 10,00 \\
\hline Kreuzung 2 & 15,02 & 57,38 & 29,22 & 7,19 & 28,23 & 28,91 & 28,57 & 25,00 & 24,00 \\
\hline Kreuzung 3 & 7,93 & 46,72 & 24,94 & 8,14 & 31,02 & 14,14 & 22,58 & 29,00 & 8,00 \\
\hline \multicolumn{10}{|l|}{ TKG (g) } \\
\hline Kreuzung 1 & 22,60 & 32,90 & 28,11 & 3,11 & 25,00 & 34,30 & 29,65 & 15,00 & 0,00 \\
\hline Kreuzung 2 & 20,60 & 33,80 & 28,59 & 2,69 & 26,40 & 30,30 & 28,35 & 28,00 & 12,00 \\
\hline Kreuzung 3 & 20,00 & 34,60 & 26,84 & 3,15 & 27,60 & 24,20 & 25,90 & 31,00 & 23,00 \\
\hline \multicolumn{10}{|c|}{ Schalenanteil (\%) } \\
\hline Kreuzung 1 & 61,28 & 86,08 & 71,10 & 6,28 & 66,85 & 60,85 & 63,85 & 8,00 & 0,00 \\
\hline Kreuzung 2 & 54,56 & 80,51 & 66,99 & 6,56 & 65,70 & 59,31 & 62,51 & 13,00 & 7,00 \\
\hline Kreuzung 3 & 59,91 & 88,28 & 74,57 & 7,22 & 74,37 & 70,19 & 72,28 & 19,00 & 16,00 \\
\hline
\end{tabular}

a SD: Standardabweichung 

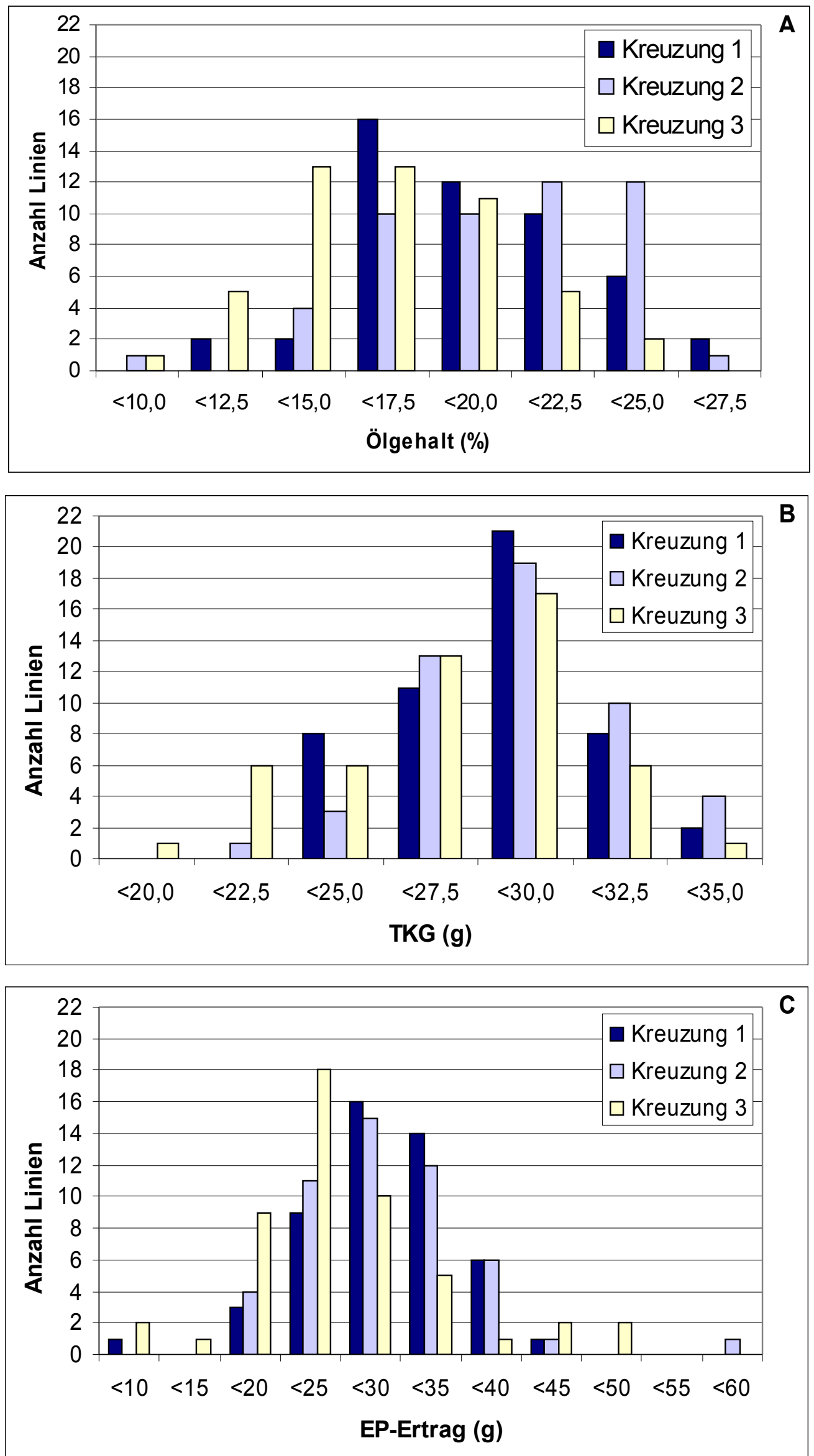

Abb. 19: Verteilung der Linien in den Ölgehaltsklassen (A), TKG-Klassen (B) und EP-Ertragsklassen (C) 


\subsection{Diskussion}

\subsubsection{Bewertung der Stammbaummethode}

Die Stammbaummethode wird bei Saflor nach Knowles (1989) besonders für hoch heritable Merkmale wie beispielsweise Krankheitsresistenz angewendet.

Pilzinfektionen wie Köpfchenfäule und Alternaria-Blattflecken sind weit verbreitet und können ernsthafte Verluste hervorrufen (Mündel et al. 2004). Daher stand ein niedriger Krankheitsbefall zusammen mit einem hohen Ölgehalt bei der Selektion im Vordergrund. Der Ertrag zählt sicherlich auch zu den agronomisch wichtigen Merkmalen. Bei einer in Deutschland kaum züchterisch bearbeiteten Pflanze wie Saflor sollten jedoch zunächst Linien mit verbessertem Ölgehalt und geringerem Krankheitsbefall entwickelt werden.

Es wurde jeweils ein kanadischer Elter (AC Sunset und Saffire), der einen hohen Ölgehalt aufwies, mit einem Elter, der geringeren Krankheitsbefall zeigte (Sabina und CR1), gekreuzt. AC Sunset und Saffire waren stärker mit Köpfchenfäule und Alternaria-Blattflecken befallen, wie es bereits in der Literatur erwähnt wurde (Mündel et al. 2004, Mündel 1996, Frick und Hebeisen 2005). Bei Sabina wurde, wie bei Frick und Hebeisen (2005) beschrieben, ein geringerer Befall beobachtet.

AC Sunset und Saffire erzielten an den niederschlagsärmeren Standorten (Wilmersdorf und Darzau) relativ hohe Ölgehalte. In Göttingen war der Ölgehalt bei AC Sunset jedoch sehr gering. Mündel et al. (2004) berichteten, dass der Befall mit Köpfchenfäule eine Minderung im Ölgehalt bewirkt. Die Merkmale Köpfchenfäule und Ölgehalt waren nicht signifikant korreliert, aber dennoch könnte sich der starke Krankheitsbefall von AC Sunset negativ auf die Ausbildung der Achänen ausgewirkt haben. AC Sunset zeigte speziell in Göttingen einen hohen Schalenanteil. Der Schalenanteil war, wie auch Knowles (1969) berichtete, hoch signifikant mit dem Ölgehalt negativ korreliert. Dies zeigt, dass die kanadische Sorte weniger an die klimatischen Bedingungen in Deutschland angepasst ist.

Die Selektion in der Stammbaummethode führte in allen drei Kreuzungen zu gesünderen Linien mit weniger Köpfchenfäule. Bei diesem wichtigen Merkmal war deutlich ein Selektionserfolg zu beobachten. Bei dem ebenfalls im Vordergrund stehenden Merkmal Ölgehalt ließen sich erste Erfolge der 
Selektion in zwei der drei Kreuzungen nachweisen, was die Möglichkeit zeigt, durch die Selektion in der Stammbaummethode Linien mit höheren Ölgehalten zu entwickeln. Interessant sind die Linien, die sowohl einen höheren Ölgehalt als auch geringere Krankheitsanfälligkeit aufweisen. Bei Betrachtung der Daten der einzelnen Linien (dargestellt im Anhang Tab. A XIV - Tab. A XVI), finden sich 22 Linien in der Kreuzung 1 und 18 Linien in der Kreuzung 2, die Verbesserungen gegenüber der Kontrolle in beiden Merkmalen zeigten. Es gab sogar in der ersten Kreuzung zwei und in der zweiten Kreuzung drei Linien, die einen höheren Ölgehalt erzielten und gleichzeitig geringer mit Köpfchenfäule befallen waren als der jeweils bessere Elter. Die Selektionserfolge hängen von der Kreuzung ab, denn in der dritten Kreuzung gab es nur drei Linien, die in beiden Merkmalen besser waren als die Kontrolle.

Bei den Krankheiten Alternaria-Blattflecken und Fusarium wurde kein signifikanter Selektionserfolg nachgewiesen. Fusarium trat in den Jahren der Selektion 2004 und 2005 nicht auf, sodass dies den fehlenden Selektionserfolg erklärt. Beim EP-Ertrag und dem TKG zeigten sich Erfolge, die jedoch nicht in allen Kreuzungen beobachtet werden konnten.

Problematisch war es, Saatgut gleicher Qualität für die Kontrollen zu erstellen, da sich verschiedene Umweltbedingungen besonders während der Reife- und Erntezeit auf die Saatgutqualität auswirken. Bei Alternaria carthami handelt es sich hauptsächlich um ein samenbürtiges Pathogen, das sich auf dem Feld unter bestimmten Witterungsbedingungen schnell ausbreitet (Mündel et al. 1997). Fusarium oxysporum f. sp. carthami überdauert sowohl im Boden als auch in den Achänen (Kaffka und Kearny 1998). Das Saatgut für die Kontrollen wurde im Gewächshaus erzeugt, wo kein Befall mit Alternaria-Blattflecken oder Fusarium beobachtet wurde. So war das Saatgut der Kontrollen möglicherweise weniger belastet als das der Linien und folglich nur bedingt für die Voraussage eines Selektionserfolges anwendbar. Von der Erzeugung des Kontrollsaatgutes auf dem Feld wurde abgesehen, da die Überlebensraten im Freiland meist geringer als im Gewächshaus sind und sowohl Fremdbefruchtung als auch natürliche Selektion die Populationszusammensetzung der Kontrolle möglicherweise verändert hätten.

Das Saatgut der Eltern stammte, wie das der Linien, vom Feld. Daher können die Eltern mit den Linien verglichen werden. Bei dem Merkmal AlternariaBlattflecken waren in allen drei Kreuzungen zahlreiche Linien geringer befallen 
als das Elternmittel (vergleiche Abb. 18). Es gab jedoch keine signifikanten Unterschiede zwischen der Kontrolle und dem Mittel der Eltern. Bei dem Merkmal Fusarium wurden in den ersten beiden Kreuzungen signifikante Unterschiede festgestellt. Doch es gab nur wenige Linien in den drei Kreuzungen, die geringeren Befall zeigten (Abb. 18).

Anhand der Daten der einzelnen Linien, der Eltern und der Kontrolle lassen sich Rückschlüsse auf die Vererbung eines Merkmals ziehen. Bei dem Befall mit Alternaria-Blattflecken war in allen drei Kreuzungen keine Linie so gut wie der bessere Elter und keine Linie schlechter als der stark befallene Elter. Dies deutet darauf hin, dass es sich um eine polygene Vererbung handelt, da bei monogener Vererbung Linien mit einem gleichen Befall wie bei den Eltern hätten auftreten müssen.

Bei den Merkmalen EP-Ertrag, TKG und Schalenanteil könnten epistatische Effekte vorliegen. Ohne Epistasie und ohne Selektion sollte der Mittelwert aller Linien einer Kreuzung dem Mittelwert der Eltern entsprechen (Engqvist und Becker 1991). In den Kreuzungen 1 und 3 wiesen die unselektierten Linien (Kontrolle) einen höheren EP-Ertrag auf als die beiden Eltern.

Bei dem Merkmal TKG zeigte die Kontrolle in der Kreuzung 2 ein geringeres TKG als das Elternmittel. Auch dies deutet auf Epistasie hin. Die ausgewählten Eltern könnten möglicherweise günstige Genkombinationen aufweisen, die bei der Rekombination verloren gingen. Häufig sind infolge von zahlreichen, vorangegangenen Selektionszyklen günstige Genkombinationen im Elternmaterial etabliert (Engqvist und Becker 1991). Durch die Selektion wurde eventuell der negative Effekt in den Linien aufgehoben, sodass eine große Anzahl Linien besser als die Kontrolle waren.

Ramachandram und Goud (1981) berichteten von einer additiven Genwirkung beim Ölgehalt. In den ersten beiden Kreuzungen lagen die Kontrollen nah am Elternmittel. Unerwartet war der hohe Ölgehalt der Kontrolle in der Kreuzung 3 im Vergleich zu den Eltern.

Bei den Linien handelte es sich vermutlich nicht um reine, homozygote Linien, da im ersten Jahr keine Maßnahmen gegen die Fremdbefruchtung vorgenommen wurden und im zweiten Jahr nur die Auskreuzung zwischen den Kreuzungen vermieden wurde. Die innerhalb der Kreuzungen konnte nicht verhindert werden. Das Elternsaatgut stammte von geselbsteten Pflanzen und 
war daher vollständig homozygot. Ebenso waren die Pflanzen in den Kontrollen vermutlich homozygot, da das Saatgut im Gewächshaus erzeugt wurde, sodass eine Fremdbefruchtung durch Insekten größtenteils ausgeschlossen werden konnte. Für Merkmale, die durch Heterosis beeinflusst werden, sind die Kontrollen möglicherweise nicht mit den Linien vergleichbar. Dieses Thema wird später genauer diskutiert. Für die Entwicklung homozygoter Linien müssten besondere Maßnahmen ergriffen werden (siehe Kapitel 4).

Der unterschiedliche und teilweise sehr geringe Feldaufgang stellte bei der Versuchsauswertung ein Problem dar. Durch Krankheiten wie AlternariaBlattflecken und Köpfchenfäule werden nicht vollständig entwickelte Achänen ausgebildet, die die geringe Keimfähigkeit begründen könnten. Erstaunlich war der zum Teil sehr starke Befall mit Krankheiten, da es sich bei Saflor um eine, nach langer Zeit, wieder eingeführte Kulturart handelt. So könnte angenommen werden, dass es weniger Erreger in deutschen Anbauregionen gibt, die Saflor befallen. Der Erreger der Köpfchenfäule (Botrytis cinerea) ist jedoch nicht spezifisch für die Färberdistel, sondern befällt auch andere Kulturarten wie Raps und Sonnenblume. Bei samenbürtigen Erregern, wie Alternaria carthami, sollte darauf geachtet werden, dass das eingeführte Saatgut frei von Befall ist. Möglicherweise sollte das Saatgut vor Beginn der Studien einige Generationen im Gewächshaus vermehrt werden.

Um einen gleichmäßigen Feldaufgang zu erhalten, wurden vor der Aussaat Keimtests durchgeführt, nach denen die Aussaatstärke bestimmt wurde. In Hohenheim war der Feldaufgang in 2006 dennoch sehr gering, was den Vergleich mit anderen Standorten problematisch gestaltete. Auch an den anderen Standorten zeigte sich bei den einzelnen Linien unterschiedlicher Feldaufgang. Besonders für die Ertragsdaten ist ein gleichmäßiger Feldaufgang relevant. Der Parzellenertrag war aufgrund der sehr unterschiedlichen Anzahl Pflanzen in einer Parzelle nicht vergleichbar. Auch der Einzelpflanzenertrag war stark von der Anzahl Pflanzen in einer Parzelle abhängig, je weniger Pflanzen desto höher der Einzelpflanzenertrag. Um den Ertrag der Linien zu vergleichen, wurde daher der bereinigte Einzelpflanzenertrag berechnet, der von der Anzahl Pflanzen in einer Parzelle unabhängig ist. 


\subsubsection{Bewertung der Methode der natürlichen Selektion}

Obwohl die Ramschgenerationen nur zwei Zyklen natürlicher Selektion unterlagen, führte die natürliche Selektion zu gesünderen Beständen. Der Befall mit Köpfchenfäule nahm in allen drei Kreuzungen an allen Herkunftsorten signifikant ab. In anderen Studien wurde ebenfalls eine Reduktion der Krankheitsanfälligkeit durch natürliche Selektion festgestellt. Beispielsweise stellten Paillard et al. (2000 a, b) bei Untersuchungen mit Winterweizen eine Veränderung im Befall mit Mehltau (Erysiphe graminis) nach zehn Jahren natürlicher Selektion fest. Es war zu erwarten, dass gesündere Pflanzen bevorzugt werden, bemerkenswert ist, dass sich bereits nach zwei Zyklen natürlicher Selektion signifikante Unterschiede im Befall mit Köpfchenfäule zeigten. Beim Befall mit Alternaria-Blattflecken und Fusarium konnten keine signifikanten Verbesserungen festgestellt werden, was durch die geringe Anzahl Selektionszyklen erklärt werden könnte.

Bei dem Befall mit Fusarium gab es zwischen den Ramschherkünften Göttingen und Wilmersdorf Unterschiede, die in den Kreuzungen jedoch nicht einheitlich waren. Es wurde eine unterschiedlich starke Belastung des Saatgutes mit Fusarium vermutet. Bei anschließenden Untersuchungen wurden jedoch bei keiner Saatgutprobe Fusariumerreger gefunden.

Bei einem weiteren wichtigen Merkmal, dem Ölgehalt, konnte kein Einfluss der natürlichen Selektion festgestellt werden. Es war jedoch auch nicht unbedingt zu erwarten, dass Pflanzen mit einem höheren Ölgehalt durch natürliche Selektion begünstigt sind.

Beachtenswert war der Anstieg im Ertrag. Ähnliche Ergebnisse fand Pathirana (1995) bei Studien mit Sesam. Er zeigte, dass die weniger aufwändige Ramschmethode gleichen Erfolg wie die Stammbaummethode für Merkmale wie den Ertrag erzielte.

Die morphologischen und phänologischen Merkmale standen in der Gewichtung hinter den soeben beschriebenen agronomischen Merkmalen. Dennoch war es interessant einen Anstieg im TKG zu beobachten. Größere Samen sind in der Geschichte der Kulturpflanzen ein typisches Merkmal der Domestikation (Horneburg 2003). Möglicherweise waren in der vorliegenden Arbeit andere mit der Achänengröße korrelierte, nicht untersuchte Eigenschaften für diese Entwicklung entscheidend. 
Die natürliche Selektion führte zu kürzeren Pflanzen, was allerdings in den Kreuzungen nicht einheitlich und nicht signifikant war. Studien an Linsen (Haddad und Muehlbauer 1981), an Weizen (Tee und Qualset 1975) und an Sojabohnen (Empig und Fehr 1971) zeigten dagegen, dass größere Pflanzen in der natürlichen Selektion bessere Konkurrenzeigenschaften aufwiesen.

Der Vergleich zwischen der Kontrolle und den Herkünften ist nicht unproblematisch. Horneburg (2003) beschrieb, dass die Vermehrung im Gewächshaus als eine spezielle Umwelt mit entsprechenden Einflüssen auf das Saatgut anzusehen sei. Auch das Saatgut verschiedener Herkünfte kann nur bedingt verglichen werden, da verschiedene Umweltbedingungen in der Reife- und Erntezeit die Saatgutqualität beeinträchtigen können. Die Keimfähigkeit des Saatgutes und der Aufgang in den Parzellen stellten hier, wie in der Stammbaummethode, Probleme dar. Die Saatgutqualität war sehr unterschiedlich. Trotz Keimprüfung und Berechnung gleicher Aussaatstärke konnte kein gleichmäßiger Aufgang erreicht werden. Durch Vereinzeln sehr eng stehender Bestände wurde versucht eine bessere Vergleichbarkeit zu schaffen. Dies konnte jedoch nur bedingt erfolgen, da in manchen Parzellen der Feldaufgang zu gering war und nicht alle Parzellen auf ein solches Minimum reduziert werden sollten. Für die Erfassung der Ertragsdaten wurde, wie in der Stammbaummethode, deshalb der bereinigte EP-Ertrag gewählt.

Die Standorte der Auslese waren in der Bodenbeschaffenheit und Niederschlagsmenge sehr unterschiedlich. Niederschläge während der Blüteund Reifezeit führen vermehrt zu Krankheiten. So war zu beobachten, dass auf den nährstoffreichen Böden in Göttingen die Pflanzen größer wurden, aber meist durch stärkeren Krankheitsbefall belastet waren. Eine stärkere natürliche Selektion bei dem Krankheitsbefall war an diesem Standort jedoch nicht zu beobachten. David et al. (1997) beschrieben bei Studien mit Weizen, dass der Ort der Auslese Auswirkungen auf die Populationsentwicklung hatte. Eine divergierende Entwicklung ist zu erwarten, wenn sehr heterogene Populationen der Auslese an mehreren Orten unterliegen (Jana und Khangura 1986). Standortspezifische Anpassungen durch die natürliche Selektion waren in der vorliegenden Arbeit nicht deutlich zu erkennen. Es handelte sich jedoch nur um einen kurzen Versuchszeitraum. Die Ramschherkünfte unterlagen nur zwei Generationen natürlicher Selektion, die der Kreuzungen 1 und 3 des Herkunftsortes Hohenheim sogar nur einer Generation. Daher kann hier keine 
genaue Aussage gemacht werden, ob es standortspezifische Anpassungen geben könnte. Die Versuche müssten hierfür über mehrere Jahre mehrortig durchgeführt werden.

Auch in der Methode der natürlichen Selektion muss die Fremdbefruchtung beachtet werden. Dies wird in Kapitel 4 genauer erläutert.

\subsubsection{Bewertung der Einkornramschmethode}

Der Grundgedanke der Einkornramschmethode ist, dass jede F2-Pflanze später mit einer Linie vertreten sein soll. Die natürliche Selektion soll hier verhindert werden. Umweltbedingungen sind daher ohne Bedeutung, sodass diese Methode zur Beschleunigung beispielsweise im Gewächshaus durchgeführt werden kann.

Fernández-Martínez et al. (1986) untersuchten die Einkornramschmethode bei Saflor. Grundlage dafür war eine Kreuzung zwischen einer gut angepassten Sorte und einer Sorte mit hohem Ölgehalt. Zahlreiche F4- und F5-Linien wiesen höhere Erträge und Ölgehalte als der bessere Elter auf. Fernández-Martínez et al. (1986) kamen zu dem Schluss, dass diese Ergebnisse und die große Ersparnis an Zeit und Aufwand die Anwendung der Einkornramschmethode in der Saflorzüchtung als „ausgesprochen wünschenswert“ erscheinen lassen.

Bei einem Vergleich zwischen der Ramschmethode und der Einkornramschmethode bei Linsen berichteten Haddad und Muehlbauer (1981), dass die Einkornramschmethode eine größere genetische Variation hervorbringe und sich für die Linsenzüchtung gut eigne.

In der vorliegenden Arbeit konnten nur Versuchsdaten des Standortes Göttingen ausgewertet werden. In Hohenheim war der Aufgang aus ungeklärten Gründen sehr schlecht. Ein generelles Problem war die Saatgutverfügbarkeit, da die Saflorpflanzen im Gewächshaus nur wenige Achänen ausbildeten, sodass es an beiden Standorten keine Wiederholungen gab. Pro Linie und Elter wurde eine Doppelreihe mit 20 Achänen ausgesät. Folglich war der Versuchsfehler in den Daten sehr hoch. Die Vergleiche der Linien mit dem Elternmittel und dem besseren Elter erwiesen sich als sehr ungenau, da die Daten der Eltern jeweils nur von einer Doppelreihe aufgenommen wurden. Die Ergebnisse zum Selektionserfolg waren in den einzelnen Kreuzungen sehr unterschiedlich. 
Aus diesen Gründen werden die Ergebnisse der Einkornramschmethode im Folgenden nicht weiter berücksichtigt.

Um die Saatgutverfügbarkeit zu erhöhen, wäre es sinnvoll gewesen, F6Pflanzen im Freiland anzubauen und zu selbsten.

\subsubsection{Schlussfolgerung}

Der prinzipielle Unterschied zwischen der Stammbaum- und der Ramschmethode besteht in der Selektion in frühen Generationen. Ist die Selektion von Einzelpflanzen in den spaltenden Generationen wirksam, kann die Stammbaummethode von Vorteil sein. Der Selektionserfolg hängt vom Merkmal ab. Im Krankheitsbefall müssen deutlich sichtbare Differenzierungen auftreten, damit die visuelle Selektion in der Stammbaummethode erfolgreich ist. In der Ramschmethode kann hier die natürliche Selektion genutzt werden. Ein Selektionserfolg bei Inhaltsstoffen, wie die Erhöhung des Ölgehaltes, ist durch die natürliche Selektion jedoch nicht zu erwarten. Es hängt von der genetischen Variation im Material, von der Heritabilität des Merkmals und von den Umweltbedingungen ab, welche Methode zu bevorzugen ist (Becker 1993). Salas und Friedt (1995) untersuchten die Stammbaummethode bei Lein. Sie kamen zu dem Schluss, dass eine Selektion in frühen Generationen bei hoch heritablen Merkmalen wie dem Ölgehalt sinnvoll erscheint, auf weniger heritable Merkmale wie den Ertrag jedoch erst in späteren Generationen selektiert werden sollte.

Ob die Stammbaummethode in frühen Generationen zur effizienteren Selektion führt, hängt unter anderem von der Wirkungsweise der Allele ab. Sind die günstigen Allele rezessiv, können sie nur in homozygoten Pflanzen entdeckt werden, die erst in späteren Selbstungsgenerationen gehäuft erscheinen. Sind die Allele für ein günstiges Merkmal dominant, können sie schon in frühen Generationen in heterozygoten Pflanzen entdeckt werden und durch die schrittweise Selektion in der Stammbaummethode können aus diesen Pflanzen homozygote Linien mit seltenen Genkombinationen entwickelt werden. Die Selektion von Einzelpflanzen an nur einem Ort bereitet Probleme. Die genetischen Unterschiede zwischen Einzelpflanzen werden häufig durch Umwelteinflüsse überdeckt (Becker 1993). Aus diesem Grund wurde 2004 in der Stammbaummethode zunächst der durchschnittliche Ölgehalt der F3-Linien 
errechnet. Anschließend wurden von den besten F3-Linien Einzelpflanzen zufällig weitergeführt.

Die Zeitspanne in der vorliegenden Arbeit war gering für die Untersuchung von Züchtungsmethoden. In der Ramschmethode gab es zwei Zyklen natürlicher Selektion. Es wurden noch keine Einzelpflanzen selektiert. Die untersuchten Generationen stellen jedoch den entscheidenden Unterschied zwischen der Stammbaum- und der Ramschmethode dar und in beiden Methoden konnten Selektionserfolge in verschiedenen Merkmalen beobachtet werden.

Sowohl durch die natürliche Selektion als auch durch die visuelle Selektion in der Stammbaummethode wurde die Krankheitsanfälligkeit für Köpfchenfäule in allen drei Kreuzungen verringert. Linien mit höherem Ölgehalt konnten dagegen nur in der Stammbaummethode entwickelt werden. Ertragssteigerungen wurden durch die natürliche Selektion deutlicher erreicht.

Die natürliche Selektion ist weniger arbeitsintensiv, platzsparender auf dem Feld und erzielt gute Erfolge. Für die Züchtung von Saflor erscheint es somit sinnvoll, zuerst die F2- und F3-Generationen als Ramsch anzubauen, um durch die natürliche Selektion eine weniger krankheitsanfällige Population mit höherem Ertrag zu erhalten. Anschließend sollten Nachkommen von Einzelpflanzen weitergeführt und selektiert werden. Hier steht dann neben Krankheitsanfälligkeit und Ertrag vor allem der Ölgehalt im Vordergrund. 


\section{Fremdbefruchtung und Heterosis}

\subsection{Einleitung}

Die Angaben über die Fremdbefruchtungsrate bei Saflor variieren in der Literatur stark. Meist wird er als überwiegender Selbstbefruchter eingestuft. Bei Knowles (1969) wurde eine Fremdbestäubung unter 10\% erwähnt. Dajue und Mündel (1996) beschrieben, dass je nach Umweltbedingung auch über 50\% möglich seien. Scheibe und Yekta (1934) beschrieben Saflor als Fremdbefruchter. Später fand Scheibe (1939) bei Isolierungsversuchen heraus, dass neben der "gewöhnlichen Fremdbefruchtung auch in geringem Umfang Selbstbefruchtung" möglich sei. Einheitlich wird in der Literatur beschrieben, dass für die Fremdbefruchtung Insekten, nicht Wind, verantwortlich sind (Scheibe 1939, Knowles 1989, Dajue und Mündel 1996, Weiss 2000).

Die Bestäubung geschieht gewöhnlich, wenn sich der Griffel durch die fünf, zu einer Röhre verwachsenen, Antheren schiebt (Abb. 20). Eine unbestäubte, gestreckte Narbe bleibt für einige Tage empfänglich. Insekten suchen die Blüten wegen des Nektars und der Pollen auf und erhöhen die Fremdbefruchtungsrate (Dajue und Mündel 1996).

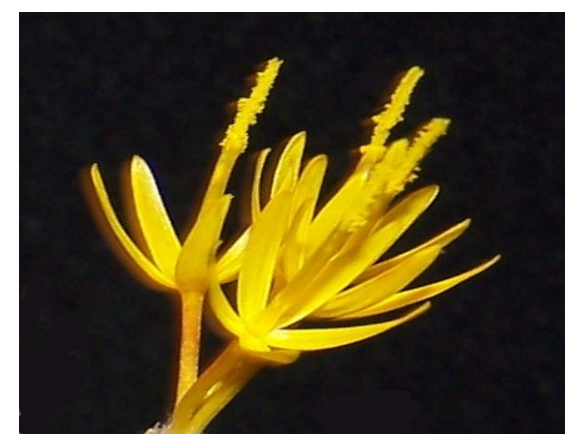

Abb. 20: Saflorblüten mit herausgeschobenen Griffeln und Pollen

Für die Züchtung von Saflor ist die Fremdbefruchtungsrate von großer Bedeutung. Bei der Entwicklung von Linien müssten bei hoher Fremdbefruchtungsrate Maßnahmen, wie Isolation oder Selbstung, gegen die Auskreuzung ergriffen werden. 
Besonders wichtig ist die Kenntnis über die Fremdbefruchtungsrate bei der Beurteilung des Selektionserfolges von Merkmalen, die von Heterosis beeinflusst werden können. Ein Anstieg des Ertrages könnte in offen abgeblühten Populationen auf dem Vorteil der Heterozygotie beruhen.

In der vorliegenden Arbeit blühten die Pflanzen bei der Untersuchung der Ausleseverfahren offen ab. Die Fremdbefruchtung und die Heterosis wurden daher parallel zu den Ausleseverfahren untersucht, um mögliche Effekte auf die Ergebnisse der Stammbaummethode und der Methode der natürlichen Selektion einschätzen zu können. Dabei wurde zwischen der Fremdbefruchtung zwischen Pflanzen innerhalb derselben Parzelle und Fremdbefruchtung zwischen benachbarten Parzellen unterschieden. 


\subsection{Material und Methoden}

\subsubsection{Untersuchungen zur Fremdbefruchtung}

Für die Untersuchungen der Fremdbefruchtung wurde geselbstetes Saatgut der unbestachelten Sorte Sabina und des bestachelten Genotyps CR1 verwendet. Beide blühen gelb. Die Fremdbefruchtung zwischen den Parzellen wurde einjährig, die innerhalb der Parzelle zweijährig untersucht. Die Versuche wurden in Göttingen angelegt. Nach Pahlavani et al. (2004) wurde angenommen, dass das Merkmal „bestachelt“ vollständig dominant gegenüber dem Merkmal „unbestachelt“ ist.

\section{Fremdbefruchtung zwischen Parzellen}

In 2004 wurde eine $10 \mathrm{~m}^{2}$ große Parzelle mit einer Aussaatstärke von 40 Achänen $/ \mathrm{m}^{2}$ der Sorte Sabina, umgeben von Parzellen mit bestachelten Pflanzen, angebaut. Je zehn Pflanzen wurden aus beiden Rändern (Rand 1 und Rand 2) und der Mitte der unbestachelten Parzelle geerntet und 45 Achänen pro Pflanze im nächsten Jahr auf dem Feld ausgesät. Aus dem Verhältnis der bestachelten Nachkommen zu den unbestachelten wurde die Fremdbefruchtungsrate ermittelt.

Der Versuch wurde 2004 neben einem Saflorfeld angelegt, sodass auf Rand 1 eine Parzelle CR1 und das Saflorfeld folgte, auf Rand 2 nur eine Parzelle CR1 (Abb. 21).

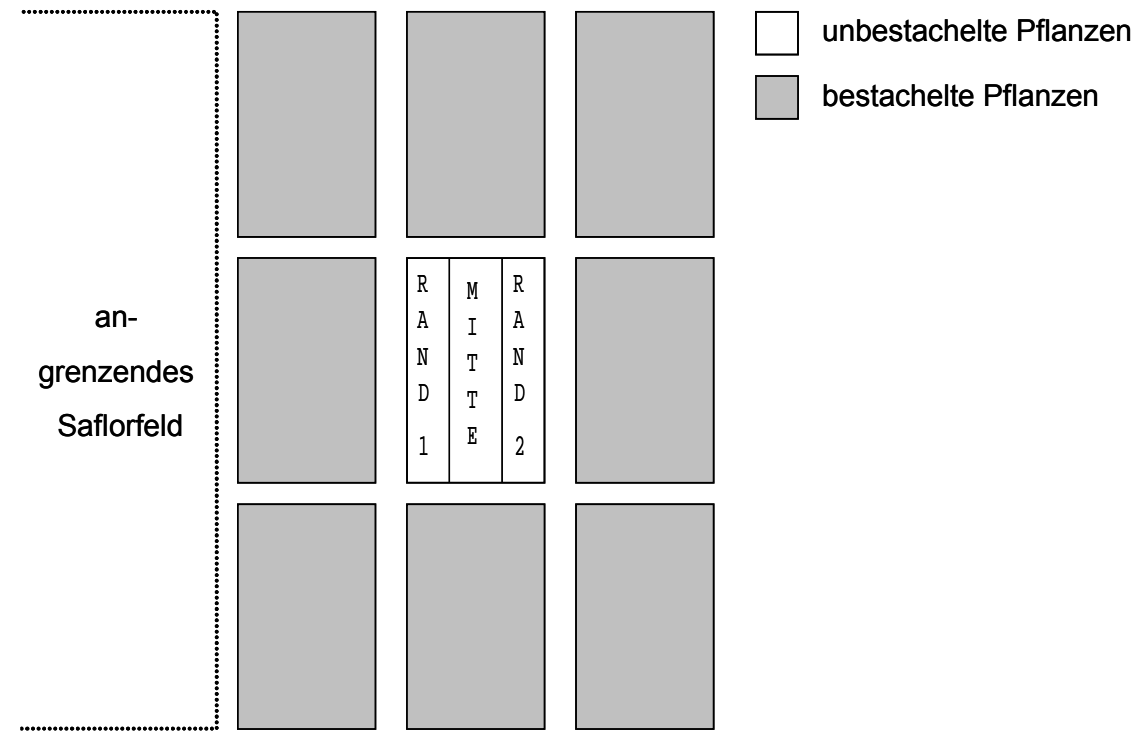

Abb. 21: Versuchsanlage zur Untersuchung der Fremdbefruchtung zwischen Parzellen 2004 


\section{Fremdbefruchtung innerhalb einer Parzelle}

Je 35 Achänen von Sabina und 315 Achänen von CR1 wurden gemischt und in einer $10 \mathrm{~m}^{2}$ großen Parzelle angebaut. In 2004 wurde der Versuch neben anderen Saflorversuchen angelegt, 2005 wurde die Parzelle isoliert.

Der Anteil aufgegangener unbestachelter Pflanzen am Gesamtaufgang wurde gezählt. Je zehn Pflanzen Sabina wurden zufällig geerntet. In 2005 wurden 50 Nachkommen pro Pflanze aus 2004 im Gewächshaus und in 2006135 Nachkommen pro Pflanze aus 2005 auf dem Feld ausgesät. Der Anteil bestachelter und unbestachelter Nachkommen wurde ermittelt. Die Fremdbefruchtung von unbestachelten Pflanzen untereinander in einer Parzelle wurde wie folgt berücksichtigt:

$$
\begin{array}{ll}
y^{\prime}=\frac{100}{x} \cdot y, \text {, wobei } \\
y^{\prime} & : \text { Fremdbefruchtungsrate } \\
x & : \text { Anteil der bestachelten Pflanzen in einer Parzelle } \\
y & : \text { Anteil bestachelter Nachkommen }
\end{array}
$$

\subsubsection{Untersuchungen zur Heterosis}

In 2005 wurden jeweils zwei Eltern (Kreuzung 1: AC Sunset und CR1, Kreuzung 2: Saffire und CR1, Kreuzung 3: Sabina und AC Sunset) in einer ein $\mathrm{m}^{2}$ großen, isolierten Parzelle in Göttingen und in Wilmersdorf angebaut. Die offen abgeblühten Pflanzen wurden parzellenweise geerntet. Je nach Fremdbefruchtungsrate handelte es sich dabei teilweise um F1-Saatgut.

In einer Blockanlage wurden 2006 die Nachkommen aus 2005, die Eltern und eine Mischung der Eltern für jede Kreuzung in Göttingen in zweifacher Wiederholung angebaut. Die Merkmale Pflanzenlänge, TKG, EP-Ertrag (bereinigter Einzelpflanzenertrag) und Ölgehalt wurden, wie in der Stammbaummethode beschrieben (Kapitel 3), untersucht.

Wegen mangelnden Aufgangs in 2005 konnte die Kreuzung 2 nur von dem Herkunftsort Göttingen und die Kreuzung 3 nur aus Wilmersdorf geprüft werden. 


\subsection{Ergebnisse}

\subsubsection{Untersuchungen zur Fremdbefruchtung}

\section{Fremdbefruchtung zwischen Parzellen}

In der Mitte der untersuchten Parzelle kam es nur zu relativ geringer Fremdbefruchtung. Zwischen den beiden Rändern waren deutliche Unterschiede festzustellen. Im Rand 1, an den ein Saflorfeld grenzte, wurden mit 9,7\% wesentlich geringere Fremdbefruchtungsraten beobachtet als im Rand 2, auf den nur eine Parzelle bestachelter Pflanzen folgte (Tab. 44). Die Daten der je zehn untersuchten Pflanzen finden sich im Anhang Tab. A XVIII.

Tab. 44: Fremdbefruchtung (\%) zwischen Parzellen 2004

\begin{tabular}{lcccc}
\hline & $\begin{array}{c}\text { Anzahl } \\
\text { Nachkommen }\end{array}$ & $\begin{array}{c}\text { bestachelte } \\
\text { Nachkommen }\end{array}$ & $\begin{array}{c}\text { Fremdbefruchtung } \\
\mathbf{( \% )}\end{array}$ & $\begin{array}{c}\text { Spannweite } \\
\mathbf{( \% )}\end{array}$ \\
\hline Rand 1 & 353 & 33 & 9,7 & $5,7-17,1$ \\
Mitte & 382 & 25 & 6,5 & $0,0-11,5$ \\
Rand 2 & 259 & 47 & 18,1 & $8,3-33,3$ \\
\hline
\end{tabular}

\section{Fremdbefruchtung innerhalb einer Parzelle}

Die Fremdbefruchtungsrate innerhalb einer Parzelle war wesentlich höher als die zwischen Parzellen. In den beiden Versuchsjahren 2004 und 2005 waren sehr unterschiedliche Ergebnisse zu beobachten. Auch innerhalb der zehn untersuchten Pflanzen pro Jahr war die Spannweite sehr groß (Tab. 45). Die Fremdbefruchtungsraten der einzelnen Pflanzen sind im Anhang Tab. A XIX dargestellt.

Im Jahr 2004 war lag die Fremdbefruchtung hauptsächlich zwischen 50\% und $65 \%, 2005$ war sie dagegen deutlich niedriger. Bei sechs Pflanzen war in diesem Jahr eine Fremdbefruchtungsrate unter $30 \%$ zu beobachten (Abb. 22).

Tab. 45: Fremdbefruchtung (\%) innerhalb einer Parzelle 2004 und 2005

\begin{tabular}{ccccc}
\hline & $\begin{array}{c}\text { Anzahl } \\
\text { Nachkommen }\end{array}$ & $\begin{array}{c}\text { bestachelte } \\
\text { Nachkommen }\end{array}$ & $\begin{array}{c}\text { Fremdbefruchtung } \\
\text { (\%) }\end{array}$ & $\begin{array}{c}\text { Spannweite } \\
\text { (\%) }\end{array}$ \\
\hline 2004 & 372 & 219 & 63,1 & $39,8-87,1$ \\
2005 & 728 & 172 & 29,9 & $17,4-43,7$ \\
\hline
\end{tabular}




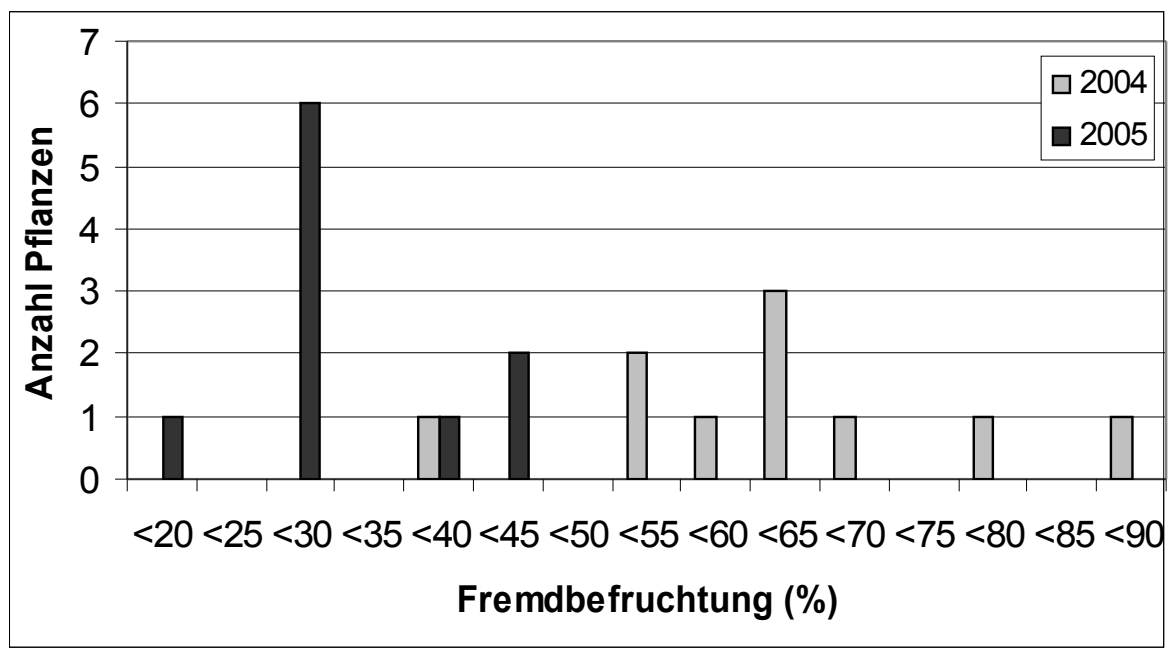

Abb. 22: Verteilung der Fremdbefruchtung (\%) 2004 und 2005

\subsubsection{Untersuchungen zur Heterosis}

Die Parzellen mit den Mischungen der Eltern dienten zur Überprüfung, ob die Nachkommen der offen abgeblühten Parzellen Heterosis aufwiesen. Ohne Heterosis hätten die Werte der Nachkommen denen der Mischungen ähnlich sein müssen.

Beim Ölgehalt wurde im Mittel keine deutliche Auswirkung der Heterosis beobachtet. Die Ergebnisse variierten in den Kreuzungen jedoch sehr stark.

Im Mittel waren die Nachkommen um 7,1\% länger als die Mischungen der Eltern. Der EP-Ertrag der Nachkommen war um 9,1\% höher und das TKG der Nachkommen lag im Mittel 10,4\% über dem der Elternmischungen (Tab. 46). 
Tab. 46: Ergebnisse der Untersuchungen zur Heterosis

\begin{tabular}{|c|c|c|c|c|}
\hline & Pflanzenlänge (cm) & Ölgehalt (\%) & EP-Ertrag (g) & TKG (g) \\
\hline \multicolumn{5}{|c|}{ AC Sunset und CR1 } \\
\hline Nachkommen Gö & 95,0 & 18,24 & 17,82 & 3,13 \\
\hline Nachkommen Wi & 105,0 & 17,12 & 17,77 & 3,30 \\
\hline Mischung & 94,0 & 17,03 & 15,68 & 3,09 \\
\hline Mittel Eltern & 92,5 & 19,38 & 16,57 & 3,00 \\
\hline Heterosis Gö (\%) & 1,1 & 7,1 & 13,6 & 1,3 \\
\hline Heterosis Wi (\%) & 11,7 & 0,5 & 13,3 & 6,8 \\
\hline \multicolumn{5}{|l|}{ Saffire und CR1 } \\
\hline Nachkommen Gö & 105,5 & 22,92 & 17,09 & 3,24 \\
\hline Mischung & 95,0 & 18,65 & 15,02 & 2,83 \\
\hline Mittel Eltern & 93,8 & 21,58 & 18,09 & 2,92 \\
\hline Heterosis Gö (\%) & 11,1 & 22,9 & 13,8 & 14,5 \\
\hline \multicolumn{5}{|l|}{ Sabina und Saffire } \\
\hline Nachkommen Wi & 107,5 & 19,01 & 12,84 & 3,21 \\
\hline Mischung & 100,0 & 21,98 & 14,33 & 2,83 \\
\hline Mittel Eltern & 106,3 & 19,31 & 14,26 & 2,92 \\
\hline Heterosis Wi (\%) & 7,5 & $-13,5$ & $-10,4$ & 13,4 \\
\hline \multicolumn{5}{|l|}{ Mittel } \\
\hline Nachkommen & 103,3 & 19,32 & 16,38 & 3,22 \\
\hline Mischungen & 96,4 & 19,22 & 15,01 & 2,92 \\
\hline Mittel Eltern & 97,5 & 20,09 & 16,31 & 2,94 \\
\hline Heterosis (\%) & 7,1 & 0,5 & 9,1 & 10,4 \\
\hline
\end{tabular}

Gö: Göttingen, Wi: Wilmersdorf 


\subsection{Diskussion}

Die Beobachtungen zur Fremdbefruchtung bei Saflor differieren in der Literatur sehr stark. Die Färberdistel wird meist als überwiegender Selbstbefruchter beschrieben. Je nach Umweltbedingungen können aber auch bis zu 50\% Fremdbefruchtung möglich sein (Dajue und Mündel 1996).

Der Umfang der Versuche in dieser Arbeit zur Untersuchung der Fremdbefruchtungsrate und der Heterosis war zeitlich eingeschränkt.

Die Fremdbefruchtungsrate wurde nur an einem Ort über ein beziehungsweise zwei Jahre untersucht. Die Ergebnisse der Fremdbefruchtungsrate innerhalb einer Parzelle 2004 und 2005 waren sehr unterschiedlich. Eine Erklärung hierfür zu finden, ist jedoch ohne weitere Untersuchungen nicht möglich. Verschiedene Umwelteinflüsse, aber auch die isolierte Lage im Jahr 2005 könnten Gründe dafür liefern. Weitere Aspekte wie beispielsweise eine Abhängigkeit der Fremdbefruchtungsrate vom Genotyp oder der Blütenfarbe wurden nicht untersucht. Die Ergebnisse der vorliegenden Arbeit können daher nicht verallgemeinert werden. Um genauere Ergebnisse, beispielsweise über Umwelteinflüsse, zu erhalten, müssten die Versuche über einen längeren Zeitraum mehrortig angebaut und ausgewertet werden. Untersuchungen über die Auswirkung der Köpfchenposition an der Pflanze wären ebenfalls interessant.

Den Einfluss der Umwelt auf die Fremdbefruchtung von Sommerraps stellten Becker et al. (1992) dar. Die Fremdbefruchtung wurde an fünf verschiedenen Orten in Schweden, Dänemark und Norddeutschland untersucht. Sie variierte an den Orten zwischen $12 \%$ und $47 \%$. Zwischen den Blüten an verschiedenen Positionen an der gleichen Pflanze gab es ebenfalls Unterschiede. Die Daten variierten von $11 \%$ an der Spitze des Blütenstandes bis $39 \%$ an der Basis.

Die Fremdbefruchtungsrate kann auch vom Heterozygotiegrad abhängen. Link (1990) fand bei Untersuchungen von Elternlinien und Kreuzungen bis zur F3 heraus, dass bei der Ackerbohne die Fremdbefruchtungsrate mit zunehmender Heterozygotie sank.

Interessant wären Untersuchungen, ob auch bei Saflor die Fremdbefruchtungsrate vom Heterozygotiegrad abhängt. In dieser Arbeit wurde nur die Fremdbefruchtungsrate der homozygoten Sorte Sabina ermittelt. 
Möglicherweise wäre die Fremdbefruchtungsrate bei heterozygoten Genotypen niedriger. Erkenntnisse darüber wären für die verschiedenen Züchtungsmethoden interessant, da beispielsweise bei der Stammbaummethode und bei der natürlichen Selektion die Pflanzen anfangs noch stärker heterozygot sind. Maßnahmen zur Verhinderung der Fremdbefruchtung könnten dann angepasst oder in späteren Generationen verstärkt vorgenommen werden.

Möglicherweise hatte die Fremdbefruchtung einen Einfluss auf den Ölgehalt. Letchworth und Lambert (1998) berichteten bei Studien mit Mais von Unterschieden im Ölgehalt bei offen abgeblühten und geselbsteten Körnern. Sie kamen zu dem Schluss, dass für genaue Bestimmungen der Ölgehalt von geselbsteten Körnern untersucht werden solle. Auch bei Studien mit Raps wurde ein Einfluss des Pollens auf den Ölgehalt festgestellt (Hom 2004).

Um die Aussage von Pahlavani et al. (2004), das Merkmal „bestachelt“ sei vollständig dominant gegenüber dem Merkmal „unbestachelt“, zu überprüfen, sollte in dieser Arbeit das Spaltungsverhältnis bei Nachkommen von geselbsteten, bestachelten F1-Pflanzen untersucht werden. Aufgrund sehr schlechten Aufgangs konnte der Versuch jedoch nicht ausgewertet werden. Baydar und Gökmen (2003) benutzten bei der Erzeugung von Hybridsaatgut jedoch ebenfalls die Bestachelung als Marker und beschrieben auch, dass das Merkmal „bestachelt“ durch ein einziges dominantes Gen vererbt wird.

Problematisch war bei den Untersuchungen zur Fremdbefruchtung die Aufgangsrate der Nachkommen. Es wurde nicht festgestellt, ob die Keimfähigkeit von gekreuztem Saatgut der von selbstbefruchtetem Saatgut gleicht. Nur ungefähr $65 \%$ der ausgesäten Nachkommen sind aufgegangen. Es konnte nicht ermittelt werden, ob die nicht gekeimten Achänen bevorzugt gekreuzten oder selbstbefruchteten Ursprungs waren. Im Bezug auf die vorliegende Arbeit war dies jedoch weniger relevant, da in den Versuchen nur die Fremdbefruchtungsrate bezogen auf die tatsächlich aufgegangenen Pflanzen von Interesse war.

Für die Resultate der vorliegenden Arbeit und auch für zukünftige Versuche können aus den Untersuchungen zur Fremdbefruchtung in dieser Arbeit Rückschlüsse gezogen werden. Insgesamt war die Fremdbefruchtung relativ hoch, sodass bei der Entwicklung von Linien darauf geachtet werden muss, durch Isolierungen oder Selbstungen die Auskreuzung zu verhindern. Eine 
weitere Möglichkeit wäre nur die in der Mitte stehenden Pflanzen in der Parzelle zu ernten.

Die Fremdbefruchtung zwischen Parzellen war deutlich niedriger als die innerhalb einer Parzelle. Aufgrund dieser Ergebnisse wurden die Versuchsanlagen 2005 so angepasst, dass zwischen den Kreuzungen in der Stammbaummethode Ränder mit Pflanzen der jeweilig gleichen Kreuzung angelegt wurden. Die Auskreuzung zwischen den Doppelreihen der Kreuzungen zu verhindern wäre sehr schwierig gewesen. Es hätten Selbstungen vorgenommen werden müssen, die jedoch nicht genügend Saatgut für den Vergleichsanbau 2006 geliefert hätten. Die Parzellen der natürlichen Selektion wurden weitgehend isoliert angebaut. So wurde eine Auskreuzung zwischen den Kreuzungen verhindert, innerhalb der Ramschparzelle konnte sie nicht verhindert werden. Bei den F5-Linien in der Stammbaummethode und den Ramschherkünften der natürlichen Selektion wurde folglich nicht der Homozygotiegrad eines Selbstbefruchters in gleicher Generation erreicht.

Besonders bei dem Vergleich der aus dem Gewächshaus stammenden Kontrollen mit den F5-Parzellen der Stammbaummethode und der natürlichen Selektion stellt sich die Frage, ob die beobachteten Merkmalsdaten von Heterosis beeinflusst sein können.

Das Saatgut für die Untersuchung der Heterosis wurde durch offenes Abblühen hergestellt. Aufgrund der Fremdbefruchtung handelte es sich bei den Nachkommen teilweise um F1-Pflanzen. Durch den Vergleich mit den Elternmischungen sollte beurteilt werden, ob diese Nachkommen Heterosis aufwiesen. Der Versuch wurde nur einjährig an einem Standort durchgeführt. Für genauere Aussagen, wie zum Beispiel über Effekte der Umwelt, müssten weitere Versuche an mehreren Orten und über mehrere Jahre ausgewertet werden.

Ziel der hier dargestellten Versuche war es, mögliche Auswirkungen der Heterosis auf die Ergebnisse der Ausleseverfahren zu untersuchen. Die Pflanzen in der Stammbaumethode und in der Methode der natürlichen Selektion sind offen abgeblüht. Fremdbefruchtung konnte nicht vollständig verhindert werden. Besonders bei der natürlichen Selektion könnte der Anstieg 
des Ertrages auf dem Vorteil heterozygoter Pflanzen beruhen und nicht auf der natürlichen Selektion.

In den Untersuchungen wurde für den Ölgehalt im Mittel nur ein geringes Ausmaß an Heterosis gefunden. Bei den Merkmalen EP-Ertrag und TKG wurde im Mittel ein Anstieg von $9,1 \%$ und $10,4 \%$ bei den Nachkommen gegenüber den Elternmischungen beobachtet. Je nach Fremdbefruchtungsrate könnten daher die Ergebnisse dieser Merkmale in der Stammbaummethode und in der Methode der natürlichen Selektion von Heterosis beeinträchtigt worden sein. Möglicherweise kann der Anstieg des Ertrages und des TKG nicht ausschließlich auf die natürliche Selektion zurückgeführt werden. Für genauere Aussagen wäre jedoch die Kenntnis notwendig, wie hoch die Fremdbefruchtungsrate an den einzelnen Standorten in den Versuchsjahren war. 


\section{Schlussbetrachtung}

Bei der Untersuchung der verschiedenen Züchtungsmethoden wurde festgestellt, dass es möglich ist, Linien mit geringerer Anfälligkeit für Köpfchenfäule und höherem Ölgehalt zu entwickeln. Durch natürliche Selektion wurden mit relativ geringem Arbeitsaufwand gute Erfolge erzielt. Der Befall mit Köpfchenfäule sank und der Ertrag stieg. Demnach ist es in der Saflorzüchtung sinnvoll, zunächst die F2- und F3-Generationen der natürlichen Selektion zu unterziehen. Erst danach sollten Nachkommen einzelner Pflanzen geprüft und selektiert werden. Hier sollte zunächst, neben der geringeren Krankheitsanfälligkeit, der Ölgehalt im Vordergrund stehen, da durch die natürliche Selektion keine Steigerung des Ölgehaltes erreicht wurde.

Bei der Selektion auf höheren Ölgehalt bietet NIRS eine gute Möglichkeit, eine große Anzahl Proben schnell und kostengünstig an intakten Achänen zu untersuchen. Anhand einer externen Validation kann festgestellt werden, ob sich die Kalibrationen der vorliegenden Arbeit für den zu untersuchenden Probensatz eignen. Gegebenenfalls sollte versucht werden, die Kalibrationen durch weitere Proben zu verbessern.

Die Ramschparzellen einzelner Kreuzungen sollten isoliert voneinander angelegt werden, da eine relativ hohe Fremdbefruchtungsrate ermittelt wurde. Ab der Prüfung von Nachkommen einzelner Pflanzen sollte geselbstetes Saatgut verwendet werden, um später homozygote Linien zu erhalten. Eine weitere Möglichkeit um homozygote Linien zu erhalten wäre die Verwendung von Doppelhaploiden.

Es wäre sinnvoll die Fremdbefruchtungsrate längerfristig an mehreren Orten zu untersuchen, um den Einfluss von Umweltfaktoren einschätzen zu können.

Interessant wäre es auch, andere Züchtungsmethoden bei Saflor zu untersuchen. Eine mögliche Alternative zu den hier untersuchten Methoden könnten beispielsweise synthetische Sorten sein, bei denen Heterosis gezielt genutzt werden kann. 


\section{Zusammenfassung}

Saflor (Carthamus tinctorius L.), eine der ältesten Kulturpflanzen der Welt, stammt vermutlich aus dem vorderen Orient. Es handelt sich um eine einjährige, $30-150 \mathrm{~cm}$ große Pflanze mit gelben, orangenen oder roten Blüten, die besonders für trockene Standorte gut geeignet ist.

Die Färberdistel, wie Saflor auch genannt wird, wurde ursprünglich zum Färben von Stoffen und Lebensmitteln angebaut. Heute dient sie größtenteils der Ölgewinnung. Das Öl enthält bis zu 89\% der ungesättigten Fettsäure Linolsäure und wird meist als Speiseöl verwendet. Der Ölgehalt variiert zwischen $20 \%$ und $45 \%$. Die Hauptanbaugebiete sind Indien, Mexiko, USA, Argentinien, Australien und Äthiopien.

Auf nur etwa $2 \%$ der ökologisch bewirtschafteten Fläche werden in Deutschland Ölpflanzen kultiviert, obwohl eine starke Nachfrage nach ökologisch erzeugtem Pflanzenöl existiert. Die Einführung von Saflor als Ölpflanze für den ökologischen Landbau könnte das Kulturpflanzenspektrum und die Fruchtfolge erweitern. Der Anbau von Saflor ist auch in Deutschland in sommerwarmen und -trockenen Regionen durchaus möglich. $\mathrm{Da}$ in Deutschland bisher jedoch fast keine Saflorzüchtung durchgeführt wurde, mangelt es an Genotypen, die an das Klima angepasst sind. Besonders der niedrige Ölgehalt und die hohe Krankheitsanfälligkeit sind problematisch.

Hauptziel dieser Arbeit war es, methodische Grundlagen der Saflorzüchtung zu untersuchen und ein Konzept für die Züchtung vorzuschlagen. Hierfür sollten drei Züchtungsmethoden (Stammbaumethode, die Methode der natürlichen Selektion und die Einkornramschmethode) untersucht und bewertet werden. Die wichtigsten Zuchtziele waren ein höherer Ölgehalt und ein niedrigerer Krankheitsbefall. $\mathrm{Da}$ die Fremdbefruchtungsrate bei Saflor laut Literaturangaben zwischen unter $10 \%$ und je nach Umweltbedingungen bis zu $50 \%$ variiert, wurde die Fremdbefruchtung ebenfalls untersucht. Gleichzeitig sollte für die Züchtung eine Methode entwickelt werden, mit der Qualitätsmerkmale (Ölgehalt, Linolsäure- und Ölsäuregehalt und Schalenanteil) bei einer großen Anzahl Proben schnell, kostengünstig und möglichst zerstörungsfrei analysiert werden können. 
Für die Qualitätsuntersuchungen wurde die Nah-Infrarot-Reflexions Spektroskopie (NIRS) gewählt. Es wurden Kalibrationen für die Merkmale Ölgehalt, Linolsäure- und Ölsäuregehalt und Schalenanteil an Mehl und intakten Achänen entwickelt. In die Kalibrationen flossen Proben aus den Jahren 2002 bis 2006 von vier verschiedenen Standorten ein. Die vier Standorte waren: Göttingen (südliches Niedersachsen), Hohenheim (BadenWürttemberg), Wilmersdorf (Brandenburg) und Darzau (nordöstliches Niedersachsen). Das wichtigste Merkmal, der Ölgehalt, konnte mittels NIRS an intakten Achänen geschätzt werden. Die Werte der Referenzmethode (Soxhlet) zeigten eine enge Beziehung zu den NIRS-Werten (Bestimmtheitsmaß der Kalibration: $\left.R^{2}=0,87\right)$. Die Bestimmung des Ölgehaltes an Mehl lieferte noch exaktere Ergebnisse $\left(R^{2}=0,91\right)$. Die Messung intakter Achänen bietet jedoch den Vorteil, dass das Saatgut weiterverwendet werden kann. Die Kalibrationen für den Linolsäure- und Ölsäuregehalt (Referenzmethode: Gaschromatographie) und den Schalenanteil waren weniger genau. Hier kann NIRS dazu dienen, eine Vorauswahl aus einer großen Anzahl Proben zu treffen, die anschließend gaschromatographisch untersucht werden. Der Schalenanteil war mit dem Ölgehalt eng negativ korreliert $\left(-0,83^{* *}\right)$. Da eine schnelle Bestimmung des Ölgehaltes möglich ist, verliert die Bestimmung des Schalenanteils an Wichtigkeit.

Die drei verschiedenen Züchtungsmethoden wurden von 2004 bis 2006 anhand von drei Kreuzungen, an denen vier Eltern beteiligt waren, untersucht. Im Vorfeld dieser Arbeit wurden im Jahr 2002 an das europäische Klima angepasste Genotypen mit kanadischen Genotypen mit hohem Ölgehalt gekreuzt. Die Grundlage für die Versuche dieser Arbeit war das Erntegut von jeweils 50 F2-Pflanzen pro Kreuzung. In der Stammbaummethode wurde in den ersten beiden Jahren in Göttingen auf niedrigen Krankheitsbefall (Köpfchenfäule und Alternaria-Blattflecken) und Ölgehalt selektiert.

Für die Methode der natürlichen Selektion wurde für jede Kreuzung eine Mischung des gleichen Ausgangssaatgutes, das in der Stammbaummethode verwendet wurde, hergestellt und dann zwei Jahre unter natürlicher Selektion an drei unterschiedlichen Standorten (Göttingen, Hohenheim, Wilmersdorf) angebaut.

In der Einkornramschmethode wurde ein Nachkomme jeder F2-Pflanze bis zur F6 im Gewächshaus ohne Selektion weitergeführt. 
In 2006 wurden die besten F5-Linien der Stammbaummethode und die F5Parzellen nach natürlicher Selektion an vier Standorten (zusätzlich Darzau) geprüft. Durch den Vergleich mit einer unselektierten Kontrolle pro Kreuzung, die aus einer Mischung von Nachkommen der F6-Pflanzen aus der Einkornramschmethode bestand, wurde der Selektionserfolg beurteilt.

Die Selektion in der Stammbaummethode führte in allen Kreuzungen zu Linien mit geringerer Anfälligkeit für Köpfchenfäule und höherem Ölgehalt im Vergleich zur Kontrolle. In zwei der drei Kreuzungen waren Linien zu finden, die weniger Köpfchenfäule und einen höheren Ölgehalt aufwiesen als der bessere Elter der Kreuzung. Es gab Linien, die einen höheren Einzelpflanzenertrag als die Kontrolle und als der bessere Elter aufwiesen.

Mit geringerem Arbeitsaufwand führte die natürliche Selektion ebenfalls zu guten Erfolgen. Die Anfälligkeit für Köpfchenfäule sank, und der Einzelpflanzenertrag stieg in allen drei Kreuzungen. Der Ölgehalt wurde von der natürlichen Selektion nicht beeinflusst. Für die Züchtung von Saflor wäre daher vorzuschlagen, zunächst die F2- und F3-Generationen der natürlichen Selektion auszusetzen. Anschließend sollten die Nachkommen von Einzelpflanzen geprüft und selektiert werden. Neben der geringeren Krankheitsanfälligkeit sollte der Ölgehalt im Vordergrund stehen, da durch die natürliche Selektion keine Steigerung des Ölgehaltes erreicht wurde.

Versuche zur Fremdbefruchtung innerhalb einer Parzelle wurden 2004 und 2005 in Göttingen durchgeführt. Die Fremdbefruchtung zwischen Parzellen wurden 2004 untersucht. Als Marker für die Fremdbefruchtung wurde die Bestachelung gewählt. Die Fremdbefruchtung zwischen Parzellen lag 2004 in der Mitte der Parzelle bei 6,5\%. Wesentlich höher war sie zwischen Pflanzen innerhalb einer Parzelle. Hier wurden starke Unterschiede zwischen den Jahren festgestellt (2004: 63,1\%; 2005: 29,9\%). Bei der Entwicklung von Linien müssen daher Maßnahmen, wie Isolation oder Selbstungen, gegen die Auskreuzung getroffen werden. 


\section{Literaturverzeichnis}

Baydar, H. and O.Y. Gökmen (2003): Hybrid seed production in safflower (Carthamus tinctorius) following the induction of male sterility by gibberellic acid. Plant Breeding 122: 459-461

Baye, T. and H.C. Becker (2004): Analysing seed weight, fatty acid, oil and protein contents in Vernonia galamensis germplasm by near infrared reflectance spectroscopy. Journal of the American Oil Chemists' Society 81: 651-645

Becker, H. (1993): Pflanzenzüchtung. Ulmer Verlag, Stuttgart

Becker, H.C., C. Damgaard and B. Karlsson (1992): Environmental variation for outcrossing rate in rapeseed. Theoretical and Applied Genetics 84: 303306

Bergmann, J.W., D.E. Baldridge, P.L. Brown, A.L. Dubbs, G.D. Kushnak and N.R. Riveland (1987): Registration of 'Hartmann' safflower. Crop Science 27:1090-1091

Bergmann, J.W., G. Carlson, G. Kushnak, N.R. Riveland and G. Stallknecht (1985): Registration of 'Oker' safflower. Crop Science 25: 1127-1128

Bergmann, J.W., G. Carlson, G. Kushnak, N.R. Riveland, G. Stallknecht, L.E. Welty and D. Wichman (1989): Registration of 'Girard' safflower. Crop Science 29: 828-829

Biertümpfel, A., T. Graf und G. Wurl (2006): Steigerung von Ertragsstabilität und Ölgehalt bei Saflor (Carthamus tinctorius). UFOP-Schrifen 29: 203207

Carvalho, I.S., I. Miranda and H. Pereira (2006): Evaluation of oil composition of some crops suitable for human nutrition. Industrial Crops and Products 24: 75-78

Dajue, L. and H.H. Mündel (1996): Safflower, Carthamus tinctorius L. Promoting the conservation and use of underutilized and neglected crops. 7. International Plant Genetic Resources Institute (IPGRI), Rom, und Institut für Pflanzengenetik und Kulturpflanzenforschung (IPK), Gatersleben.

David, J.L., M. Zivy, M.L. Cardin and P. Brabant (1997): Protein evolution in dynamically managed populations of wheat: adaptive responses to macro-environmental conditions. Theoretical and Applied Genetics 95: 932-941

Deokar, C.C., S.P. Mehtre, D.P. Deshmukh and V.B. Akashe (1999): Alternaria leaf spot resistance in exotic and indigenous safflower germplasm. Sesame and Safflower Newsletter 14: 114-116

Empig, L.T. and W.R. Fehr (1971): Evaluation of methods for generation advance in bulk hybrid soybean populations. Crop Science 11: 51-54 
Engqvist, G.M. and H.C. Becker (1991): Heterosis and epistasis in rapeseed estimated from generation means. Euphytica 58: 31-35

Fassio, A. and D. Cozzolino (2004): Non-destructive prediction of chemical composition in sunflower seeds by near infrared spectroscopy. Industrial Crops and Products 20: 321-329

Fernández-Martínez, J., J. Dominguez Gimenez, A. Jimenez and L. Hernandez (1986): Use of the single seed descent method in breeding safflower (Carthamus tinctorius L.). Plant Breeding 97: 364-367

Fernández-Martínez, J., M. del Rio and A. de Haro (1993): Survey of safflower (Carthamus tinctorius L.) germplasm for variants in fatty acid composition and other seed characters. Euphytica 69: 115-122

Font, R., M. del Río-Celestino and A. de Haro-Bailón (2006): The use of nearinfrared spectroscopy (NIRS) in the study of seed quality components in plant breeding progams. Industrial Crops and Products 24: 307-313

Fontaine, F., J. Hörr and B. Schirmer (2001): Near-infrared reflectance spectroscopy enabels the fast and accurate prediction of the essential amino acid contents in soy, rapeseed meal, sunflower meal, peas, fishmeal, meat meal products, and poultry meal. Journal of Agricultural and Food Chemistry 49: 57-66

Frick, C. und T. Hebeisen (2005): Distelöl aus schweizer Saflor? Agrarforschung 12: 146-151

Geisler, G. (1991): Farbatlas landwirtschaftlicher Kulturpflanzen. Ulmer Verlag, Stuttgart

Haddad, N.I. and F.J. Muehlbauer (1981): Comparison of random bulk population and single-seed-descent methods for lentil breeding. Euphytica 30: 643-651

Hom, N.H. (2004): Pollen genotype effects on seed quality and selection of single seeds by near-infrared reflectance spectroscopy (NIRS) in winter oilseed rape. Klartext, Göttingen

Hom, N.H., H.C. Becker and C. Möllers (2007): Non-destructive analysis of rapeseed quality by NIRS of small seed samples and single seeds. Euphytica 153: 27-34

Horneburg B. (2003): Standortspezifische Sortenentwicklung. Eine Studie mit Landsorten der Linse. Schriften $\mathrm{zu}$ genetischen Ressourcen, Zentralstelle für Agrardokumentation und -information (ZADI). Band 21

http://www.fao.org: Food and Agriculture Organization of the United Nations http://www.vupt.cz: Research Institute for Fodder Crops, Troubsko

Jana, S. and B.S. Khangura (1986): Conservation of diversity in bulk populations of barley (Hordeum vulgare L.). Euphytica 35: 761-776 
Johnson, R.C., J.W. Bergmann and C.R. Flynn (1999): Oil and meal characteristics of core and non-core safflower accessions from USDA collection. Genetic Resouces and Crop Evolution 46: 611-618

Kaffka, S.R. and T.E. Kearney (1998): Safflower production in California, UC Agriculture \& Natural Resources Publication 21565 (http://agric.ucdavis.edu/crops/oilseed/safflower.htm)

Klisiewicz, J.M. and A.L. Urie (1982): Registration of fusarium resistant safflower germplasm. Crop Science 22: 165

Knowles, P.F. (1969): Modification of quantity and quality of safflower oil through plant breeding. Journal of the American Oil Chemists' Society 46: $130-132$

Knowles, P.F. (1976): Safflower. In: N.W. Simmons (Ed.), Evolution in Crop Plants: $31-33$

Knowles, P.F. (1989): Safflower. In: G. Röbbelen, R.K. Downey, A. Ashri (Ed.): Oil Crops of the World, their Breeding and Utilization. McGraw Hill, Inc., New York: 363-374

Lennerts, L. (1984): Ölschrote, Ölkuchen, pflanzliche Öle und Fette. Herkunft, Gewinnung und Verwendung. Verlag A. Strothe, Hannover

Letchworth, M.B. and R.J. Lambert (1998): Pollen parent effects on oil, protein, and starch concentration in maize kernels. Crop Science 38: 363-367

Link, W. (1990): Autofertility and rate of cross-fertilization: crucial characters for breeding synthetic varieties in faba beans (Vicia faba L.). Theoretical and Applied Genetics 79: 713-717

Míka, V., P. Tillmann, R. Koprna, P. Nerušil and V. Kučera (2003): Fast prediction of quality parameters in whole seeds of oilseed rape (Brassica napus L.). Plant, Soil and Environment 49: 141-145

Möllers, C. and A. Schierholt (2002): Genetic variation of palmitate and oil content in a winter oilseed rape doubled haploid population segregating for oleate content. Crop Science 42: 379-384

Moschner, C.R. and B. Biskupek-Korell (2006): Estimating the content of free fatty acids in high-oleic sunflower seeds by near-infrared spectroscopy. European Journal of Lipid Science and Technology 108: 606-613

Moschner, C.R., G. Rühl and B. Biskupek-Korell (2004): Estimating the quality of ground and intact sunflower seeds by near-infrared spectroscopy (NIRS). New directions for a diverse planet: Proceedings of the $4^{\text {th }}$ International Crop Science Congress, Brisbane, Australia 26. Sep.-1. Oct. 2004. ISBN 1-920-842-209

Mündel, H.H. (1996): AC Sunset safflower. Canadian Journal of Plant Science 76: $469-472$ 
Mündel, H.H. and G.B. Schaalje (1988): Use of near infrared reflectance spectroscopy to screen soybean lines for plant nitrogen. Crop Science 28: $157-162$

Mündel, H.H. and H.-C. Huang (2003): Control of major diseases of safflower by breeding for resistance and using cultural practices. Advances in Plant Disease Management: 293-310

Mündel, H.H. and J.P. Braun (1999): Registration of two early-maturing safflower germplasm lines with high oleic acid and high oil content. Crop Science 39: 298-299

Mündel, H.H., H.C. Huang , I.D. Burch and F. Kiehn (1985a): Saffire safflower. Canadian Journal of Plant Science 65: 1079-1081

Mündel, H.H., H.C. Huang and G. Kozub (1985b): Scerotinia head rot in safflower: assessment of resistance and effects on yield and oil content. Canadian Journal of Plant Science 65: 259-265

Mündel, H.H., N.C. Huang, G.C. Kozub and C.R.G. Daniels (1997): Effect of soil moisture, soil temperature and seed-borne Alternaria carthami, on emergence of safflower (Carthamus tinctorius L.). Botanical Bulletin of Academia Sinica 38: 257-262

Mündel, H.H., R. E. Blackshaw, J.R. Byers, H.C. Huang, D.L. Johnson, R. Keon, J. Kubik, R. McKenzie, B. Otto, B. Roth and K. Stanfort (2004): Safflower production on the canadian prairies: revisited in 2004. Agriculture and Agri-Food Canada, Lethbridge Research Centre: 23. ISBN: 0-662-38209-9

Pahlavani, M.H., A.F. Mirlohi and G. Saeidi (2004): Inheritance of flower colour and spininess in safflower (Carthamus tinctorius L.). Journal of Heredity 95: 265-267

Paillard, S., I. Goldringer, J. Enjalbert, M. Trottet, J. David, C. de VallavieillePope and P. Brabant (2000a): Evolution of resistance against powdery mildew in winter wheat populationes conducted under dynamic management. I Is specific seedling resistance selected? Theoretical and Applied Genetics 101: 449-456

Paillard, S., I. Goldringer, J. Enjalbert, M. Trottet, J. David, C. de VallavieillePope and P. Brabant (2000b): Evolution of resistance against powdery mildew in winter wheat populationes conducted under dynamic management. II Adult plant resistance. Theoretical and Applied Genetics 101: $457-462$

Pathirana, R. (1995): Comparison of selection procedures in breeding for seed yield in segregating sesame populations. Euphytica 82: 73-78

Pazdernik, D.L., A.S. Kilam and J.H. Orf (1997): Analysis of amino acid and fatty acid composition in soybean seed, using near infrared reflectance spectroscopy, Agronomy Journal 89: 679-685 
Pérez-Vich, B., L. Velasco and J.M. Fernández-Martínez (1998): Determination of seed oil content and fatty acid composition in sunflower through the analysis of intact seeds, husked seeds, meal and oil by near-infrared reflectance spectroscopy. Journal of the American Oil Chemists' Society 75: $547-555$

Ramachandram, M. and J.V. Goud (1981): Genetic analysis of seed yield, oil content and their components in safflower (Carthamus tinctorius L.). Theoretical and Applied Genetics 60: 191-195

Reinbrecht, C., S. Barth, S. von Witzke-Ehbrecht, M.A. Khan, H.C. Becker, G. Kahnt und W. Claupein (2003): Selektion anbauwürdiger Saflor-Formen für den Ökologischen Landbau aus einem zweijährigen SreeningExperiment. Mitteilung der Gesellschaft für Pflanzenbauwissenschaften 15: $23-26$

Reinbrecht, C., S. Barth, S. von Witzke-Ehbrecht, M.A. Khan, H.C. Becker, G. Kahnt, W. Claupein (2004): Evaluierung von Saflor-Akzessionen für den Ökologischen Landbau. Vorträge für Pflanzenzüchtung 62: 101-103

Salas, G. and W. Friedt (1995): Comparison of pedigree selection and single seed descent for oil yield in linseed (Linum usitatissimum L.). Euphytica 83: $25-32$

Sastry, R.K. and C. Chattopadhyay (2003): Development of Fusarium wiltresistance genotypes in safflower (Carthamus tinctorius L.). European Journal of Plant Pathology 109: 147-151

Scheibe, A. (1938): Zuchtprobleme bei der Sonnenblume. Theoretical and Applied Genetics 10: 126-132

Scheibe, A. (1939): Zucht- und Anbauerfahrungen mit Saflor (Carthamus tinctorius L.). Pflanzenbau 15: 129-159

Scheibe, A. und E. Yekta (1934): Der Saflor, Carthamus tinctorius L., als Ölpflanze. Pflanzenbau 11: 49-67

Shenk, J.S. and M.O. Westerhaus (1991): Population definition, sample selection, and calibration procedures for near infrared reflectance spectroscopy. Crop Science 31: 469-474

SÖL (2003): SÖL-Jahrbuch 2003, Stiftung Ökologie und Landbau

Spaar, D., H. Kleinhempel und R. Fritsche (1990): Diagnose von Krankheiten und Beschädigungen an Kulturpflanzen. Öl und Faserpflanzen. SpringerVerlag: 166

Stuth, J., A. Jama and D. Tolleson (2003): Direct and indirect means of predicting forage quality through near infrared reflectance spectroscopy. Field Crops Research 84: 45-56

Tee, T.S. and C.O. Qualset (1975): Bulk polulations in wheat breeding: comparison of single-seed descent and random bulk methods. Euphytica 24: 393-405 
Thies, W. (1971): Schnelle und einfache Analysen der Fettsäurezusammensetzung in einzelnen Raps-Kotyledonen I. Gaschromatographische und papierchromatographische Methoden. Zeitschrift für Pflanzenzüchtung 65: 181-202

Utz, H.F. (2001): PLABSTAT Version 2N(L). Ein Computerprogramm zur statistischen Analyse von pflanzenzüchterischen Experimenten. Institut für Pflanzenzüchtung, Saatgutforschung und Populationsgenetik der Universität Hohenheim

Utz, H.F. (2005): PLABSTAT Version 3Awin-mini. Ein Computerprogramm zur statistischen Analyse von pflanzenzüchterischen Experimenten. Institut für Pflanzenzüchtung, Saatgutforschung und Populationsgenetik der Universität Hohenheim

Velasco, L., A. Schierholt and H.C. Becker (1998): Performance of nearinfrared reflectance spectroscopy (NIRS) in routine analysis of $\mathrm{C} 18$ unsaturated fatty acids in intact rapeseed. Fett/Lipid 100: 44-48

Velasco, L., B. Pérez-Vich and J.M. Fernández-Martínez (1999b): Nondestructive screening for oleic and linoleic acid in single sunflower achenes by near-infrared reflectance spectroscopy. Crop Science 39: 219-222

Velasco, L., C. Möllers and H.C. Becker (1999a): Estimation of seed weight, oil content and fatty acid composition in intact single seeds of rapeseed (Brassica napus L.) by near-infrared reflectance spectroscopy. Euphytica 106: $79-85$

Weiss, E.A. (1971): Castor, Sesam and Safflower. Barnes and Noble, Inc., New York: 529-744

Weiss, E.A. (2000): Oilseed crops. Chapter: Safflower. $2^{\text {nd }}$ edition. Blackwell Science Ltd. Victoria, Australia: 93-129 


\section{Anhang}

Tab. A I: Termine der Bonituraufnahme

\begin{tabular}{|c|c|c|c|c|}
\hline & Lager & Köpfchenfäule & Alternaria & Fusarium (\%) \\
\hline \multicolumn{5}{|l|}{2004} \\
\hline Göttingen & 09.08. & 10.08. & 10.08. & \\
\hline \multicolumn{5}{|l|}{2005} \\
\hline Göttingen & & 24.08. & 24.08. & \\
\hline \multicolumn{5}{|l|}{2006} \\
\hline $\begin{array}{l}\text { Göttingen } \\
\text { Hohenheim } \\
\text { Wilmersdorf } \\
\text { Darzau }\end{array}$ & & $\begin{array}{l}04.09 . \\
24.08 . \\
10.08 . \\
16.08\end{array}$ & $\begin{array}{l}11.08 . \\
17.08 .\end{array}$ & $\begin{array}{c}22.06 .-26.06 \\
27.06 .-05.07 \\
26.06 .-17.07 \\
27.06\end{array}$ \\
\hline
\end{tabular}

Tab. A II: Ergebnisse der Varianzanalyse zwischen F2:4-Familien

\begin{tabular}{|c|c|c|c|c|c|c|c|c|c|}
\hline \multirow[b]{2}{*}{ Merkmal } & \multicolumn{3}{|c|}{ Kreuzung 1} & \multicolumn{3}{|c|}{ Kreuzung 2} & \multicolumn{3}{|c|}{ Kreuzung 3} \\
\hline & FG & MQ & F-Wert & FG & MQ & F-Wert & FG & MQ & F-Wert \\
\hline Köpfchenfäule & 13 & 1,95 & 10 & 1. & 3,19 & 24 & 13 & 5,07 & $2,16^{*}$ \\
\hline Altern & 13 & 2,35 & 3 & 13 & 2, & 3 & 13 & 1,00 & $3,19^{* *}$ \\
\hline Ölgehalt (\%) & 13 & 59,54 & $5,63^{\star *}$ & 13 & 44,03 & $6,34^{* *}$ & 13 & 27,31 & $2,01^{*}$ \\
\hline
\end{tabular}

Tab. A III: Stammbaummethode 2004 und 2005: phänologische Daten (numerischer Tag) und Pflanzenlänge $(\mathrm{cm})$ in Göttingen, Mittel der Linien und Werte der Eltern

\begin{tabular}{lccccc}
\hline & Blühbeginn & Blühende & Blühdauer & Reife & Pflanzenlänge \\
\hline 2004 & & & & & \\
Kreuzung 1 & 213,16 & 236,46 & 23,30 & 251,48 & 121,94 \\
Kreuzung 2 & 213,02 & 235,12 & 22,10 & 251,52 & 121,30 \\
Kreuzung 3 & 214,76 & 235,24 & 20,48 & 251,28 & 125,48 \\
AC Sunset & 211,67 & 234,67 & 23,00 & 250,67 & 98,67 \\
CR1 & 213,00 & 236,50 & 23,50 & 251,00 & 116,50 \\
Sabina & 213,00 & 236,00 & 23,00 & 252,00 & 133,00 \\
Saffire & 213,00 & 234,00 & 21,00 & 250,00 & 90,00 \\
2005 & & & & & \\
Kreuzung 1 & 206,98 & 230,90 & 23,92 & 251,02 & 93,84 \\
Kreuzung 2 & 206,82 & 231,02 & 24,20 & 251,30 & 91,70 \\
Kreuzung 3 & 209,86 & 233,19 & 23,33 & 251,53 & 87,67 \\
AC Sunset & 209,00 & 231,67 & 22,67 & 249,00 & 70,00 \\
CR1 & 212,00 & 234,00 & 22,00 & 250,00 & 90,00 \\
Sabina & 212,00 & 239,00 & 27,00 & 253,00 & 100,00 \\
Saffire & 209,33 & 232,33 & 23,00 & 249,00 & 80,00 \\
\hline
\end{tabular}


Tab. A IV: Stammbaummethode 2004: Boniturnoten und Ölgehalt (\%) der Eltern

\begin{tabular}{lcccc} 
Elter & Lager & Köpfchenfäule & Alternaria & Ölgehalt (\%) \\
\hline AC Sunset & 1 & 4 & 4 & 21,21 \\
CR1 & 7 & 1 & 1 & 17,83 \\
Sabina & 7 & 1 & 2 & 19,01 \\
Saffire & 3 & 3 & 4 & 23,24 \\
\hline
\end{tabular}


Tab. A V: Stammbaummethode 2004: Selektion der F3-Linien der Kreuzung 1

\begin{tabular}{|c|c|c|c|c|}
\hline Prüfgliednr. & Lager & Köpfchenfäule & Alternaria & Ölgehalt (\%) \\
\hline$\overline{1}$ & 6 & 1 & 2 & \\
\hline 2 & 5 & 4 & 2 & \\
\hline 3 & 3 & 1 & 1 & 18,99 \\
\hline 4 & 3 & 1 & 2 & 18,15 \\
\hline 5 & 5 & 1 & 1 & \\
\hline 6 & 2 & 3 & 2 & \\
\hline 7 & 3 & 1 & 1 & 18,85 \\
\hline 8 & 5 & 1 & 2 & \\
\hline 9 & 3 & 1 & 2 & 18,11 \\
\hline 10 & 2 & 1 & 2 & 21,32 \\
\hline 11 & 5 & 1 & 1 & \\
\hline 12 & 3 & 3 & 1 & \\
\hline 13 & 2 & 1 & 1 & 20,52 \\
\hline 14 & 5 & 1 & 1 & \\
\hline 15 & 2 & 1 & 1 & 24,19 \\
\hline 16 & 2 & 1 & 2 & 16,57 \\
\hline 17 & 3 & 1 & 1 & 18,22 \\
\hline 18 & 3 & 1 & 2 & 19,13 \\
\hline 19 & 2 & 1 & 1 & 20,60 \\
\hline 20 & 2 & 3 & 1 & \\
\hline 21 & 4 & 1 & 1 & 20,54 \\
\hline 22 & 3 & 1 & 1 & 17,64 \\
\hline 23 & 3 & 4 & 1 & \\
\hline 24 & 2 & 1 & 2 & 18,24 \\
\hline 25 & 3 & 1 & 1 & 21,55 \\
\hline 26 & 6 & 1 & 1 & \\
\hline 27 & 4 & 2 & 1 & 14,90 \\
\hline 28 & 2 & 1 & 1 & 22,21 \\
\hline 29 & 2 & 1 & 1 & 18,58 \\
\hline 30 & 2 & 1 & 1 & 21,68 \\
\hline 31 & 4 & 1 & 4 & \\
\hline 32 & 2 & 1 & 1 & 19,59 \\
\hline 33 & 4 & 1 & 1 & 19,92 \\
\hline 34 & 3 & 2 & 1 & 18,53 \\
\hline 35 & 3 & 1 & 1 & 18,72 \\
\hline 36 & 4 & 1 & 2 & 22,75 \\
\hline 37 & 2 & 2 & 1 & 21,23 \\
\hline 38 & 2 & 1 & 1 & 22,94 \\
\hline 39 & 3 & 1 & 3 & 21,78 \\
\hline 40 & 3 & 1 & 1 & 17,57 \\
\hline 41 & 2 & 5 & 4 & \\
\hline 42 & 2 & 1 & 1 & 17,73 \\
\hline 43 & 2 & 1 & 3 & 18,26 \\
\hline 44 & 2 & 1 & 2 & 18,24 \\
\hline 45 & 3 & 1 & 1 & 20,46 \\
\hline 46 & 3 & 1 & 1 & 21,33 \\
\hline 47 & 3 & 1 & 1 & 18,83 \\
\hline 48 & 3 & 1 & 2 & 17,80 \\
\hline 49 & 3 & 3 & 1 & \\
\hline 50 & 7 & 1 & 3 & \\
\hline Mittel Linien & 3,1 & 1,5 & 1,5 & 19,59 \\
\hline
\end{tabular}

grau: positiv selektierte Linien, fett: im betreffenden Merkmal unter Selektionsschwelle 
Tab. A VI: Stammbaummethode 2004: Selektion der F3-Linien der Kreuzung 2

\begin{tabular}{|c|c|c|c|c|}
\hline Prüfgliednr. & Lager & Köpfchenfäule & Alternaria & Ölgehalt (\%) \\
\hline$\overline{51}$ & 3 & 1 & 1 & 20,44 \\
\hline 52 & 3 & 4 & 1 & \\
\hline 53 & 3 & 1 & 3 & 21,87 \\
\hline 54 & 2 & 1 & 3 & 18,66 \\
\hline 55 & 4 & 1 & 2 & 26,41 \\
\hline 56 & 3 & 1 & 2 & 20,07 \\
\hline 57 & 4 & 1 & 1 & 15,84 \\
\hline 58 & 2 & 1 & 2 & 21,34 \\
\hline 59 & 5 & 1 & 6 & \\
\hline 60 & 4 & 3 & 2 & \\
\hline 61 & 5 & 3 & 2 & \\
\hline 62 & 2 & 3 & 2 & \\
\hline 63 & 4 & 1 & 2 & 20,40 \\
\hline 64 & 6 & 1 & 1 & \\
\hline 65 & 4 & 1 & 3 & 18,05 \\
\hline 66 & 2 & 1 & 1 & 17,25 \\
\hline 67 & 2 & 1 & 3 & 21,81 \\
\hline 68 & 4 & 1 & 3 & 18,71 \\
\hline 69 & 3 & 1 & 4 & 21,32 \\
\hline 70 & 6 & 1 & 1 & \\
\hline 71 & 5 & 2 & 3 & 22,17 \\
\hline 72 & 6 & 1 & 4 & \\
\hline 73 & 5 & 1 & 3 & 22,60 \\
\hline 74 & 4 & 1 & 5 & 24,82 \\
\hline 75 & 2 & 1 & 3 & 23,17 \\
\hline 76 & 3 & 1 & 1 & 20,75 \\
\hline 77 & 5 & 1 & 2 & 23,62 \\
\hline 78 & 7 & 1 & 4 & \\
\hline 79 & 5 & 2 & 1 & 17,56 \\
\hline 80 & 5 & 1 & 4 & 22,08 \\
\hline 81 & 3 & 1 & 3 & 21,75 \\
\hline 82 & 4 & 1 & 1 & 21,03 \\
\hline 83 & 3 & 1 & 3 & 22,16 \\
\hline 84 & 3 & 1 & 2 & 23,51 \\
\hline 85 & 3 & 1 & 6 & \\
\hline 86 & 3 & 1 & 1 & 20,20 \\
\hline 87 & 3 & 1 & 3 & 20,64 \\
\hline 88 & 2 & 1 & 2 & 21,00 \\
\hline 89 & 5 & 1 & 1 & 18,50 \\
\hline 90 & 3 & 1 & 2 & 22,77 \\
\hline 91 & 7 & 7 & 2 & \\
\hline 92 & 4 & 9 & 1 & \\
\hline 93 & 4 & 1 & 1 & 18,45 \\
\hline 94 & 4 & 1 & 4 & 20,57 \\
\hline 95 & 5 & 5 & 2 & \\
\hline 96 & 7 & 1 & 3 & \\
\hline 97 & 4 & 1 & 1 & 16,71 \\
\hline 98 & 5 & 7 & 5 & \\
\hline 99 & 3 & 7 & 2 & \\
\hline 100 & 5 & 1 & 3 & 22,16 \\
\hline Mittel Linien & 4,0 & 1,8 & 2,5 & 20,83 \\
\hline
\end{tabular}

grau: positiv selektierte Linien, fett: im betreffenden Merkmal unter Selektionsschwelle 
Tab. A VII: Stammbaummethode 2004: Selektion der F3-Linien der Kreuzung 3

\begin{tabular}{|c|c|c|c|c|}
\hline Prüfgliednr. & Lager & Köpfchenfäule & Alternaria & Ölgehalt (\%) \\
\hline$\overline{151}$ & 4 & 1 & 1 & 16,06 \\
\hline 152 & 4 & 1 & 3 & 13,83 \\
\hline 153 & 2 & 1 & 1 & 14,18 \\
\hline 154 & 4 & 1 & 1 & 13,53 \\
\hline 155 & 4 & 1 & 1 & 16,73 \\
\hline 156 & 4 & 1 & 1 & 14,15 \\
\hline 157 & 3 & 1 & 2 & 19,85 \\
\hline 158 & 2 & 1 & 1 & 19,24 \\
\hline 159 & 3 & 1 & 2 & 17,89 \\
\hline 160 & 5 & 2 & 1 & \\
\hline 161 & 3 & 1 & 2 & 11,35 \\
\hline 162 & 4 & 1 & 1 & 9,43 \\
\hline 163 & 4 & 1 & 1 & 11,08 \\
\hline 164 & 3 & 1 & 1 & 18,40 \\
\hline 165 & 3 & 1 & 2 & 17,00 \\
\hline 166 & 2 & 1 & 1 & 17,67 \\
\hline 167 & 3 & 9 & 5 & \\
\hline 168 & 3 & 4 & 2 & \\
\hline 169 & 4 & 1 & 3 & 15,78 \\
\hline 170 & 4 & 1 & 2 & 16,83 \\
\hline 171 & 4 & 1 & 1 & 15,86 \\
\hline 172 & 3 & 1 & 3 & 18,74 \\
\hline 173 & 2 & 2 & 1 & \\
\hline 174 & 2 & 2 & 1 & \\
\hline 175 & 4 & 1 & 1 & 14,70 \\
\hline 176 & 2 & 2 & 2 & \\
\hline 177 & 2 & 1 & 2 & 14,27 \\
\hline 178 & 2 & 1 & 1 & 12,86 \\
\hline 179 & 3 & 1 & 2 & 17,04 \\
\hline 180 & 2 & 1 & 1 & 20,01 \\
\hline 181 & 2 & 1 & 1 & 18,68 \\
\hline 182 & 2 & 1 & 1 & 17,52 \\
\hline 183 & 4 & 1 & 1 & 14,86 \\
\hline 184 & 4 & 1 & 1 & 16,12 \\
\hline 185 & 2 & 1 & 1 & 9,93 \\
\hline 186 & 3 & 1 & 2 & 9,51 \\
\hline 187 & 5 & 1 & 1 & \\
\hline 188 & 3 & 1 & 1 & 16,42 \\
\hline 189 & 3 & 1 & 1 & 17,87 \\
\hline 190 & 4 & 1 & 2 & 14,99 \\
\hline 191 & 3 & 1 & 1 & 17,81 \\
\hline 192 & 3 & 2 & 2 & \\
\hline 193 & 4 & 1 & 1 & 16,41 \\
\hline 194 & 4 & 1 & 2 & 11,92 \\
\hline 195 & 3 & 3 & 2 & \\
\hline 196 & 4 & 1 & 1 & 10,12 \\
\hline 197 & 3 & 1 & 1 & 14,80 \\
\hline 198 & 3 & 1 & 2 & 17,64 \\
\hline 199 & 3 & 5 & 5 & \\
\hline 200 & 4 & 1 & 1 & 12,29 \\
\hline Mittel Linien & 3,2 & 1,4 & 1,6 & 15,34 \\
\hline
\end{tabular}

grau: positiv selektierte Linien, fett: im betreffenden Merkmal unter Selektionsschwelle 
Tab. A VIII: Stammbaummethode 2005: Boniturnoten und Ölgehalt (\%) der Eltern

\begin{tabular}{lccc}
\hline Elter & Köpfchenfäule & Alternaria & Ölgehalt (\%) \\
\hline AC Sunset & 5,3 & 2,0 & 16,46 \\
CR1 & 2,0 & 2,3 & 17,74 \\
Sabina & 2,0 & 2,0 & 22,30 \\
Saffire & 2,5 & 3,5 & 24,49 \\
\hline
\end{tabular}

Tab. A IX: Stammbaummethode 2005: Selektion der F2:4-Familien der Kreuzung 1

\begin{tabular}{lccc}
\hline Prüfgliednr. & Köpfchenfäule & Alternaria & Ölgehalt (\%) \\
\hline 10 & 3,14 & 4,12 & 18,29 \\
13 & 3,29 & 3,14 & 19,92 \\
15 & 2,86 & 2,14 & 23,84 \\
19 & 2,71 & 2,29 & 19,05 \\
21 & 4,17 & 2,83 & 16,17 \\
25 & 4,43 & 3,47 & 16,68 \\
28 & 3,43 & 2,43 & 22,88 \\
30 & 4,00 & 3,01 & 21,31 \\
$(36)$ & 3,60 & 3,00 & 18,13 \\
37 & 3,57 & 2,86 & 19,77 \\
38 & 3,71 & 3,03 & 21,19 \\
39 & 4,00 & 4,00 & 20,65 \\
45 & 3,43 & 3,71 & 19,70 \\
46 & 4,33 & 2,50 & 19,19 \\
\hline Mittel & 3,62 & 3,04 & 19,77 \\
\hline
\end{tabular}

grau: positiv selektierte Familie, dunkelgrau: Wert liegt über dem Mittel, in Klammern: zu wenig Saatgut 
Tab. A X: Stammbaummethode 2005: Selektion der F2:4-Familien der Kreuzung 2

\begin{tabular}{lccc}
\hline Prüfgliednr. & Köpfchenfäule & Alternaria & Ölgehalt (\%) \\
\hline 53 & 2,43 & 3,57 & 21,48 \\
55 & 2,43 & 2,86 & 23,65 \\
67 & 3,00 & 2,57 & 21,47 \\
71 & 4,00 & 4,29 & 19,82 \\
73 & 2,71 & 2,86 & 18,24 \\
74 & 4,14 & 3,29 & 17,62 \\
75 & 4,71 & 2,71 & 16,79 \\
77 & 3,57 & 3,71 & 21,32 \\
80 & 3,43 & 3,43 & 20,25 \\
81 & 3,00 & 4,00 & 18,27 \\
83 & 3,33 & 2,33 & 16,15 \\
84 & 3,00 & 2,71 & 19,95 \\
90 & 3,29 & 2,14 & 20,33 \\
100 & 2,57 & 2,71 & 19,52 \\
\hline Mittel & 3,26 & 3,09 & 19,63 \\
\hline grau: positiv selektierte Familie, dunkelgrau: Wert liegt über dem Mittel
\end{tabular}

Tab. A XI: Stammbaummethode 2005: Selektion der F2:4-Familien der Kreuzung 3

\begin{tabular}{lccc}
\hline Prüfgliednr. & Köpfchenfäule & Alternaria & Ölgehalt (\%) \\
\hline 157 & 5,80 & 3,20 & 15,38 \\
158 & 4,50 & 2,50 & 14,13 \\
159 & 5,67 & 2,67 & 13,74 \\
164 & 3,67 & 3,17 & 16,74 \\
165 & 3,00 & 2,25 & 16,41 \\
166 & 3,00 & 2,75 & 12,06 \\
172 & 3,17 & 2,67 & 17,79 \\
$(179)$ & 3,33 & 3,00 & 16,49 \\
180 & 3,00 & 2,29 & 17,89 \\
181 & 3,67 & 2,17 & 15,77 \\
182 & 2,40 & 2,00 & 16,80 \\
189 & 3,83 & 2,33 & 15,55 \\
191 & 2,50 & 2,00 & 16,90 \\
198 & 4,25 & 2,25 & 12,82 \\
\hline Mittel & 3,70 & 2,52 & 15,61 \\
\hline
\end{tabular}

grau: positiv selektierte Familie, dunkelgrau: Wert liegt über dem Mittel, in Klammern: zu wenig Saatgut 
Tab. A XII: Stammbaummethode 2006: phänologische Daten (numerischer Tag) und Pflanzenlänge $(\mathrm{cm})$ im Mittel über die Standorte

\begin{tabular}{lccccc}
\hline & Blühbeginn & Blühende & Blühdauer & Reife & Pflanzenlänge \\
\hline Kreuzung 1 & & & & & \\
Elter 1 (AC Sunset) & 197,32 & 215,11 & 17,88 & 236,73 & 65,01 \\
Elter 2 (CR1) & 199,82 & 218,40 & 19,11 & 240,18 & 87,67 \\
Mittel der Linien (L) & 199,00 & 218,40 & 19,42 & 239,88 & 80,29 \\
Minimum Linien & 197,56 & 215,56 & 17,82 & 238,86 & 71,05 \\
Maximum Linien & 200,11 & 223,27 & 23,22 & 240,93 & 86,15 \\
Kontrolle (K) & 198,55 & 217,26 & 18,76 & 239,85 & 81,01 \\
L - K & 0,45 & 1,14 & 0,66 & 0,03 & $-0,72$ \\
Kreuzung 2 & & & & & \\
Elter 1 (Saffire) & 198,64 & 217,77 & 19,14 & 239,68 & 76,37 \\
Elter 2 (CR1) & 199,82 & 218,40 & 19,11 & 240,18 & 87,67 \\
Mittel der Linien (L) & 199,27 & 218,48 & 19,19 & 240,09 & 82,15 \\
Minimum Linien & 197,78 & 216,51 & 17,56 & 239,43 & 76,47 \\
Maximum Linien & 200,38 & 221,73 & 21,73 & 240,82 & 88,30 \\
Kontrolle (K) & 198,64 & 217,82 & 19,01 & 240,15 & 86,42 \\
L - K & 0,63 & 0,66 & 0,18 & $-0,06$ & $-4,27$ \\
Kreuzung 3 & & & & & \\
Elter 1 (Sabina) & 202,69 & 222,99 & 20,33 & 246,18 & 110,50 \\
Elter 2 (AC Sunset) & 197,14 & 211,60 & 14,47 & 236,94 & 81,28 \\
Mittel der Linien (L) & 201,35 & 220,41 & 19,02 & 244,96 & 93,56 \\
Minimum Linien & 197,39 & 213,55 & 15,24 & 243,10 & 86,49 \\
Maximum Linien & 205,58 & 227,35 & 23,71 & 246,63 & 103,54 \\
Kontrolle (K) & 200,12 & 218,53 & 18,40 & 243,28 & 102,51 \\
L - K & 1,23 & 1,88 & 0,62 & 1,68 & $-8,95$ \\
\hline
\end{tabular}


Tab. A XIII: Stammbaummethode 2006: Linolsäure- und Ölsäuregehalt (\%) im Mittel über die Standorte

\begin{tabular}{lcc}
\hline & Linolsäure & Ölsäure \\
\hline Kreuzung 1 & & \\
Elter 1 (AC Sunset) & 80,04 & 9,76 \\
Elter 2 (CR1) & 81,67 & 9,41 \\
Mittel der Linien (L) & 82,4 & 8,76 \\
Minimum Linien & 81,56 & 7,74 \\
Maximum Linien & 83,89 & 9,76 \\
Kontrolle (K) & 82,55 & 8,58 \\
L - K & $-0,15$ & 0,18 \\
Kreuzung 2 & & \\
Elter 1 (Saffire) & 82,04 & 8,8 \\
Elter 2 (CR1) & 81,67 & 9,41 \\
Mittel der Linien (L) & 82,59 & 8,73 \\
Minimum Linien & 81,55 & 7,49 \\
Maximum Linien & 84,19 & 9,58 \\
Kontrolle (K) & 82,36 & 9,27 \\
L - K & 0,23 & $-0,54$ \\
Kreuzung 3 & & \\
Elter 1 (Sabina) & 80,16 & 9,07 \\
Elter 2 (AC Sunset) & 77,55 & 11,89 \\
Mittel der Linien (L) & 80,94 & 9,21 \\
Minimum Linien & 78,58 & 7,89 \\
Maximum Linien & 82,61 & 10,73 \\
Kontrolle (K) & 82,08 & 8,66 \\
L - K & $-1,14$ & 0,55 \\
\hline
\end{tabular}


Tab. A XIV: Stammbaummethode 2006: F5-Linien, Eltern und Kontrolle der Kreuzung 1, gemittelt über vier Standorte (Alternaria zwei Standorte)

Prüfglied- Köpfchen- Alternaria Fusarium Ölgehalt EP-Ertrag TKG Schalen-

\begin{tabular}{|c|c|c|c|c|c|c|c|}
\hline nummer & fäule & & $(\%)$ & $(\%)$ & (g) & (g) & anteil (\%) \\
\hline 13,2 & 3,40 & 3,00 & 11,99 & 22,83 & 18,49 & 32,00 & 58,98 \\
\hline 13,4 & 2,80 & 2,50 & 13,83 & 24,13 & 17,54 & 30,40 & 55,53 \\
\hline 13,5 & 3,06 & 3,25 & 7,83 & 24,13 & 14,48 & 32,70 & 56,45 \\
\hline 13,6 & 2,74 & 2,50 & 10,27 & 20,26 & 16,84 & 32,90 & 61,29 \\
\hline 15,2 & 3,03 & 2,75 & 14,02 & 26,24 & 14,69 & 32,40 & 54,73 \\
\hline 15,3 & 2,93 & 3,00 & 22,30 & 22,13 & 15,23 & 35,00 & 61,71 \\
\hline 15,4 & 3,00 & 3,00 & 18,78 & 23,64 & 13,93 & 31,80 & 62,27 \\
\hline 15,6 & 3,01 & 4,00 & 20,57 & 24,72 & 15,51 & 32,20 & 53,40 \\
\hline 15,7 & 2,69 & 2,50 & 19,42 & 24,89 & 16,00 & 33,60 & 55,21 \\
\hline 19,3 & 2,53 & 2,75 & 11,96 & 23,65 & 20,46 & 28,40 & 62,95 \\
\hline 19,4 & 2,13 & 2,50 & 8,75 & 23,98 & 15,48 & 29,60 & 60,88 \\
\hline 19,5 & 2,51 & 2,75 & 8,62 & 23,63 & 19,95 & 29,70 & 62,89 \\
\hline 19,7 & 2,78 & 2,50 & 12,07 & 23,35 & 18,99 & 31,10 & 58,02 \\
\hline 28,1 & 2,27 & 2,75 & 11,10 & 25,28 & 19,59 & 30,20 & 54,62 \\
\hline 28,3 & 2,49 & 2,50 & 12,94 & 25,72 & 19,94 & 28,10 & 55,01 \\
\hline 28,6 & 2,87 & 3,00 & 15,26 & 24,71 & 19,68 & 29,60 & 53,35 \\
\hline 28,7 & 2,73 & 2,75 & 26,20 & 24,31 & 14,60 & 30,70 & 57,76 \\
\hline 30,2 & 2,85 & 2,75 & 17,66 & 24,66 & 20,72 & 33,50 & 55,79 \\
\hline 30,3 & 3,23 & 2,50 & 10,39 & 22,90 & 17,08 & 32,90 & 55,72 \\
\hline 30,4 & 3,04 & 2,50 & 18,75 & 23,46 & 21,03 & 33,20 & 52,53 \\
\hline 30,5 & 3,14 & 2,25 & 17,93 & 23,34 & 20,02 & 33,00 & 56,37 \\
\hline 37,1 & 2,87 & 2,75 & 22,49 & 23,81 & 18,17 & 34,00 & 57,64 \\
\hline 37,3 & 2,89 & 2,50 & 14,43 & 20,19 & 18,08 & 32,30 & 64,41 \\
\hline 37,6 & 2,43 & 3,00 & 19,33 & 22,37 & 16,53 & 32,10 & 59,86 \\
\hline 37,7 & 2,68 & 2,25 & 13,30 & 22,95 & 23,91 & 33,20 & 57,88 \\
\hline 38,1 & 3,07 & 2,75 & 17,03 & 24,65 & 18,39 & 31,80 & 57,78 \\
\hline 38,2 & 2,99 & 4,00 & 9,27 & 24,64 & 18,49 & 32,30 & 55,46 \\
\hline 38,4 & 3,29 & 2,50 & 14,96 & 24,16 & 21,54 & 34,10 & 56,19 \\
\hline 38,7 & 2,76 & 2,75 & 16,26 & 25,06 & 24,57 & 34,80 & 53,89 \\
\hline AC Sunset & 3,86 & 4,50 & 2,43 & 25,13 & 9,92 & 30,33 & 53,20 \\
\hline CR 1 & 2,58 & 1,75 & 18,05 & 22,47 & 19,60 & 35,38 & 59,88 \\
\hline Kontrolle & 3,26 & 2,50 & 4,59 & 22,90 & 19,87 & 33,10 & 57,68 \\
\hline
\end{tabular}

grau: besser als Kontrolle, fett: besser als besserer Elter 
Tab. A XV: Stammbaummethode 2006: F5-Linien, Eltern und Kontrolle der Kreuzung 2, gemittelt über vier Standorte (Alternaria zwei Standorte)

Prüfglied- Köpfchen- Alternaria Fusarium Ölgehalt EP-Ertrag TKG Schalen-

\begin{tabular}{|c|c|c|c|c|c|c|c|}
\hline nummer & fäule & & $(\%)$ & $(\%)$ & (g) & (g) & anteil (\%) \\
\hline 53,1 & 2,38 & 4,00 & 10,01 & 25,08 & 20,95 & 30,50 & 56,17 \\
\hline 53,2 & 2,53 & 4,50 & 19,90 & 23,91 & 12,81 & 32,00 & 54,68 \\
\hline 53,3 & 2,09 & 2,75 & 12,27 & 25,68 & 21,99 & 31,40 & 57,59 \\
\hline 53,5 & 2,78 & 3,75 & 13,97 & 25,31 & 15,47 & 30,40 & 56,92 \\
\hline 55,1 & 2,37 & 3,75 & 21,65 & 26,53 & 17,62 & 28,80 & 53,90 \\
\hline 55,2 & 2,18 & 3,25 & 22,40 & 25,56 & 16,93 & 32,10 & 54,40 \\
\hline 55,3 & 2,22 & 4,00 & 17,36 & 26,89 & 12,73 & 30,90 & 56,06 \\
\hline 55,4 & 2,62 & 3,50 & 15,99 & 24,17 & 21,19 & 32,10 & 54,42 \\
\hline 55,5 & 2,90 & 2,00 & 14,98 & 24,63 & 18,76 & 32,40 & 57,74 \\
\hline 67,1 & 2,43 & 3,00 & 20,53 & 25,99 & 19,52 & 32,20 & 54,51 \\
\hline 67,2 & 2,18 & 4,00 & 17,19 & 24,18 & 14,34 & 33,30 & 59,72 \\
\hline 67,5 & 2,36 & 3,50 & 20,40 & 25,48 & 17,11 & 30,90 & 57,35 \\
\hline 67,6 & 2,91 & 3,00 & 23,84 & 24,59 & 15,94 & 32,90 & 59,88 \\
\hline 73,1 & 2,92 & 3,25 & 20,00 & 25,55 & 20,66 & 35,90 & 54,19 \\
\hline 73,2 & 2,91 & 2,25 & 14,20 & 22,47 & 19,95 & 36,20 & 60,30 \\
\hline 73,3 & 2,21 & 2,75 & 22,32 & 24,09 & 20,34 & 34,10 & 55,29 \\
\hline 73,6 & 2,99 & 3,75 & 14,75 & 23,89 & 19,49 & 35,90 & 58,44 \\
\hline 84,3 & 2,71 & 2,75 & 19,78 & 23,55 & 25,33 & 32,20 & 59,70 \\
\hline 84,5 & 2,67 & 2,75 & 9,59 & 25,42 & 13,23 & 32,10 & 50,91 \\
\hline 84,6 & 2,70 & 2,75 & 19,65 & 24,99 & 17,51 & 31,80 & 54,26 \\
\hline 84,7 & 2,11 & 3,00 & 19,98 & 24,60 & 17,81 & 33,60 & 54,31 \\
\hline 90,1 & 2,49 & 2,75 & 14,85 & 24,55 & 18,28 & 33,60 & 53,92 \\
\hline 90,3 & 3,13 & 3,25 & 24,33 & 25,88 & 21,31 & 35,00 & 54,03 \\
\hline 90,5 & 2,20 & 3,00 & 14,19 & 25,29 & 21,64 & 33,70 & 57,04 \\
\hline 90,6 & 2,43 & 2,50 & 21,58 & 25,18 & 15,48 & 31,50 & 53,08 \\
\hline 100,1 & 2,37 & 2,25 & 17,01 & 22,80 & 18,05 & 33,80 & 57,14 \\
\hline 100,4 & 2,46 & 3,00 & 21,05 & 21,42 & 22,13 & 33,90 & 56,90 \\
\hline 100,6 & 2,35 & 2,75 & 20,05 & 22,59 & 23,00 & 35,90 & 58,99 \\
\hline 100,7 & 2,22 & 2,50 & 15,44 & 23,50 & 20,80 & 33,00 & 59,46 \\
\hline Saffire & 2,84 & 4,50 & 12,34 & 25,74 & 15,97 & 33,80 & 53,50 \\
\hline CR 1 & 2,58 & 1,75 & 18,05 & 22,47 & 19,60 & 35,38 & 59,88 \\
\hline Kontrolle & 2,90 & 2,75 & 6,05 & 23,66 & 17,56 & 31,50 & 53,64 \\
\hline
\end{tabular}

grau: besser als Kontrolle, fett: besser als besserer Elter 
Tab. A XVI: Stammbaummethode 2006: F5-Linien, Eltern und Kontrolle der Kreuzung 3, gemittelt über zwei Standorte

\begin{tabular}{|c|c|c|c|c|c|c|c|}
\hline $\begin{array}{l}\text { Prüfglied- } \\
\text { nummer }\end{array}$ & $\begin{array}{l}\text { Köpfchen- } \\
\text { fäule }\end{array}$ & Alternaria & $\begin{array}{c}\text { Fusarium } \\
(\%)\end{array}$ & $\begin{array}{c}\text { Ölgehalt } \\
(\%)\end{array}$ & $\begin{array}{c}\text { EP-Ertrag } \\
\text { (g) }\end{array}$ & $\begin{array}{l}\text { TKG } \\
\text { (g) }\end{array}$ & $\begin{array}{l}\text { Schalen- } \\
\text { anteil (\%) }\end{array}$ \\
\hline 164,1 & 3,18 & 1,61 & 29,61 & 19,86 & 25,31 & 32,00 & 70,76 \\
\hline 164,3 & 3,61 & 2,41 & 9,48 & 19,44 & 27,56 & 32,10 & 64,50 \\
\hline 164,4 & 3,54 & 2,29 & 19,39 & 20,49 & 23,91 & 34,50 & 63,97 \\
\hline 164,6 & 3,82 & 1,73 & 23,90 & 19,78 & 29,77 & 37,00 & 66,88 \\
\hline 165,1 & 3,73 & 1,37 & 12,17 & 20,03 & 17,40 & 28,40 & 64,92 \\
\hline 165,4 & 3,27 & 2,98 & 13,13 & 21,08 & 19,80 & 29,30 & 62,69 \\
\hline 165,7 & 3,45 & 2,28 & 10,82 & 18,28 & 25,92 & 32,30 & 69,65 \\
\hline 172,1 & 2,74 & 2,34 & 20,43 & 20,64 & 31,32 & 32,70 & 70,11 \\
\hline 172,2 & 4,01 & 1,95 & 11,98 & 19,01 & 25,25 & 35,40 & 70,40 \\
\hline 172,4 & 3,40 & 2,33 & 23,78 & 22,75 & 30,53 & 32,70 & 68,26 \\
\hline 172,6 & 4,00 & 2,95 & 12,69 & 17,00 & 18,88 & 32,00 & 73,18 \\
\hline 180,2 & 3,96 & 3,01 & 19,61 & 21,23 & 26,91 & 33,90 & 65,07 \\
\hline 180,3 & 3,55 & 1,94 & 15,02 & 22,16 & 19,38 & 30,20 & 62,96 \\
\hline 180,4 & 4,77 & 1,97 & 23,53 & 19,02 & 17,66 & 28,80 & 63,67 \\
\hline 180,7 & 4,40 & 2,22 & 18,82 & 20,30 & 25,84 & 34,20 & 68,11 \\
\hline 181,1 & 3,20 & 2,64 & 7,31 & 20,33 & 25,29 & 30,50 & 64,95 \\
\hline 181,3 & 3,92 & 2,72 & 11,65 & 23,56 & 33,35 & 33,00 & 58,21 \\
\hline 181,4 & 2,92 & 2,70 & 23,46 & 21,78 & 36,74 & 31,20 & 60,85 \\
\hline 181,5 & 2,74 & 1,98 & 8,05 & 19,07 & 24,14 & 32,30 & 68,82 \\
\hline 182,1 & 3,62 & 1,69 & 17,77 & 21,68 & 19,95 & 27,80 & 63,16 \\
\hline 182,3 & 3,51 & 1,58 & 6,95 & 17,19 & 13,34 & 24,00 & 70,54 \\
\hline 182,4 & 3,75 & 1,70 & 12,05 & 21,50 & 24,99 & 27,30 & 65,78 \\
\hline 182,6 & 3,21 & 2,05 & 14,47 & 19,77 & 23,35 & 28,30 & 62,37 \\
\hline 191,2 & 3,30 & 3,01 & 7,36 & 19,58 & 16,50 & 29,00 & 65,86 \\
\hline 191,3 & 4,39 & 2,80 & 19,93 & 17,70 & 10,13 & 27,70 & 69,66 \\
\hline 191,6 & 3,23 & 2,38 & 16,88 & 20,36 & 21,47 & 31,20 & 64,72 \\
\hline 191,7 & 3,95 & 1,86 & 21,56 & 22,20 & 27,19 & 32,70 & 63,79 \\
\hline Sabina & 2,04 & 1,37 & 6,08 & 20,95 & 19,91 & 31,15 & 64,44 \\
\hline AC Sunset & 4,81 & 4,66 & 3,67 & 18,47 & 14,50 & 28,45 & 65,13 \\
\hline Kontrolle & 3,67 & 2,14 & 3,36 & 21,75 & 22,24 & 30,90 & 61,34 \\
\hline
\end{tabular}

grau: besser als Kontrolle, fett: besser als besserer Elter 
Tab. A XVII: Ergebnisse der Varianzanalyse für den Ölgehalt (\%)

\begin{tabular}{lcccccc}
\hline Varianzursache & FG & MQ & Var.komp. & F-Wert & GD $(\mathbf{0 , 0 5})$ \\
\hline Standort (O) & 3 & 308,79 & 12,28 & 22,09 & $* *$ & 3,00 \\
Kreuzung (C) & 2 & 55,01 & 1,57 & 11,33 & $* *$ & 1,27 \\
Wiederholungen innerhalb Orte & 4 & 13,98 & 0,76 & 2,88 & & 2,07 \\
CxO & 6 & 23,43 & 2,32 & 4,83 & $*$ & 2,54 \\
Fehler der Großteilstücke & 8 & 4,86 & 0,38 & 1,45 & & 2,62 \\
Herkunftsort (H) & 3 & 6,53 & 0,13 & 1,95 & & 1,07 \\
HxC & 6 & 14,91 & 1,45 & 4,46 & $* *$ & 1,85 \\
HxO & 9 & 8,49 & 0,86 & 2,54 & $*$ & 2,14 \\
HxCxO & 18 & 6,93 & 1,79 & 2,07 & $*$ & 3,71 \\
Fehler der Kleinteilstücke & 36 & 3,34 & 3,34 & & & \\
Total & 95 & & & & & \\
\hline
\end{tabular}


Tab. A XVIII: Fremdbefruchtung zwischen Parzellen 2004

\begin{tabular}{|c|c|c|c|}
\hline & $\begin{array}{c}\text { Anzahl } \\
\text { Nachkommen }\end{array}$ & $\begin{array}{c}\text { bestachelte } \\
\text { Nachkommen }\end{array}$ & $\begin{array}{l}\text { Fremdbefruchtung } \\
(\%)\end{array}$ \\
\hline \multicolumn{4}{|l|}{ Rand 1} \\
\hline & 35 & 2 & 5,71 \\
\hline & 35 & 3 & 8,57 \\
\hline & 32 & 3 & 9,38 \\
\hline & 34 & 2 & 5,88 \\
\hline & 46 & 3 & 6,52 \\
\hline & 24 & 4 & 16,67 \\
\hline & 43 & 3 & 6,98 \\
\hline & 38 & 4 & 10,53 \\
\hline & 31 & 3 & 9,68 \\
\hline & 35 & 6 & 17,14 \\
\hline \multicolumn{4}{|l|}{ Mitte } \\
\hline & 34 & 4 & 11,76 \\
\hline & 44 & 5 & 11,36 \\
\hline & 44 & 0 & 0,00 \\
\hline & 48 & 3 & 6,25 \\
\hline & 34 & 2 & 5,88 \\
\hline & 39 & 3 & 7,69 \\
\hline & 37 & 2 & 5,41 \\
\hline & 30 & 1 & 3,33 \\
\hline & 39 & 4 & 10,26 \\
\hline & 33 & 1 & 3,03 \\
\hline \multicolumn{4}{|l|}{ Rand 2} \\
\hline & 37 & 6 & 16,22 \\
\hline & 20 & 4 & 20,00 \\
\hline & 26 & 3 & 11,54 \\
\hline & 29 & 4 & 13,79 \\
\hline & 13 & 3 & 23,08 \\
\hline & 24 & 2 & 8,33 \\
\hline & 27 & 4 & 14,81 \\
\hline & 27 & 9 & 33,33 \\
\hline & 21 & 3 & 14,29 \\
\hline & 35 & 9 & 25,71 \\
\hline
\end{tabular}


Tab. A XIX: Fremdbefruchtung innerhalb einer Parzelle 2004 und 2005

\begin{tabular}{lccc}
\hline Jahr & $\begin{array}{c}\text { Anzahl } \\
\text { Nachkommen }\end{array}$ & $\begin{array}{c}\text { bestachelte } \\
\text { Nachkommen }\end{array}$ & $\begin{array}{c}\text { Fremdbefruchtung } \\
\text { (\%) }\end{array}$ \\
\hline 2004 & & & \\
& 34 & 20 & 62,98 \\
& 40 & 24 & 64,24 \\
& 43 & 16 & 39,84 \\
& 40 & 26 & 69,59 \\
& 42 & 21 & 53,53 \\
& 43 & 35 & 87,15 \\
& 25 & 14 & 59,96 \\
& 36 & 18 & 53,53 \\
& 38 & 22 & 61,99 \\
& 31 & 23 & 79,44 \\
& & & \\
& 75 & 17 & 26,10 \\
& 57 & 22 & 43,74 \\
& 70 & 18 & 28,89 \\
& 95 & 29 & 35,24 \\
& 85 & 23 & 29,79 \\
& 77 & 27 & 40,95 \\
& 51 & 8 & 17,42 \\
& 57 & 13 & 25,28 \\
79 & 19 & 26,72 \\
& 82 & 19 & 26,21 \\
\hline
\end{tabular}




\section{Danksagung}

Herrn Prof. Dr. Heiko Becker danke ich für die Überlassung des Themas sowie die Diskussionsbereitschaft und wissenschaftliche Betreuung bei der Anfertigung dieser Arbeit.

Besonderer Dank gilt Frau Dr. Sabine von Witzke-Ehbrecht für ihre Betreuung, ihre tatkräftige Unterstützung und die vielen Anregungen in den drei Jahren.

Frau Prof. Dr. Elke Pawelzik danke ich für die Übernahme des Korreferats.

Bei allen Mitarbeiterinnen und Mitarbeitern der Abteilungen Pflanzenzüchtung und Pflanzenbau des Departments für Nutzpflanzenwissenschaften bedanke ich mich für die gute Zusammenarbeit, die Hilfsbereitschaft und die sehr nette Arbeitsatmosphäre. Besonders erwähnen möchte ich hier Britta Apelt, Andreas Henn und Uwe Ammermann für die tolle Unterstützung bei der Durchführung meiner Versuche.

Herrn Dr. Carsten Reinbrecht möchte ich für die Überlassung der Kreuzungen und für die wertvollen Tipps danken.

Mein Dank gilt auch den Mitarbeiterinnen und Mitarbeitern der Universität Hohenheim, des Vereins zur Erhaltung und Rekultivierung von Nutzpflanzen in Brandenburg e.V. und der Getreidezüchtungsforschung Darzau für die Betreuung meiner Versuche an den verschiedenen Standorten. Besonders erwähnen möchte ich Martina Schnieder, Rudolf Vögel und Dr. Karl-Josef Müller.

Diese Arbeit wurde im Rahmen des Bundesprogramms Ökologischer Landbau (BÖL) von dem Bundesministeriums für Ernährung, Landwirtschaft und Verbraucherschutz (BMELV) finanziert. 


\section{Lebenslauf}

\section{Persönliche Daten}

Name:

Sabine Rudolphi

Geburtsdatum:

02.10.1979

Geburtsort:

Paderborn

Familienstand:

ledig

Nationalität:

deutsch

\section{Schulausbildung}

$1986-1990$

Katholische Grundschule, Paderborn-Marienloh

$1990-1999$

Städtisches Gymnasium Theodorianum, Paderborn

\section{Studium}

Okt. 1999 - Feb. 2004 Studium der Agrarwissenschaften an der GeorgAugust-Universität Göttingen

Abschluss: Master of Science

\section{Berufstätigkeit}

seit März 2004

Wissenschaftliche Mitarbeiterin am Department für Nutzpflanzenwissenschaften der Georg-AugustUniversität Göttingen

Abteilung: Pflanzenzüchtung

Projekt des Bundesprogramms Ökologischer Landbau (03OE628):

„Saflor als neue Ölpflanze für den Ökologischen Landbau. Zuchtmethodische Grundlagen und Entwicklung von Schnellmethoden zur Qualitätsbestimmung“" 